\title{
Code of Practice for the Absorbed Dose Determination in High Energy Photon and Electron Beams
}

\section{NEDERLANDSE COMMISSIE VOOR STRALINGSDOSIMETRIE}

Report 18 of the Netherlands Commission on Radiation Dosimetry

Revised edition, August 2012 


\section{Disclaimer regarding NCS reports}

The NCS frequently publishes reports for fellow professionals in which recommendations are given for various quality control procedures or otherwise. The members of the NCS board and the members of the concerning subcommittee do not claim any authority exceeding that of their professional expertise. Responsibility on how the NCS recommendations are implemented lies with the user, taking into account the practice in his/her institution. 


\section{Preface}

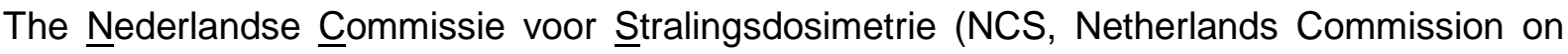
Radiation Dosimetry, http://ncs-dos.org) was officially established on 3 September 1982 with the aim of promoting the appropriate use of dosimetry of ionising radiation both for scientific research and practical applications. The NCS is chaired by a board of scientists, installed upon the suggestion of the supporting societies, including the Nederlandse Vereniging voor Radiotherapie en Oncologie (Netherlands Society for Radiotherapy and Oncology), the Nederlandse Vereniging voor Nucleaire Geneeskunde (Dutch Society of Nuclear Medicine), the Nederlandse Vereniging voor Klinische Fysica (Dutch Society for Medical Physics), the Nederlandse Vereniging voor Radiobiologie (Netherlands Radiobiological Society), the Nederlandse Vereniging voor Stralingshygiëne (Netherlands Society for Radiological Protection), the Nederlandse Vereniging voor Medische Beeldvorming en Radiotherapie (Dutch Society for Medical Imaging and Radiotherapy), the Nederlandse Vereniging voor Radiologie (Radiological Society of the Netherlands) and the Belgische Vereniging voor Ziekenhuisfysici/Société Belge des Physiciens des Hôpitaux (Belgian Hospital Physicists Association).

To pursue its aims, the NCS accomplishes the following tasks: participation in dosimetry standardisation and promotion of dosimetry intercomparisons, drafting of dosimetry protocols, collection and evaluation of physical data related to dosimetry. Furthermore the commission shall maintain or establish links with national and international organisations concerned with ionising radiation and promulgate information on new developments in the field of radiation dosimetry.

Current members of the board of the NCS:

S. Vynckier, chairman B.J.M. Heijmen, vice-chairman E. van Dijk, secretary J. Zoetelief, treasurer A.J.J. Bos J.M.J.Hermans A.A. Lammertsma J.M. Schut F.W. Wittkämper D. Zweers 


\section{Code of Practice for the absorbed dose determination in high energy photon and electron beams \\ Revised edition, August 2012}

This report was prepared by a subcommittee of the Netherlands Commission on Radiation Dosimetry (NCS), consisting of Belgian and Dutch scientists.

Members of the subcommittee:

A.H.L. Aalbers, chairman
M-T. Hoornaert
A. Minken
H. Palmans
M.W.H. Pieksma
L.A. de Prez
N. Reynaert
S. Vynckier

F.W. Wittkämper

NCS, Delft, The Netherlands

For more information on NCS Reports, see http://www.radiationdosimetry.org 
https://doi.org/10.25030/ncs-018 This NCS report has been downloaded on 26 Apr 2023 


\section{User guide}

This report provides recommendations and the methodology for medical physicists to obtain the quantity absorbed dose in water under reference conditions from measurements made with an ionisation chamber in high energy photon and electron beams used in radiotherapy. A prerequisite is the traceable calibration of ionisation chambers in terms of absorbed dose to water in a ${ }^{60} \mathrm{Co}$ reference beam at a standards laboratory.

Chapter 1 gives an introduction on recent developments in dosimetric concepts and methods, measurement standards, etc. resulting in a Code of Practice for clinical photon and electron beams based on the concept of absorbed dose to water. Furthermore it introduces the basic formalism for dose determination and the concept of the beam quality correction factor.

Chapter 2 and 3 contain the Codes of Practice for the dosimetry in respectively high energy photon and electron beams generated by medical linear accelerators.

Appendices are provided giving information on methods, physical and numerical data concerning quantities, correction and conversion factors, on primary absorbed dose standards operated in Belgium and The Netherlands and on the beam quality correction factors to be used in photon and electron dosimetry. Moreover it provides an appendix concerning the differences between this Code of Practice and the previous NCS Codes based on the quantity air kerma. The last appendix deals with the estimation of uncertainty according to international guidelines recommended by ISO and EA. 


\section{Contents}

Preface

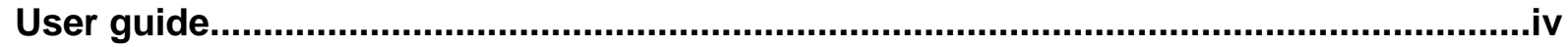

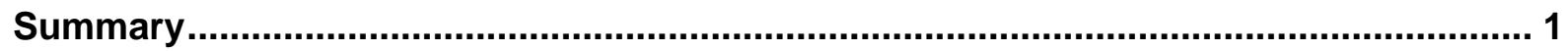

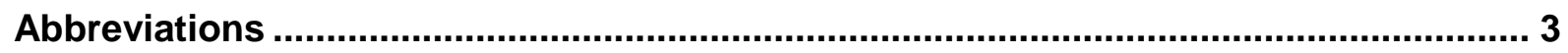

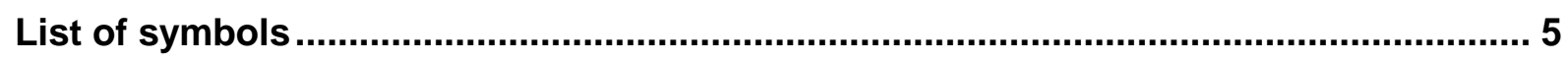

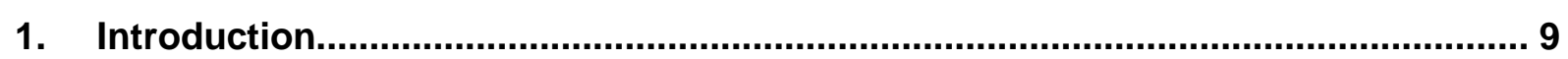

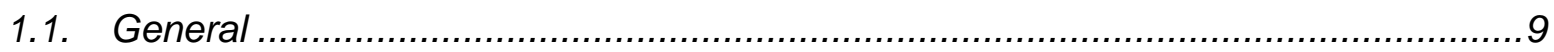

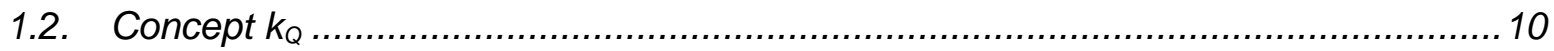

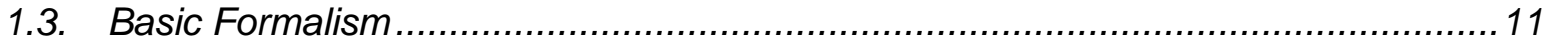

1.4. Available calibration services at LSDG and NMi ........................................... 12

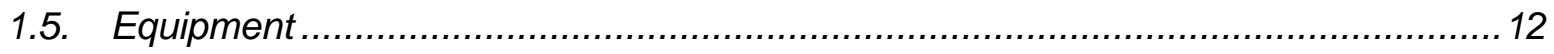

2. Code of Practice for high energy photon beams..................................................16

2.1. General conditions..................................................................................... 16

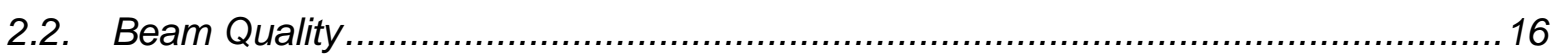

2.3. Determination of $D_{\mathrm{w}, \mathrm{Q}}$ under reference conditions. .......................................... 18

2.4. Determination of $D_{\mathrm{w}, \mathrm{Q}}$ under non-reference conditions ....................................21

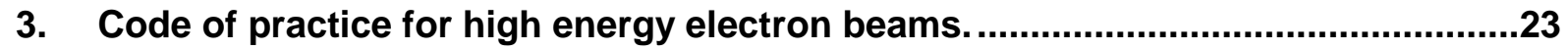

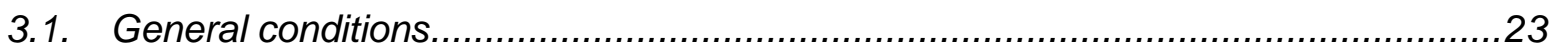

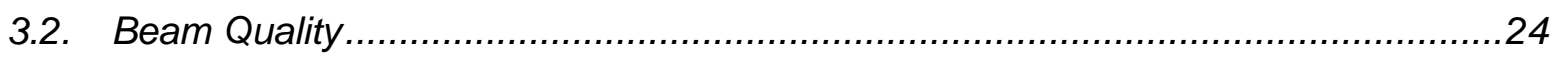

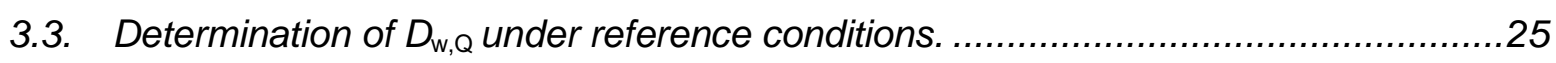

3.4. Determination of $D_{\mathrm{w}, \mathrm{Q}}$ under non-reference conditions ...................................29

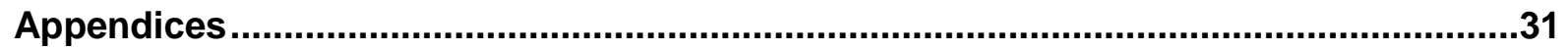

A.1 Instrument data for recommended ionisation chambers....................................31

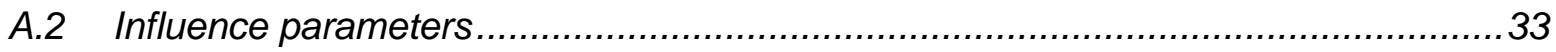

A.3 Absorbed dose to water standards at NMi and LSDG.................................... 38

A.4 Experimental $k_{\mathrm{Q}}$ values and data for clinical high energy photon beams................ 42

A.5 $k_{\mathrm{Q}}$ values and data for clinical high energy electron beams ...............................57

A.6 Expected differences with NCS-2 and NCS-5........................................ 71

A.7 Expression of Measurement Uncertainty ................................................ 78

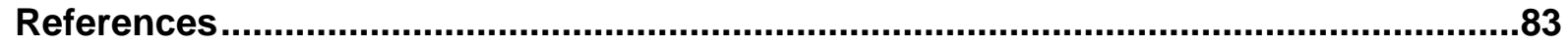

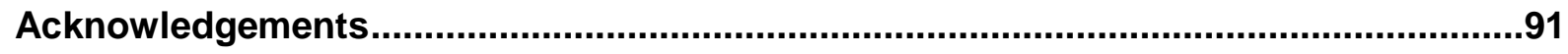


https://doi.org/10.25030/ncs-018 This NCS report has been downloaded on 26 Apr 2023 


\section{Summary}

Absorbed dose to water is the quantity of interest to specify the amount of radiation to be used in radiotherapy and has the advantage that it can be measured more directly than the quantity air kerma. Advances in radiation dosimetry concepts and the development of primary measurement standards based on absorbed dose to water over the last decades offer the possibility to calibrate ionisation chambers directly in terms of absorbed dose to water. Absorbed dose standards have an uncertainty of less than 1\% (1 sd) in photon beams up to $25 \mathrm{MV}$, resulting in improved accuracy in clinical reference dosimetry. Nowadays several international and national Codes of Practice (CoP) for external beam radiotherapy based on absorbed dose standards have been published and adopted in many countries worldwide.

In this report a Code of Practice is presented for the dosimetry in high energy photon and electron beams based on absorbed dose to water standards for ${ }^{60} \mathrm{Co}$ reference beams. The CoP has been written by a Subcommittee of the Netherlands Commission on Radiation Dosimetry (NCS) and corresponds to the current clinical practice in Belgium and The Netherlands. The CoP provides the concepts and methods to determine the absorbed dose in high energy photon and electron beams produced by medical linear accelerators. The CoP covers the reference dosimetry in static, open photon beams with nominal energies between 1 and $25 \mathrm{MV}$ and in static, open electron beams with nominal energies between 4 and $25 \mathrm{MeV}$ produced by conventional linear accelerators. Treatment modalities as intensity modulated therapy (IMRT), stereotactic radiotherapy, robotic radiotherapy, helical tomotherapy, etc. are beyond the scope of this report.

Important features are the simple basic concepts to determine absorbed dose to water under reference conditions in a water phantom and the introduction of a single, chamber dependent correction factor taking into account all effects dependent of the radiation beam quality. For photon beams these beam quality correction factors are based on experimental data, partly measured in selected clinical accelerator beams in Belgium and The Netherlands using a portable water calorimeter.

The present CoP recommends a limited number of ionisation chambers for reference dosimetry in radiotherapy, but the physical concepts outlined in the Code represent a major simplification compared to the previous Codes based on the concept of air kerma employing Bragg-Gray or Spencer-Attix cavity theory. This CoP uses updated information compared to the codes presented in TRS-398 and AAPM TG-51. 
This Code of Practice replaces the protocols based on the concept of air kerma calibration coefficients described in NCS reports 2 and 5. 


\section{Abbreviations}

\begin{tabular}{|c|c|}
\hline AAPM & American Association of Physicists in Medicine \\
\hline BIPM & Bureau International des Poids et Mesures \\
\hline CCEMRI $(I)$ & $\begin{array}{l}\text { Comité Consultatif pour les Etalons de Mesure des Rayonnements } \\
\text { lonisants (Section 1) } \\
\text { (Consultative Committee for Standards of lonising Radiation) } \\
\text { Since September 1997, the CCEMRI and the sections have been } \\
\text { renamed CCRI }\end{array}$ \\
\hline $\operatorname{CCRI}(\mathrm{I})$ & $\begin{array}{l}\text { Comité Consultatif des Rayonnements lonisants (Section 1) } \\
\text { (Consultative Committee for lonising Radiation) }\end{array}$ \\
\hline $\mathrm{CoP}$ & Code of Practice \\
\hline DIN & Deutsches Institut für Normung (German Institute for Standardisation) \\
\hline EA & European Co-operation for Accreditation \\
\hline ICRU & International Commission on Radiation Units and Measurements \\
\hline IAEA & International Atomic Energy Agency \\
\hline TRS-398 & $\begin{array}{l}\text { Absorbed Dose Determination in External Beam Radiotherapy: An } \\
\text { International Code of Practice for Dosimetry Based on Standards of } \\
\text { Absorbed Dose to Water, IAEA, Technical Report Series No. } \\
\text { 398,Vienna, } 2000\end{array}$ \\
\hline LNHB & Laboratoire National Henri Becquerel, France \\
\hline ISO & International Organization for Standardization \\
\hline LSDG & $\begin{array}{l}\text { Laboratorium voor Standaarddosimetrie van de universiteit Gent } \\
\text { (Laboratory for Standard Dosimetry Ghent) }\end{array}$ \\
\hline NCS & $\begin{array}{l}\text { Nederlandse Commissie voor Stralingsdosimetrie (Netherlands } \\
\text { Commission on Radiation Dosimetry) }\end{array}$ \\
\hline NCS-2 & $\begin{array}{l}\text { Nederlandse Commissie voor Stralingsdosimetrie: Code of Practice for } \\
\text { the Dosimetry of High energy Photon Beams, NCS Report 2, } \\
\text { December } 1986\end{array}$ \\
\hline NCS-5 & $\begin{array}{l}\text { Nederlandse Commissie voor Stralingsdosimetrie: Code of Practice for } \\
\text { the Dosimetry of High energy Electron Beams, NCS Report 5, } \\
\text { December } 1989\end{array}$ \\
\hline $\mathrm{NMi}$ & Nederlands Meetinstituut \\
\hline$P L$ & National Physical Laboratory, United Kingdom \\
\hline
\end{tabular}


PMMA

PSDL

TG-51
Polymethyl methacrylate

Primary Standard Dosimetry Laboratory

AAPMs TG-51 protocol for clinical reference dosimetry of high energy photon and electron beams, Med. Phys. 26 1847-1870, 1999 


\section{List of symbols}

In most cases the nomenclature used in publication IAEA TRS398 has been followed.

$D_{w, Q} \quad$ Absorbed dose to water in the beam quality $Q$.

$k_{\text {elec }} \quad$ Calibration coefficient of the electrometer, converts electrometer reading to absolute charge.

$k_{h} \quad$ Factor to correct for the response of an ionisation chamber for the effect of humidity if the calibration coefficient of the chamber is referred to dry air.

$k_{Q, Q_{0}} \quad$ Beam quality correction factor which corrects for the difference in the absorbed dose to water calibration coefficient of a reference beam quality $Q_{0}$ to that of a radiation beam of quality $Q$.

$k_{Q} \quad$ Beam quality correction factor which corrects for the difference in the absorbed dose to water calibration coefficient of $a{ }^{60} \mathrm{Co}$ beam to that of $\mathrm{a}$ radiation beam of quality $Q$. The subscript $Q_{0}$ is omitted when the reference beam quality is ${ }^{60} \mathrm{Co}$.

$k_{\text {pol }} \quad$ Factor to correct for polarity effects affecting the response of the ionisation chamber.

$k_{s} \quad$ Factor to correct for the response of an ionisation chamber for the lack of complete charge collection.

$k_{T P} \quad$ Factor to correct for the response of an ionisation chamber for deviations of temperature and pressure from normal conditions.

$M_{\text {corr, } Q} \quad$ The electrometer reading corrected for any difference between the ambient air conditions affecting the ionisation chamber at the time of measurement and the standard ambient air conditions for which the calibration coefficient applied (air temperature, pressure and humidity), for ion recombination and for polarity effects. 
$M_{Q} \quad$ The uncorrected reading of the instrument.

$N_{D, w} \quad$ The absorbed dose to water calibration coefficient for an ionisation chamber (or for the dosimeter assembly: ionisation chamber and electrometer) at the reference beam quality ${ }^{60} \mathrm{Co}$.

$N_{D, w, Q} \quad$ The absorbed dose to water calibration coefficient for an ionisation chamber (or for the dosimeter assembly: ionisation chamber and electrometer) at the beam quality $Q$.

$N_{D, \mathrm{w}, \text { Qcross }} \quad$ The absorbed dose to water calibration coefficient for an ionisation chamber (or for the dosimeter assembly: ionisation chamber and electrometer) at the cross calibration quality $Q_{\text {cross }}$.

$\% d d(10)_{\mathrm{x}} \quad$ The photon component of the percentage depth dose at $10 \mathrm{~cm}$ depth in a $10 \mathrm{~cm} \times 10 \mathrm{~cm}$ field on the surface of a water phantom at an SSD of $100 \mathrm{~cm}$, used as a beam quality index in photon beams.

$p_{\text {cav }} \quad$ Factor to correct for the response of an ionisation chamber for deviations from Bragg-Gray conditions due to the effect of the air cavity in the medium.

$p_{\text {cel }} \quad$ Corrects for the presence of the central electrode.

$p_{\text {dis }} \quad$ Corrects for the difference in ionisation at the effective point of measurement and the depth at which the absorbed dose is stated.

$p_{\text {eff }}$ is the effective point of measurement of an ionisation chamber. For a radiation beam incident to the chamber from one direction the effective point of measurement is shifted from the position of chamber centre towards the source by a distance depending on the beam quality and the chamber type. For plane-parallel chambers the effective point of measurement is assumed at the inner surface of the entrance window.

$p_{\text {wall }} \quad$ Corrects for the difference in composition between the ionisation chamber wall and water. 
$Q$

$Q_{0}$

$\operatorname{rdg}$

$R_{50, \text { dos }}$

$R_{50 \text {,ion }}$

$R_{p}$

$r_{\mathrm{cyl}}$

sd

SCD

SDD

SSD

$\mathbf{s}_{m, \text { air }}$

$\mathrm{TPR}_{20,10}$

$u_{i}$
Symbol to indicate the quality of a radiation beam.

Symbol to indicate the reference beam quality used for calibration of an ionisation chamber (usually in a Standards Laboratory).

Reading of a dosimeter in arbitrary units.

Half-value depth of dose in water in $\mathrm{cm}$, used as the beam quality index for electron beams.

Half-value depth of ionisation in water in $\mathrm{cm}$.

Practical range in $\mathrm{cm}$ for electron beams.

Cavity radius of a cylindrical ionisation chamber.

Standard deviation.

Source-chamber distance.

Source-detector distance.

Source-surface distance.

Stopping-power ratio medium to air, defined as the ratio of the mean restricted mass stopping powers of materials $\mathrm{m}$ and air, averaged over an electron spectrum.

Tissue-phantom ratio in water at depths of 20 and $10 \mathrm{~cm}$, for a field size of $10 \mathrm{~cm} \times 10 \mathrm{~cm}$ and a SCD of $100 \mathrm{~cm}$, used as the beam quality index for high energy photon radiation.

Standard uncertainty of a quantity. 
Combined standard uncertainty of a quantity.

$U$

Polarising voltage applied to the ionisation chamber.

$W_{\text {air }} \quad$ The mean energy expended in air per ion pair formed.

$Z_{\max } \quad$ Depth of maximum dose in $(\mathrm{cm})$.

$z_{\text {ref }}$

Reference depth in $(\mathrm{cm})$ for in-phantom measurements. 


\section{Introduction}

\subsection{General}

Until recently, it has been generally recommended to perform reference dosimetry of clinical high energy $\mathrm{x}$-ray and electron beams with ionisation chambers calibrated in terms of air kerma. To this end, the NCS has published NCS report 2 [1] and NCS report 5 [2] containing Codes of Practice for the dosimetry of high energy photon beams and electron beams, respectively, based on air kerma standards. These CoP's, issued by the NCS, were based on a number of simple concepts. A strictly limited number of ionisation chambers, routinely used in the Netherlands and Belgium were selected as reference dosimeters. In addition, the concept of a single conversion factor $C_{\mathrm{w}, \mathrm{u}}$ or $C_{\mathrm{w}, \mathrm{e}}$ for each reference chamber, as function of the beam quality index (i.e. a tissue phantom ratio, $\operatorname{TPR}_{20,10}$, for photon beams and the mean energy at the phantom surface, $\bar{E}_{0}$, for electron beams) was applied. These recommendations were compared by Mijnheer and Wittkämper [3] with similar international recommendations of the AAPM TG-21 [4] and IAEA TRS-277 [5]. The recommendations of the NCS are applied in every radiotherapy institute in the Netherlands and Belgium since 1986. Their implementation was audited on a regular basis $[6,7,11]$ in most of the radiotherapy centres of both countries.

In the last two decades, reference dosimetry based on absorbed dose calibration coefficients has gained much attention. Advances in radiation dosimetry concepts and the development of absorbed dose to water standards by Primary Standard Laboratories have reduced the uncertainty in absorbed dose determination in external beam therapy. New codes of practice for reference dosimetry in clinical high energy photon and electron beams, based on absorbed dose to water standards, have been published recently. They are to replace the air kerma based codes of practice applied for the past twenty years.

Task Group 51 of the AAPM developed a protocol for dosimetry of high energy photon and electron beams [9]. The IAEA published a protocol for dosimetry of all external radiotherapy beams except neutron beams: TRS-398 [10].

Since the publication of these protocols a number of studies have already been devoted to comparing the two dosimetry formalisms with each other, or with air kerma based formalisms [12-15]. In general they discuss results for a limited number of chambers and and often for one or two chamber types in each study. In a recent work, Palmans et al.[16] compared within an experimental study the absorbed dose to water based dosimetry versus air kerma 
based dosimetry for high energy photon beams. A set of nine ionisation chambers was calibrated both in terms of air kerma and in terms of absorbed dose to water in ${ }^{60} \mathrm{Co}$. Graphite-walled ionisation chamber types that are recommended for reference dosimetry in recent formalisms were selected. Their objective was to determine what consistency could be expected using the new formalisms and what differences could be expected compared to air kerma based formalisms (i.e. NCS-2). In another study [17] absorbed dose to water based dosimetry was compared with air kerma based dosimetry (NCS-5) in high energy electron beams. These measurements should be seen as preparatory for the present NCS report.

The NCS subcommittee on "Uniformity Dosimetry Protocols" has written a CoP based on the absorbed dose to water concept covering reference dosimetry in clinical high energy photon beams with nominal acceleration potentials between 1 and $25 \mathrm{MV}$ and high energy electron beams with nominal energies between 4 and $25 \mathrm{MeV}$. The present CoP introduces a single beam quality correction factor taking into account all effects dependent of the radiation beam quality. For photon beams the beam quality correction factors are based on experimental data, partly measured in selected clinical accelerator beams in Belgium and the Netherlands, using a portable water calorimeter. For electron beams theoretically calculated correction factors are given, based on recent literature data. Furthermore, the CoP recommends a limited number of ionisation chambers for reference dosimetry in radiotherapy and uses updated information compared to the codes presented in TRS-398 and TG-51.

This CoP will replace the codes described in NCS reports 2 and 5. The guidelines and recommendations for clinical reference dosimetry in photon and electron beams are discussed in chapters 2 and 3 of this document.

\subsection{Concept $k_{Q}$}

Hohlfeld [18] proposed a practical concept for high energy photon dosimetry in Germany. This concept was based on one absorbed dose to water calibration coefficient in a ${ }^{60} \mathrm{Co}$ beam and a beam quality correction factor $k_{Q, Q_{0}}$, accounting for the high energy photon beam quality $Q$ and has further been developed by Andreo [19] and Rogers [20]. The beam quality correction factor is defined as the ratio of absorbed dose to water calibration coefficients in the clinical beam quality $Q, N_{D, w, Q}$ and the reference (calibration) beam quality $Q_{0}, N_{D, w, Q}$ : 


$$
k_{Q, Q_{0}}=\frac{N_{D, w, Q}}{N_{D, w, Q_{0}}}
$$

The reference beam quality $Q_{0}$, used for calibration of ionisation chambers, is ${ }^{60} \mathrm{Co}$ gamma radiation. The beam quality correction factor has been measured directly for selected reference ionisation chambers in various clinical high energy photon beams. The measurements involved a portable water calorimeter developed by NMi to determine the absorbed dose to water in the clinical photon beam. The results of this experimental work are described in appendix A.4. These measurements together with other experimental data from the literature form the basis of the present CoP for high energy photon beams of the NCS. For photon beams these data are presented as a function of $\mathrm{TPR}_{20,10}$. For electron beams, theoretically calculated correction factors, based on recent literature data, are given as a function of the half-value depth in water $R_{50, \text { dos }}$.

\subsection{Basic Formalism}

The absorbed dose to water at the reference depth in water for a user beam $Q$ in absence of the ionisation chamber is given by:

$$
D_{w, Q}=M_{c o r r, Q} N_{D, w, Q_{0}} k_{Q, Q_{0}}
$$

where:

$M_{c o r r, Q}$ reading of the electrometer corrected to ambient reference conditions and for the effects of recombination, polarity and the influence of the electrometer,

$N_{D, w, Q_{0}}$ the calibration coefficient for absorbed dose to water for a reference photon beam quality $Q_{0}$,

$k_{Q, Q_{0}} \quad$ accounts for the effects of the differences between the beam quality $Q$ and the reference beam quality $Q_{0}$.

The $k_{Q, Q_{0}}$ factors listed for high energy photon and electron beams are all related to the reference beam quality of ${ }^{60} \mathrm{Co}$ gamma radiation. Therefore the symbol $Q_{0}$ in $k_{Q, Q_{0}}$ will be omitted in the following sections, yielding $k_{Q}$. 


\subsection{Available calibration services at LSDG and NMi}

LSDG and NMi operate calibration services for absorbed dose to water in ${ }^{60} \mathrm{Co}$ gamma radiation. The primary standards involved are based on sealed water calorimeters according to a design of Domen [21]. The dose response of the calorimeter in a water phantom is transferred to a set of transfer ionisation chambers. LSDG uses three NE 2571 chambers as transfer instruments, whereas NMi uses three NE 2611A chambers for the determination of the long-term stability of the absorbed dose to water value. Secondary (local) standards including plane-parallel ionisation chambers from hospitals or other users are directly calibrated against the primary standards for absorbed to water in both laboratories, ensuring a short traceability chain. Further details on the construction and performance characteristics of the LSDG and NMi water calorimeters are given in Appendix A.3. It should be noted that the ionisation chambers employed as secondary (local) standard should fulfil the requirements outlined in this CoP.

\subsection{Equipment}

1. This CoP is based on the use of ionisation chambers for reference dosimetry in clinical photon and electron beams. A typical dosimeter system consists of an ionisation chamber, an electrometer and a radioactive check source. The ionisation chambers should preferably be designed for absorbed dose measurements in water and the construction should be as homogeneous and water equivalent as possible. Cylindrical ionisation chambers may be used for measurements in ${ }^{60} \mathrm{Co}$ gamma radiation, photon beams and electron beams with energy above approximately $10 \mathrm{MeV}$. The chamber should have a volume between about 0.1 and $1 \mathrm{~cm}^{3}$. The air cavity of the chamber should not be sealed, but designed to reach rapidly equilibrium conditions with the ambient temperature and air pressure.

2. Only graphite-walled ionisation chambers are recommended for reference dosimetry in this CoP. In general, these chambers have a more uniform response and better long-term stability than plastic walled chambers. However, some plastic-walled chambers have been demonstrated to show a stable response over time. Plastic-walled chambers are more robust and therefore often preferred as field instruments to perform routine measurements. The characteristics and physical data of the graphite-walled ionisation chambers most commonly 
employed as local standards for reference dosimetry and recommended as reference instruments in this CoP are given in Appendix A.1.

3. Plane-parallel chambers are recommended for the use in electron beams. The use of plane-parallel chambers as reference instrument below $10 \mathrm{MeV}$ is mandatory, because the uncertainty in the perturbation correction for a Farmer type cylindrical chamber becomes significant at low electron beam energies. Wellguarded plane-parallel chambers have a small perturbation effect, which will be further discussed in Appendix A.5. The chamber samples the electron fluence through the front window, so that the contribution of electrons entering the chamber through the side walls is minimized. The effective point of measurement, $p_{\text {eff }}$, is taken at the centre on the inner surface of the entrance window of the plane-parallel chamber for all electron beam energies and depths. Therefore, it is convenient to choose this as the reference point of the chamber during measurements. Further details and physical data for plane-parallel chambers recommended in this CoP can be found in Appendix A.5.

4. Water is recommended as phantom material, the use of plastic phantoms is not allowed. The phantom should be a full scatter phantom extending at least $5 \mathrm{~cm}$ outside the beam edges and at least $10 \mathrm{~cm}$ beyond the centre of the ionisation chamber along the beam axis. The dimensions of the water phantom should be at least $30 \mathrm{~cm} \times 30 \mathrm{~cm} \times 30 \mathrm{~cm}$. The beam direction is vertical, perpendicular to the water surface. The water phantom must be open in this direction. These are the reference conditions for this CoP. The use of (water equivalent) plastics as phantom material $[23,24]$ is not allowed for reference dosimetry, because in general they give rise to the largest discrepancies in the determination of absorbed dose to water in clinical photon and electron beams [25].

5. For non-reference conditions, where the beam direction is horizontal, the window of the phantom, usually made of plastic, should have a thickness $t_{\text {win }}$ between 2 and $5 \mathrm{~mm}$. The water equivalent thickness of the phantom, expressed in $\mathrm{g} / \mathrm{cm}^{2}$, has to be taken into account when the position of the chamber is determined in the phantom. The equivalent thickness $\left(\right.$ in $\mathrm{g} / \mathrm{cm}^{2}$ ) is calculated by multiplying $t_{\text {win }}$ by the mass density $\rho_{\mathrm{pl}}$ of the plastic (in $\mathrm{g} / \mathrm{cm}^{3}$ ). For plastics commonly used, like PMMA and clear polystyrene, nominal mass densities of $\rho_{\mathrm{PMMA}}=1.19\left(\mathrm{~g} / \mathrm{cm}^{3}\right)$ and $\rho_{\text {polystyrene }}=1.06\left(\mathrm{~g} / \mathrm{cm}^{3}\right)$ may be used to calculate the water equivalent thickness of the phantom window [22]. Note, that differences in the density of water equivalent plastics and polystyrene between manufacturers may exist [25]. 
6. If an ionisation chamber is not designed for direct use in a water phantom, it must be used with a close-fitting waterproof sleeve, made of low-Z materials (e.g. PMMA). The wall of the sleeve should be sufficiently thin $(<1.0 \mathrm{~mm})$ to allow the chamber to achieve thermal equilibrium with the phantom in typically 2 to 3 minutes per degree of temperature difference. The sleeve should be vented to allow the air pressure to reach ambient air pressure rapidly; an air gap of 0.1 $0.3 \mathrm{~mm}$ between the sleeve and the chamber is sufficient. Note that the waterproof sleeve should not be left in the water longer than necessary to perform the measurement, in order to minimize the build up of water vapour around the ionisation chamber. As the sleeve is to be considered as a component of the ionisation chamber, preferably the same sleeve should be used during calibration of the chamber and measurement assembly at LSDG or NMi.

7. The measuring assembly for the measurement of current (or charge) includes an electrometer with a good long-term stability (variation less than $\pm 0.5 \%$ over one year) and a power supply for the polarizing voltage of the ionisation chamber. The electrometer and the ionisation chamber may be calibrated separately. This is particularly useful in centres that have several electrometers and/or chambers.

8. Sufficient time should be allowed for the dosimeter to reach thermal equilibrium. Some mains powered electrometers are best switched on for at least $2 \mathrm{~h}$ before use to allow stabilization. It is always advisable to pre-irradiate an ionisation chamber with $2-5$ Gy to achieve charge equilibrium in the different materials. It is especially important to operate the measuring system under stable conditions whenever the polarity or polarizing voltage are modified which, depending on the chamber and sometimes on the polarity, might require several (up to 20) minutes.

9. The leakage current should always be measured before and after irradiation, and should be small compared with the current obtained during the irradiation (less than approximately $0.1 \%$ of the measurement current and normally of the same sign). Chambers with a leakage current which is large (approximately larger than $1 \%$ of the measurement current) or variable in time should not be used.

10. Only ionometric measurements are considered in this Code of Practice for reference dosimetry. We describe the calibration of field instruments (ionisation chamber and electrometer combination). The same approach can be used when various ionisation and electrometer combinations are used in clinical practice. It is left to the user to make a protocol designed for the users practice. 
11. For relative measurements with ionisation chambers the displacement of the effective point of measurement has to be corrected for. For photons this is included in the calibration coefficient in the reference situation.

12. The use of other dosimetry detectors is not within the scope of this report, although for relative measurements other detectors may be more suitable. Care must be taken to use other detectors in relative dosimetry and to apply proper correction factors in non-reference situations.

13. Although not allowed for use in reference dosimetry, plastic phantoms can be used for routine quality assurance measurements, provided the relationship between dosimeter readings in plastic and water has been established for the user beam at the time of calibration. This will involve a careful comparison with measurements in water, which should be performed prior to the routine use of the phantom, and periodic checks at reasonable intervals might be also needed to assure the validity and consistency of the original comparison result. 


\section{Code of Practice for high energy photon beams}

\subsection{General conditions}

The present CoP for high energy photon beams of the NCS will follow the concept below:

1. The CoP is based on recommendations for a limited number of graphite-walled, cylindrical ionisation chambers. These are the same models as those used in the water calorimetry campaign and are listed in Table 1 (see also Appendix A.1). The recommendations regarding measurement equipment given in section 1.5 should be followed.

2. The CoP will only recommend formalisms for high energy photon beams based on a ${ }^{60} \mathrm{Co}$ calibration. The symbol used for the beam quality correction factor will be $k_{Q}$.

3. The beam quality correction factors $k_{Q}$ are based on the methods described in Appendix A.4.

4. The values of $k_{Q}$ obtained from the fit in Appendix A.4 are valid for $\operatorname{TPR}_{20,10}$ values ranging from 0.62 to 0.82 ; this corresponds to a nominal photon energy range from about $6 \mathrm{MV}$ to $25 \mathrm{MV}$. The proposed fit to the $k_{Q}$ data can also be applied to lower energies without loss of accuracy. However at higher energies extrapolation of the data will increase the uncertainty of $k_{Q}$.

\subsection{Beam Quality}

For high energy photons produced by clinical accelerators the beam quality $Q$ is specified by the tissue-phantom ratio, $\mathrm{TPR}_{20,10}$ [26-29]. This parameter is defined as the ratio of the absorbed doses at depths of $20 \mathrm{~cm}$ and $10 \mathrm{~cm}^{*}$ in a water phantom, measured with a constant source-chamber distance ${ }^{\dagger}$ of $100 \mathrm{~cm}$ and a field size of $10 \mathrm{~cm} \times 10 \mathrm{~cm}$ at the plane of the chamber as can be seen from Figure 1. The choice of $T P R_{20,10}$ is justified in Appendix A.4 where an analysis is made of various $k_{Q}$ factors as function of $\operatorname{TPR}_{20,10}$ and $\% d d(10)_{\times}[9]$. The experimental set up for measuring $\operatorname{TPR}_{20,10}$ is shown in Figure 1. This parameter is obtained as the ratio of the absorbed doses at depths of $20 \mathrm{~cm}$ and $10 \mathrm{~cm}$ under following reference conditions:

\footnotetext{
* For simplicity the depth in water is expressed in $\mathrm{cm}$ instead of $\mathrm{g} / \mathrm{cm}^{2}$, this will lead to differences in the evaluation of TPR 20,10 amounting to $0.1 \%$ at maximum.

${ }^{+} \mathrm{TPR}_{20,10}$ is normally measured at a fixed SCD (source-chamber distance). This quantity is however independent of the distance of measurement between $S C D=80 \mathrm{~cm}$ and $S C D=150 \mathrm{~cm}$ for a field size of $10 \mathrm{~cm} \times 10 \mathrm{~cm}$ at the detector plane [1].
} 
- water phantom,

- constant SCD (source-chamber distance) of $100 \mathrm{~cm}$,

- field size of $10 \mathrm{~cm} \times 10 \mathrm{~cm}$ at the axis of the chamber.

The recommendations regarding ionisation chambers, phantoms and sleeves, given in Section 1.5 should be followed. Although the definition of $\mathrm{TPR}_{20,10}$ is strictly made in terms of absorbed dose ratios, the use of ionisation ratios provides an acceptable accuracy due to the slow variation with depth of water/air stopping-power ratios and the assumed constancy of perturbation factors beyond the depth of dose maximum. Small variations of the recombination correction factor $k_{s}$, as defined in Appendix A.2, may appear at different depths and consequently this variation should be investigated and taken into account if there is a variation with depth.

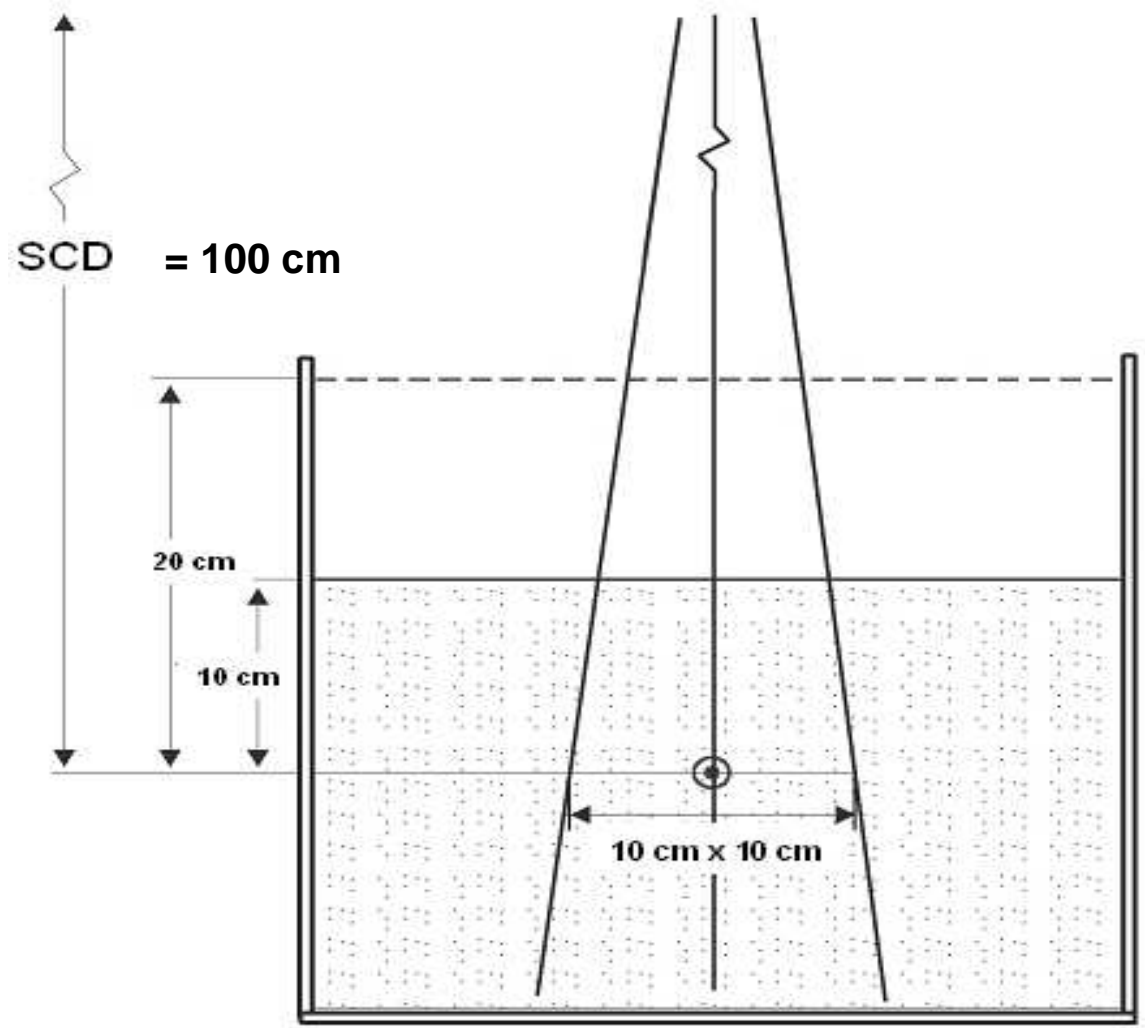

Figure 1. Experimental set-up for the determination of the beam quality index $\operatorname{TPR}_{20,10}$. The source-to-chamber distance (SCD) is kept constant at $100 \mathrm{~cm}$ and measurements are made with $10 \mathrm{~cm}$ and $20 \mathrm{~cm}$ of water over the chamber. The field size at the position of the centre of the chamber, which is placed at the isocentre of the machine, is $10 \mathrm{~cm} \times 10 \mathrm{~cm}$. 


\subsection{Determination of $D_{\mathrm{w}, \mathrm{Q}}$ under reference conditions.}

This section is based upon an absorbed dose to water calibration coefficient $N_{D, w}$ for a dosimeter in a reference ${ }^{60} \mathrm{Co}$ beam. The formalism to derive the absorbed dose to water for photon beams is described in section 1.3. Detailed information on the uncertainties, calculation of the influence quantities and numerical data needed for $k_{Q}$ calculation can be found in the Appendices A.1-A.7. Moreover, these appendices will list the quantities, symbols and definitions, a summary of water calorimetry methodology and expected differences with NCS-2.

The recommendations are stated below:

1. The local standard shall be a graphite-walled, cylindrical ionisation chamber, listed in Table 1.

2. The local standard shall be calibrated in terms of absorbed dose to water, $N_{D, w}$, at a Primary Standard Dosimetry Laboratory (PSDL) ${ }^{*}$ in a ${ }^{60} \mathrm{Co}$ beam under the conditions described in section 1.4.

3. A water phantom shall be used with a vertical beam; the chamber shall be fitted in the water with a thin waterproofing sheath ${ }^{\dagger}$ made of PMMA, not thicker than $1 \mathrm{~mm}$. More information on phantoms and sleeves is given in section 1.5.

4. For the determination of the absorbed dose to water in the users beam, the chamber shall be placed on the beam axis with the geometrical centre of the chamber at the reference depth $z_{\text {ref }}=10 \mathrm{~cm}$.

5. The water surface shall be positioned at the isocentre of the linear accelerator for a fixed source-surface distance $(S S D=100 \mathrm{~cm}$ ) measurement or the detector shall be positioned at the isocentre of the linear accelerator for a fixed sourcechamber distance $(S C D=100 \mathrm{~cm})$ measurement. A field size of $10 \mathrm{~cm} \times 10 \mathrm{~cm}$ at the isocentre shall be used.

6. For the local standard the absorbed dose to water $D_{w, Q}$ at the reference depth will be given by:

$$
D_{w, Q}=M_{c o r r, Q} N_{D, w} k_{Q}
$$

where:

\footnotetext{
"For the Netherlands the Primary Standard Dosimetry Laboratory (PSDL) is the NMi at Delft whereas for Belgium the Primary Standard Dosimetry Laboratory is the LSDG at Ghent.

${ }^{\dagger}$ All $k_{Q}$ values including those for the waterproof Wellhöfer chamber have been determined using a waterproof sheath $(\leq 1 \mathrm{~mm})$. The user employing the Wellhöfer chamber without the waterproof sheath has to account for the difference in the dose determination, which is expected to be less then $0.2 \%$.
} 
$D_{w, Q}$ is the absorbed dose to water in the users beam $Q$ and in absence of the chamber, at the reference depth $z_{\text {ref }}=10 \mathrm{~cm}$,

$M_{c o r r, Q}$ is the electrometer reading, corrected for influence quantities as described in Appendix A.2,

$N_{D, w}$ is the absorbed dose to water calibration coefficient in the ${ }^{60} \mathrm{Co}$ reference beam quality, given by the Standards Laboratory at reference ambient conditions (normally $T_{\text {ref }}=20^{\circ} \mathrm{C}$ and $P_{\text {ref }}=$ $101.325 \mathrm{kPa}$ and $50 \%$ relative humidity),

$k_{Q} \quad$ is the beam quality correction factor that depends on the chamber type and radiation beam quality of the user beam. For the 4 recommended ionisation chamber types of Table $1 k_{Q}$ values as function of the quality index $\mathrm{TPR}_{20,10}$ are obtained from equation:

$$
k_{Q}=0.80+0.20 \cdot \frac{1+e^{C \cdot\left(0.57-X_{0}\right)}}{1+e^{C \cdot\left(T P R_{20,10}-X_{0}\right)}}
$$

where the parameters $\mathrm{C}$ and $\mathrm{X}_{0}$ of this equation for the recommended ionisation chambers are given in Table 1. The $k_{Q}$ are valid in the photon energy range of $6 \mathrm{MV}$ to $25 \mathrm{MV}$. The data obtained using equation (4) are plotted in Figure 10 (see Appendix A.4, section.A.4.5.2).

7. $k_{Q}$ equals 1.000 for for determination of the absorbed dose to water in $a{ }^{60} \mathrm{Co}$ beam.

8. The electrometer reading $M_{Q}$ should be corrected for all influence quantities:

$$
M_{\text {corr }, Q}=M_{Q} k_{T P} k_{h} k_{p o l} k_{s}
$$

where:

$k_{T P}$ is the correction factor for pressure and temperature in the air cavity at the time of measurement,

$k_{h}$ is the correction for humidity in the air cavity at the time of measurement, 
$k_{\text {pol }}$ is the correction on the chamber reading due to the applied polarity of the polarizing voltage,

$k_{s} \quad$ is the correction for incomplete charge collection in the ionisation chamber due to recombination.

These correction factors have to be determined according to the procedures explained in Appendix A.2.

9. Under these conditions the relative standard uncertainty of the absorbed dose to water $D_{w, Q}$ at the reference depth $z_{\text {ref }}=10 \mathrm{~cm}$ for a high energy photon beam is estimated to be $1.0 \%$ ( $1 \mathrm{sd})$. More details on the estimation of this relative combined standard uncertainty are given in Appendix A.4.

Table 1: List of the recommended graphite-walled cylindrical chambers together with the parameters of the sigmoid fit (see Appendix A.4). Using these parameters in equation 4 allows obtaining the $k_{Q}$ values as a function of the beam quality $\operatorname{TPR}_{20,10}$ for these ionisation chambers.

\begin{tabular}{|c|c|c|}
\hline Chamber type & $X_{0}$ & $C$ \\
\hline $\begin{array}{c}\text { NE 2561/NE } \\
2611\end{array}$ & 0.8971 & 15.15 \\
\hline $\begin{array}{c}\text { NE2571 } \\
\text { PTW30012 } \\
\text { Wellhöfer FC65G }\end{array}$ & 0.9198 & 11.67 \\
\hline
\end{tabular}




\subsection{Determination of $D_{\mathrm{w}, \mathrm{Q}}$ under non-reference conditions}

Determination of $D_{\mathrm{w}, Q}$ under non-reference conditions is not part of the CoP, however a few points should be considered. Field instruments shall be calibrated against the reference instrument at the radiation qualities at which they are to be used. This calibration shall be in terms of absorbed dose to water.

1. Only ionisation chambers are considered in this protocol.

2. Field ionisation chambers shall be calibrated in terms of absorbed dose to water against the local standard, with their central axis at the same reference depth $z_{\text {ref, }}$, SSD and field size as stated in section 2.3. A water phantom shall be used. This calibration has to be done for each beam quality at which they are to be used. The absorbed dose to water calibration coefficient $N_{w, Q}^{\text {field }}$ at the users beam quality $Q$ for the field ionisation chamber is then given by:

$$
N_{w, Q}^{\text {field }}=D_{w, Q}^{s t d} / M_{c o r r, Q}^{\text {field }}
$$

where:

$D_{W, Q}^{s t d}$ is the absorbed dose to water in the users beam at the reference depth $z_{\mathrm{ref}}=10 \mathrm{~cm}$ determined by the local standard,

$M_{c o r r, Q}^{\text {field }}$ is the electrometer reading of the field instrument, corrected for influence quantities as described in Appendix A.2.

Preferably an additional monitor chamber in the radiation field should be used to normalise the reading in order to account for beam fluctuations.

3. If the field instrument is one of the recommended ionisation chambers from Table 1 , than this calibration can be considered as a cross calibration. The obtained calibration coefficient $N_{w, Q}^{\text {field }}$ is applicable for all photon beams by calculating a ${ }^{\text {"60} C o ~ c a l i b r a t i o n ~ c o e f f i c i e n t " ~ f o r ~ t h e ~ f i e l d ~ i n s t r u m e n t ~ f r o m ~ i t . ~ T h e ~}{ }^{60} \mathrm{Co}$ related calibration coefficient can be obtained from the ratio $N_{w, Q}^{f i e l d}$ and $k_{Q}$. This calibration coefficient can subsequently be used according to the CoP.

4. Relative measurements (i.e. determination of the absorbed dose at other points in the phantom than $z_{\text {ref }}$ ) can be performed by different types of ionisation chambers. To this end, the effect of displacement, which implicitly is already taken into account in $k_{Q}$ (for photons) for dosimetry under reference conditions, should be 
taken into account by positioning the effective point of measurement of the ionisation chamber to the depth of measurement. This effective point of measurement is 0.6 times the inner radius in front of the geometrical centre for cylindrical ionisation chambers [5] and on the inner site of the front window for plane parallel ionisation chambers [30].

5. The use of other dosimetry detectors (i.e. diodes, diamond detectors, TLD, Alanine, scintillator detectors, etc.) is not within the scope of this report. 


\section{Code of practice for high energy electron beams.}

\subsection{General conditions}

The present CoP for high energy electron beams of the NCS will follow the concept below:

1. The CoP is based on recommendations for a limited number of graphite-walled, Farmer type and plane-parallel ionisation chambers. The Farmer type cylindrical chamber types are the same models as used in high energy photon beams and recommended only for electron energies with beam quality $R_{50 \text {,dos }}$ larger than $4 \mathrm{~cm}^{*}$ in water. For lower electron energies only plane-parallel chambers are recommended. The recommendations regarding measurement equipment given in section 1.5 should be followed.

2. For dosimetry in a high energy electron beam using a Farmer type cylindrical ionisation chamber (only for $R_{50 \text {,dos }}>4 \mathrm{~cm}$ ) the recommendation is based on a calibration in a ${ }^{60} \mathrm{Co}$ reference beam. Dosimetry using a plane-parallel ionisation chamber (for any beam quality $Q$ ) is strongly recommended to be based on a cross-calibration against a Farmer type cylindrical ionisation chamber in a high energy electron beam. This beam is referred to as the cross calibration beam quality with an $R_{50 \text {,dos }}$ recommended to be larger than $7 \mathrm{~cm}$. This method requires a beam quality correction factor $k_{Q, Q c r o s s}$, which converts the calibration coefficient of the plane-parallel ionisation chamber in the cross-calibration beam quality to the beam quality $Q$. Only for the case this option is not available to a particular user, the protocol also provides the data necessary to perform dosimetry using a plane-parallel ionisation chamber based on a dose to water calibration in a ${ }^{60} \mathrm{Co}$ reference beam.

3. The beam quality correction factors $k_{Q}$ and $k_{Q, Q c r o s s}$ are based on the methods described in appendix A.5 and are given as a parameterised function of the beam

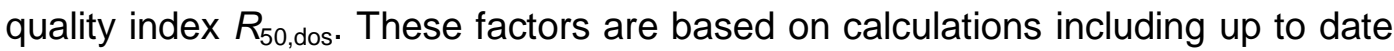
information from the literature.

4. Values of $k_{Q}$ and $k_{Q, Q c r o s s}$ are provided for $R_{50 \text {,dos }}$ values ranging from $1 \mathrm{~cm}$ to $12 \mathrm{~cm}$ (although their validity extends to $20 \mathrm{~cm}$ ).

\footnotetext{
* For simplicity $R_{50, \text { dos }}$ and the depth $z$ in water is expressed in $\mathrm{cm}$ instead of $\mathrm{g} / \mathrm{cm}^{2}$, this leads to differences in the evaluation of $R_{50 \text {,dos }}$ of about $0.2 \%$.
} 


\subsection{Beam Quality}

For high energy electron beams produced by clinical accelerators, the beam quality $Q$ is specified by the $50 \%$ dose level beyond the dose maximum, $R_{50 \text {,dos, }}$ as illustrated in Figure 2 . The parameter $R_{50 \text {,dos }}$ is obtained as the depth beyond the dose maximum where the absorbed dose to water is $50 \%$ of its maximum value. The beam quality index can be determined either directly from a depth dose curve or from a depth ionisation curve. Depth ionisation curves are preferably measured using a plane-parallel ionisation chamber, with the reference point of the chamber taken as the centre of the inner surface of its front window. If a cylindrical chamber would be used, the reference point has to be taken as 0.5 times the inner radius of the chamber in front of the geometrical centre*, as illustrated in Figure 3.

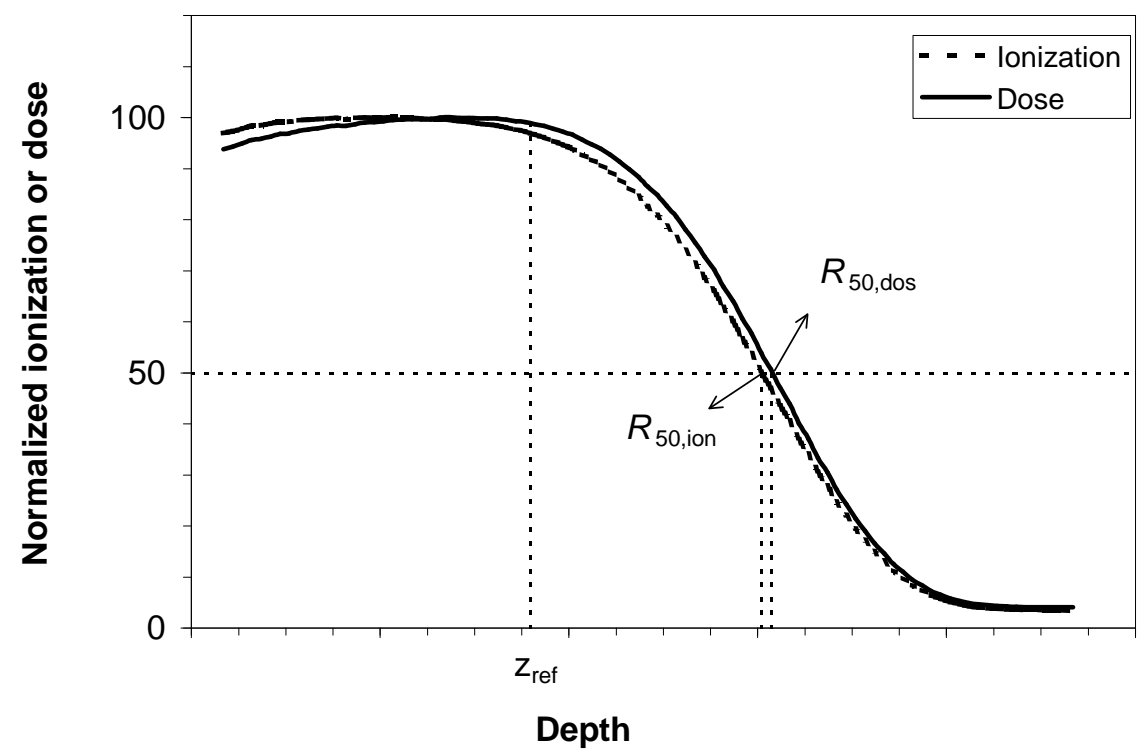

Figure 2: Definition of the beam quality index $R_{50, \text { dos }}$ for electron beams by means of an example. $R_{50, \text { ion }}$ and the reference depth $z_{\text {ref }}$ are also indicated. Note that $z_{\text {ref }}$ in this case does not coincide with the depth of dose maximum.

Note this means that the geometrical centre of the ionization chamber is shifted away from the beam source over a distance of 0.5 times the inner radius of the chamber's cavity. 


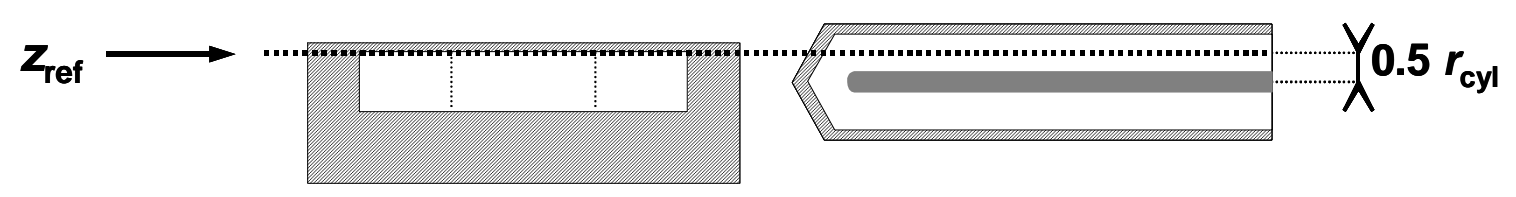

Figure 3: Plane-parallel and cylindrical ionisation chamber each at reference depth $z_{\text {ref }}$.

The parameter $R_{50, \text { dos }}$ is measured under the following reference conditions:

- water phantom,

- constant SSD (source-surface distance) of $100 \mathrm{~cm}$,

- field size at the phantom surface of at least $10 \mathrm{~cm} \times 10 \mathrm{~cm}$ or $20 \mathrm{~cm} \times 20 \mathrm{~cm}$ if $R_{50 \text {,dos }}$ is larger than $7 \mathrm{~cm}$.

The recommendations regarding ionisation chambers, phantoms and sleeves, given in Section 1.5 should be followed. When departing from an ionisation curve, $R_{50, \text { dos }}$ is derived

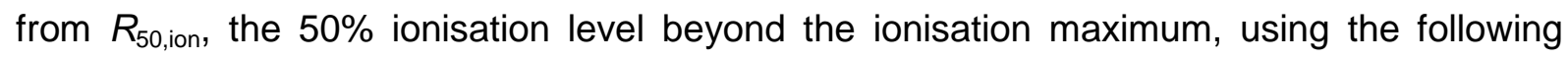
generic expressions [32]:

$$
\begin{array}{ll}
R_{50, \text { dos }}=1.029 \cdot R_{50, \text { ion }}-0.06 \mathrm{~cm} & \left(R_{50, \text { ion }} \leq 10 \mathrm{~cm}\right) \\
R_{50, \text { dos }}=1.059 \cdot R_{50, \text { ion }}-0.37 \mathrm{~cm} & \left(R_{50, \text { ion }}>10 \mathrm{~cm}\right)
\end{array}
$$

For some plane-parallel ionisation chambers it has been reported that recombination and polarity effects vary as a function of depth. It can be concluded that these variations, as defined in Appendix A.5, can appear at different depths and consequently this variation should be investigated and taken into account if there is a variation with depth.

\subsection{Determination of $D_{\mathrm{w}, \mathrm{Q}}$ under reference conditions.}

The formalism to derive the absorbed dose to water for electron beams is described in section 1.3. Detailed information on the uncertainties, calculation of the influence quantities and numerical data needed for $k_{Q}$ or $k_{Q, Q \text { cross }}$ calculation can be found in the Appendices. Moreover, Appendices A.1-A.7 list the quantities, symbols and definitions and expected differences with NCS report 5.

The recommendations are stated below:

1. The local standard shall be either a Farmer type graphite-walled cylindrical ionisation chamber of one of the types listed in Table 1, or a plane-parallel ionisation chamber of type NACP02, PTW 34001 or Wellhöfer PPC-40. Note that plane-parallel chambers must be used for beam qualities $R_{50, \text { dos }}<4 \mathrm{~cm}$. 
2. The Farmer type chamber used as a local standard shall be calibrated in terms of absorbed dose to water, $N_{D, w}$, at a Primary Standard Dosimetry Laboratory (PSDL) ${ }^{*}$ in a ${ }^{60} \mathrm{Co}$ beam under the conditions described in section 1.4. The plane-parallel chamber types used as a local standard shall be preferably cross calibrated against a Farmer type chamber in an electron beam with a beam quality index $R_{50 \text {,dos }}$ greater than $7 \mathrm{~cm}$. Only for the case this option is not available to a particular user, the protocol also provides the data necessary to perform dosimetry using a plane-parallel ionisation chamber based on a calibration in a ${ }^{60} \mathrm{Co}$ reference beam.

3. A water phantom shall be used with a vertical beam; the non-waterproof chamber shall be fitted in water with a thin waterproofing sheath made of PMMA, not thicker than $1 \mathrm{~mm}$. More information on phantoms and sleeves is given in section 1.5.

4. For the determination of the absorbed dose to water in the users beam, the chamber shall be placed on the beam axis with the reference point of the chamber at the reference depth [31]:

$$
z_{\text {ref }}=0.6 R_{50, \text { dos }}-0.1 \mathrm{~cm}
$$

For a cylindrical chamber the reference point has to be taken as 0.5 times the inner radius of the chamber in front of the geometrical centre, whereas for a plane-parallel the reference point of the chamber is taken as the centre of the inner surface of its front window.

5. The water surface shall be positioned at a fixed source-surface distance of SSD = $100 \mathrm{~cm}$. The field size at the phantom surface should be either $10 \mathrm{~cm} \times 10 \mathrm{~cm}$ or the field size used for normalization of output factors, whichever is larger ${ }^{\dagger}$.

6. If the local standard is a Farmer type cylindrical ionisation chamber the absorbed dose to water $D_{w, Q}$ at the reference depth will be given by :

$$
D_{w, Q}=M_{c o r r, Q} N_{D, w} k_{Q}
$$

where:

$$
\begin{aligned}
& D_{w, Q} \quad \text { is the absorbed dose to water in the users beam } Q \text { at the reference } \\
& \text { depth } Z_{\text {ref, }}
\end{aligned}
$$

\footnotetext{
"For the Netherlands the Primary Standard Dosimetry Laboratory (PSDL) is the NMi at Delft whereas for Belgium the Primary Standard Dosimetry Laboratory is the LSDG at Ghent.

${ }^{\dagger}$ Note that this is different than the recommendation for the determination of the beam quality index $R_{50, \text { dos. }}$.
} 
$M_{c o r r, Q}$ is the electrometer reading, corrected for influence quantities as described in Appendix A..2,

$N_{D, w} \quad$ is the absorbed dose to water calibration factor in the ${ }^{60} \mathrm{Co}$ reference beam quality, given by the Standards Laboratory at reference ambient conditions (normally $T_{\text {ref }}=20^{\circ} \mathrm{C}$ and $P_{\text {ref }}=101.325 \mathrm{kPa}$ and $50 \%$ relative humidity),

$k_{Q} \quad$ is the beam quality correction factor that depends on the chamber type and radiation beam quality of the users beam. Recommended $k_{Q}$ values as function of the quality index $R_{50 \text {,dos }}$ and for the three recommended ionisation chamber types are obtained from the following equation:

$$
k_{Q}=A-B \cdot\left[R_{50, \text { dos }}(Q)\right]^{C}
$$

The parameters A, B and C for this equation are given in Table 2. The $k_{Q}$ for the Farmer type chambers are valid in the beam quality range from $4 \mathrm{~cm}$ to $12 \mathrm{~cm}$ The data obtained using equation (11) are plotted in Figure 12 (see Appendix A.5 section A.5.2).

7. If the local standard is a plane-parallel ionisation chamber it is recommended that a calibration coefficient $N_{D, w, Q_{\text {cross }}}^{p p}$ is used, which is obtained in a high energy electron beam $Q_{\text {cross }}$ as explained below. Then the absorbed dose to water $D_{w, Q}$ at the reference depth will be given by:

$$
D_{w, Q}=M_{c o r r, Q}^{p p} \cdot N_{D, w, Q_{\text {cross }}}^{p p} \cdot k_{Q, Q_{\text {cross }}}
$$

where:

$D_{w, Q} \quad$ is the absorbed dose to water in the users beam $Q$ at the reference depth $z_{\text {ref, }}$

$M_{c o r r, Q}^{p p} \quad$ is the electrometer reading, corrected for influence quantities as described in Appendix A.2,

$N_{D, w, Q_{\text {cross }}}^{p p}$ is the absorbed dose to water calibration coefficient in the cross calibration beam quality $Q_{\text {cross }}$ at reference ambient conditions 
(normally $T_{\text {ref }}=20^{\circ} \mathrm{C}$ and $P_{\text {ref }}=101.325 \mathrm{kPa}$ and $50 \%$ relative humidity). $N_{D, w, Q_{c r o s s}}^{p p}$ is obtained from calibrating the plane-parallel chamber (pp) in the beam quality $Q_{\text {cross }}$ against the dose measured by a Farmer type cylindrical ionisation chamber:

$$
N_{D, w, Q_{\text {cross }}}^{p p}=\frac{M_{c o r r, Q_{\text {cross }}}^{c y l}}{M_{c o r r, Q_{\text {cross }}}^{p p}} \cdot N_{D, w}^{c y l} \cdot k_{Q_{c r o s s}^{c y l}}^{c l}
$$

$k_{Q, Q \text { cross }}$ is the beam quality correction factor that depends on the chamber type and radiation beam quality of both the cross calibration beam quality $\mathrm{Q}_{\text {cross }}$ and the users beam quality $\mathrm{Q}$. Recommended $k_{Q, Q \text { cross }}$ values as function of the quality index $R_{50 \text {,dos }}$ and for the recommended ionisation chamber types are obtained from the following equation:

$$
k_{Q, Q_{\text {cross }}}=\frac{A-B \cdot\left[R_{50, \text { dos }}(Q)\right]^{C}}{A-B \cdot\left[R_{50, \text { dos }}\left(Q_{\text {cross }}\right)\right]^{C}}
$$

The parameters for this equation are given in Table 2 . The $k_{Q, Q \text { cross }}$ are valid in the beam quality range from $1 \mathrm{~cm}$ to $12 \mathrm{~cm}$. The data obtained using equation (14) are plotted in figure 13 (see Appendix A.5, section A.5.2).

8. If the local standard is a plane-parallel ionisation chamber, calibrated in terms of absorbed dose to water in a ${ }^{60} \mathrm{Co}$ reference beam: the same procedure as outlined in point 6 should be followed. Note, that in this case the depth has to be corrected for non-water equivalence of the entrance window of the plane-parallel chamber. To this end, the depth at which the outside front face of the plane-parallel ionisation chamber should be positioned equals the reference depth minus the water equivalent thickness of the entrance window as given in Table 3 (see Appendix A.1).

9. The electrometer reading $M_{Q}$ should be corrected for all influence quantities:

$$
M_{\text {corr }, Q}=M_{Q} k_{T P} k_{h} k_{p o l} k_{s}
$$

where: 
$k_{T P}$ is the correction factor for pressure and temperature in the air cavity at the time of measurement,

$k_{h}$ is the correction for humidity in the air cavity at the time of measurement,

$k_{\text {pol }}$ is the correction on the chamber reading due to the applied polarity of the polarizing voltage,

$k_{s} \quad$ is the correction for incomplete charge collection in the ionisation chamber due to recombination.

Determination of these correction factors is explained in Appendix A.2.

10. Under these conditions the relative standard uncertainty of the absorbed dose to water $D_{w, Q}$ at the reference depth $z_{\text {ref }}$ for a high energy electron beam is estimated to be $1.5 \%(1 \mathrm{sd})$ when it concerns a measurement with a Farmer type chamber in an electron beam with $R_{50, \text { dos }}>7 \mathrm{~cm}$ or $1.8 \%$ (1sd) when it concerns a measurement with a cross calibrated plane-parallel chamber in any electron beam. More details on this uncertainty estimate are given in Appendix A.5.

Table 2: Parameters for the model of equations (11) and (14) to obtain $k_{Q}$ and $k_{Q, Q c r o s s}$ values as a function of the beam quality $R_{50 \text {,dos }}$ for the recommended types of ionisation chambers (see Appendix A.5).

\begin{tabular}{|c|c|c|c|}
\hline & A & B & C \\
\hline Farmer types & 0.9345 & 0.0057 & 0.7733 \\
\hline NACP02 & 1.1955 & 0.2274 & 0.1479 \\
\hline Roos types & 1.1376 & 0.1700 & 0.1835 \\
\hline
\end{tabular}

\subsection{Determination of $D_{\mathrm{w}, \mathrm{Q}}$ under non-reference conditions}

Determination of $D_{\mathrm{w}, Q}$ under non-reference conditions is not part of the CoP, however a few points should be considered. Field instruments shall be calibrated against the reference instrument at the radiation qualities at which they are to be used. This calibration shall be in terms of absorbed dose to water.

1. Only ionisation chambers are considered in this protocol.

2. Field ionisation chambers shall be calibrated at the same reference depth $z_{\text {ref }}$, SSD and field size as stated in section 3.3. A water phantom shall be used. This calibration has to be done for each beam quality at which they are to be used. 
The absorbed dose to water calibration coefficient $N_{w, Q}^{\text {field }}$ at the users beam quality $Q$ for the field ionisation chamber is than given by:

$$
N_{w, Q}^{\text {field }}=D_{w, Q}^{s t d} / M_{c o r r, Q}^{\text {field }}
$$

where:

$D_{w, Q}^{s t d}$ is the absorbed dose to water in the users beam at the reference depth $Z_{\text {ref }}$ determined by the local standard,

$M_{c o r r, Q}^{\text {field }}$ is the electrometer reading of the field instrument, corrected for influence quantities as described in Appendix A.2.

Preferably an additional monitor chamber in the radiation field should be used to normalise the reading in order to account for beam fluctuations.

3. If the field instrument is one of the recommended Farmer type ionisation chambers, than this calibration can be considered as a cross calibration and the obtained calibration coefficient $N_{w, Q}^{\text {field }}$ is applicable for other electron beams with $R_{50 \text {,dos }}>4 \mathrm{~cm}$. To this end, a "60${ }^{60}$ calibration coefficient" for the field instrument can be back-calculated from the ratio $N_{w, Q}^{\text {field }}$ and $k_{Q}$. This calibration coefficient can subsequently be used according to the CoP. An additional uncertainty component should be taken into account.

4. Relative measurements (i.e. determination of the absorbed dose at other points in the phantom than $z_{\text {ref }}$ ) can be performed by the same ionisation chambers described above. The effective point of measurement of the ionisation chamber has to be positioned at the depth(s) of interest. Since the water to air mass stopping power ratios vary considerably as a function of electron energy the measured depth ionisation curve has to be converted into a depth dose curve by applying the water-to-air mass stopping power ratios given by the formula in section A 5.5. It has also been reported that for some ionisation chambers the recombination and polarity corrections may vary considerably as a function of depth. Consequently, these influence quantities (see Appendix A.2) should be measured as a function of depth and be corrected for if necessary.

5. The use of other dosimetry detectors (i.e. diodes, diamond detectors, TLD, Alanine, scintillator detectors, etc.) is not within the scope of this report. 


\section{Appendices}

A.1 Instrument data for recommended ionisation chambers

In Table 3 the characteristics are given for the ionisation chambers recommended for reference dosimetry in this code of practice. 
Table 3: Characteristics of ionisation chamber types recommended for reference dosimetry ${ }^{1}$

data taken from manufacturers

${ }^{2}$ Polymethyl Methacrylate $\left(\mathrm{C}_{5} \mathrm{H}_{8} \mathrm{O}_{2}\right)$, also known as acrylic. Trade names are Lucite, Perspex or Plexiglass.

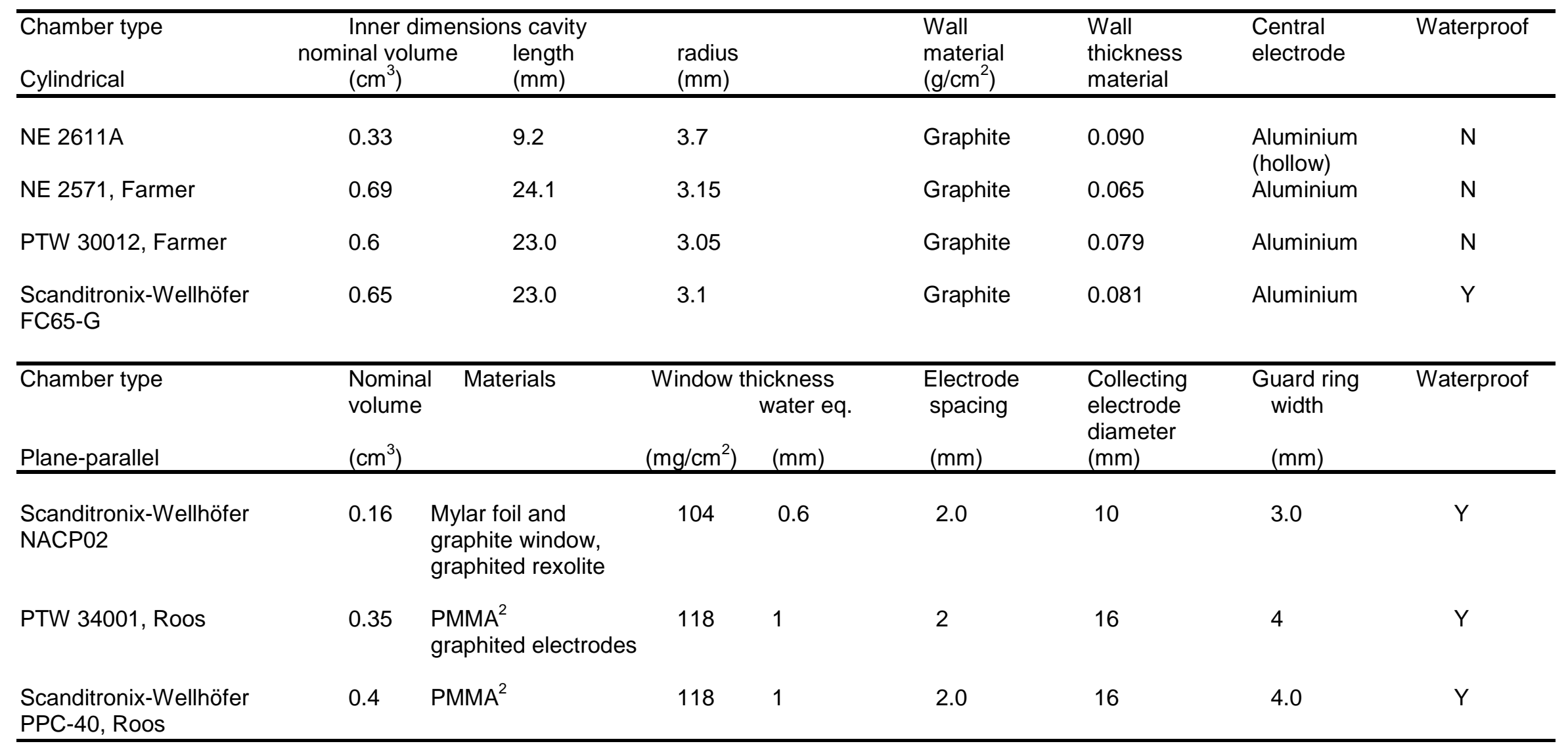




\section{A.2 Influence parameters}

\section{Air density correction}

The ionisation chambers recommended for reference dosimetry in this Code of Practice are open to ambient air. Consequently the mass of air in the cavity will vary with atmospheric conditions for temperature and pressure. The correction factor $k_{T P}$ given by:

$$
k_{T P}=\frac{(T+273.15)}{\left(T_{0}+273.15\right)} \frac{P_{0}}{P}
$$

accounts for this effect by converting the mass of air in the cavity to the reference conditions for temperature $T_{0}=20^{\circ} \mathrm{C}$ and pressure $P_{0}=101.325 \mathrm{kPa}$.

\section{Humidity}

No correction is made for the relative humidity, if the ionisation chamber is used in a range of $20 \%$ to $80 \%$ relative humidity and has been calibrated at a reference condition for relative humidity of $50 \%$ [34]. It is recommended to use ionisation chambers in relative humidity conditions between 20 and $80 \%$. In the unlikely case that the relative humidity is outside this range, a correction factor has to be applied.

\section{Electrometer calibration coefficient $k_{\text {elec }}$}

It is common practice in Belgium and the Netherlands to calibrate the ionisation chamber and the electrometer together, as one set. In this case the calibration coefficient $N_{D, w}$ is expressed in the unit Gy/rdg or Gy/C depending on the readout of the electrometer and no separate electrometer calibration coefficient has to be applied. If the ionisation chamber and electrometer are calibrated separately, the calibration coefficient $N_{D, w}$ for the ionisation chamber is given in units $\mathrm{Gy} / \mathrm{C}$. The calibration coefficient obtained for the electrometer converts the electrometer reading to charge and is expressed in unit $\mathrm{rdg} / \mathrm{C}$. If the reading of the electrometer is in terms of charge the electrometer calibration coefficient is dimensionless.

\section{Polarity correction}

The reading of an ionisation chamber is affected by the polarity of the chamber voltage. The polarity effect depends on the radiation quality, the (incident) beam energy, the magnitude of the polarizing voltage on the chamber, the field size and depth in the phantom, and the 
design of the ionisation chamber. The effect may be considerable for plane-parallel ionisation chambers in electron beams, more in particular in low energy electron beams. The polarity effect for every reference chamber should always be measured at the reference point in the energy range where the chamber is to be used. If the polarity correction is more than a few percent the chamber is not recommended for reference dosimetry. The correction factor for polarity in a given radiation beam is defined by:

$$
k_{\text {pol }}=\frac{\left|M^{+}\right|+\left|M^{-}\right|}{2 M}
$$

where the superscripts + and - in the nominator indicate the electrometer reading with a positive and negative polarizing voltage respectively. $M$ is the electrometer reading taken at polarity routinely used during measurements. When the chamber is sent for calibration, the calibration certificate should clearly state the polarizing voltage and the polarity adopted during calibration and if or not a polarity correction has been applied by the calibration laboratory. If a chamber is used in a radiation beam with nearly the same quality as the reference beam in the calibration laboratory, and with the same magnitude and sign of the polarizing voltage, the polarity is the same and no correction is necessary. For most chamber types the correction is small in photon beams and is often neglected (The primary standards laboratories in Belgium and the Netherlands, LSDG and NMi, don't apply a polarity correction during calibration in their reference ${ }^{60} \mathrm{Co}$ beams).

When the calibration laboratory has applied a polarity correction during calibration at the reference beam quality (usually ${ }^{60} \mathrm{Co}$ ) the user has to determine $k_{\text {pol }}$ according to equation (18) for all measurements made using the routine polarity. When the calibration laboratory has not applied a polarity correction then equation (18) has to be modified to evaluate the polarity effect in a user beam with quality $Q$. The polarity correction for beam quality $Q$ has to be determined relative to the polarity correction of the reference beam quality ${ }^{60} \mathrm{Co}$ using the expression:

$$
k_{\text {pol }}=k_{\text {pol,Q }} / k_{\text {pol,Co }}
$$

The correction $k_{\text {pol,Co }}$ has to be determined either by the user from known data of the chamber response to different beam qualities and polarities with a relative standard 
uncertainty of less than $0.5 \%$ or by sending the chamber to a calibration laboratory in order to measure the polarity correction in $\mathrm{a}^{60} \mathrm{Co}$ beam.

\section{Recombination correction}

A correction is required to account for the lack of complete charge collection in the chamber volume, due to the recombination of ions. Several physical phenomena may contribute to recombination, but initial and volume or general recombination are commonly considered the most important effects [35]. Initial recombination refers to the forming of ion pairs along the track of a single ionising particle and therefore independent of the dose rate. Volume recombination is due to recombination of ions from different tracks and depends on the ion density and thus on the dose rate (continuous beams) or dose per accelerator pulse (pulsed beams). Both effects depend on the chamber geometry and on the polarizing voltage applied to the chamber.

For continuous radiation beams, i.e. ${ }^{60} \mathrm{Co}$, the recombination effect is small $(<0.2 \%)$ for the output rates encountered at irradiation facilities in calibration laboratories. Often initial recombination is neglected and only general recombination is considered assuming a linear relation between the inverse of the charge $(1 / M)$ and the inverse square of the polarizing voltage $\left(1 / U^{2}\right)$. However, when a proper correction is required initial recombination in continuous beams should not be neglected. In this case $k_{s}$ can be approximated by using a method described by Boutillon [37]. This method evaluates the recombination correction including both the initial and general recombination component.

In pulsed radiation beams general recombination is the dominant effect. The recombination correction may amount to $1 \%-3 \%$ for a high dose rate beam produced by a medical linear accelerator and even higher for pulsed-scanned beams. An expression for the recombination factor can be derived using a theoretical approach developed by Boag [38, 39]. For pulsed beams the correction for volume recombination can be written as:

$$
k_{s}=1 / f=u / \ln (1+u)
$$

The dimensionless variable $u$ is given as $u=\mu m d^{2} / U$, where $m$ is the dose (charge density) per pulse, $d$ is the electrode separation in the ionisation chamber, $U$ the applied polarizing voltage and $\mu$ is a constant depending on the ion mobilities and recombination rate of the gas in the chamber volume. For cylindrical ionisation chambers the effective electrode separation can be calculated from expressions given by Boag [35]. However, the method does not account for chamber-to-chamber variations within a given chamber type. Under near 
saturation conditions $\left(k_{s}<1.05\right)$ the recombination correction for pulsed and pulsed-scanned beams can be accurately determined using the two-voltage method as described by Boag and Currant [36]. The two-voltage technique involves the measurement of charge produced by a radiation beam in the cavity of an ionisation chamber when two different polarizing voltages are applied to the ionisation chamber. The method assumes a linear relation between $1 / M$ and $1 / U$. The recombination correction is determined from the values of charges or readings $M_{1}$ and $M_{2}$, which are collected in the same irradiation conditions at the normal polarizing voltage $U_{1}$ and at a lower voltage $U_{2}$ respectively. The ratio $\left(U_{1} / U_{2}\right)$ should have a value of at least two or three.

Weinhous and Meli [40] have derived numerical solutions for the evaluation of the recombination correction according to approach of Boag and Currant and have computed quadratic fits to these solutions as a function of the polarizing voltage ratio. The recombination correction factor $k_{s}$ at the normal polarizing voltage $U_{1}$ is obtained from:

$$
k_{s}=a_{0}+a_{1}\left(M_{1} / M_{2}\right)+a_{2}\left(M_{1} / M_{2}\right)^{2}
$$

where $M_{1}$ and $M_{2}$ are the measured charge readings at polarizing voltages $U_{1}$ and $U_{2}$, respectively. In Table 4 the coefficients $a_{i}$ are given for pulsed beams as a function of voltage ratio $\left(U_{1} / U_{2}\right)$. A voltage ratio equal to or larger than 3 is recommended. Note, that the polarity effect will change with the applied polarizing voltage and that $M_{1}$ and $M_{2}$ should be corrected for the change in this effect.

Table 4: Quadratic fit coefficients for pulsed radiation as a function of the voltage ratio $U_{1} / U_{2}$ ). Data are taken from Weinhous and Meli [40].
$\left(U_{1} / U_{2}\right)$
$a_{\circ}$
$a_{1}$
$\mathrm{a}_{2}$

\begin{tabular}{llll}
\hline 2.0 & 2.337 & -3.636 & 2.299 \\
2.5 & 1.474 & -1.587 & 1.114 \\
3.0 & 1.198 & -0.875 & 0.677 \\
3.5 & 1.080 & -0.542 & 0.463 \\
4.0 & 1.022 & -0.363 & 0.341 \\
5.0 & 0.9745 & -0.1875 & 0.2135 \\
6.0 & 0.9584 & -0.1075 & 0.1495 \\
8.0 & 0.9502 & -0.03732 & 0.08750 \\
10.0 & 0.9516 & -0.01041 & 0.05909
\end{tabular}


For values of $k_{s}<1.03$ the recombination correction can be approximated to within $0.1 \%$ by the expression [41]:

$$
k_{s}-1=\frac{M_{1} / M_{2}-1}{U_{1} / U_{2}-1}
$$

Note that for a recombination correction $<1.03$ equations (21) and (22) will also include the component for initial recombination, which is also proportional to $1 / U$.

The calibration certificate issued by the standards laboratory should state whether or not a recombination correction has been applied. If a calibration laboratory has not applied a recombination correction, the correction factor for the user beam quality $Q$ has to be derived relative to the appropriate correction factor for the reference beam quality ${ }^{60} \mathrm{Co}$ used in the calibration laboratory. The recombination correction is then obtained by:

$$
k_{s}=k_{s, Q} / k_{s, C o}
$$

For ${ }^{60} \mathrm{Co}$ the recombination correction will be relatively small compared to the correction for the user beam quality $Q$ and is therefore neglected in most cases. Parallel-plate chambers used for electron beams having typically plate separations of $1-2 \mathrm{~mm}$ would be expected to have a low recombination effect, when employed in clinical beams. However, some recombination corrections measured using the two-voltage method are larger than expected. It has been demonstrated that for some plane-parallel chambers the linear relationship between $1 / \mathrm{M}$ and $1 / \mathrm{U}$ is not satisfied in the voltage region used for the two-voltage method [36, 40, 42-44]. It is recommended only to apply the two-voltage method to plane parallel chambers, if the response of the chamber as function of the polarizing voltage has been measured up to its maximum value specified by the manufacturer in order to establish a range of linearity. The chamber should be operated at voltages to remain within the linear range, ensuring that the use of the two-voltage method is valid. 


\section{A.3 Absorbed dose to water standards at NMi and LSDG}

The Belgian and Dutch standard dosimetry laboratories provide absorbed dose to water calibration coefficients in ${ }^{60} \mathrm{Co}$ relying on water calorimeters. For high energy photon beams $k_{Q}$ values, NCS decided to base these on experimental data. To this end experimental $k_{Q}$ 's were measured in nine clinical photon beams using the NMi portable water calorimeter.

This section describes the water calorimeters of both laboratories. The experimental determination of $k_{Q}$ values with the $\mathrm{NMi}$ water calorimeter and the analysis of the experimental $k_{Q}$ values in the context of this NCS protocol are described in Appendix A.4.

\section{A.3.1 The NMi water calorimeter}

The design of the water calorimeter is based on the sealed-water calorimeter of Domen [21]. The water calorimeter consists of a water tank surrounding a high-purity water cell, which contains two thermistor probes. The high-purity water cell is a sealed, thin-walled glass vessel enclosing a volume of water. The temperature rise due to irradiation by high energy photons is measured inside this volume of water. The quality of the water is carefully controlled by purifying and by saturating the water with selected gasses, before filling the glass vessel. The water calorimeter is compact and transportable, weighing $60 \mathrm{~kg}$ when empty, and having outer dimensions of only $60 \mathrm{~cm} \times 60 \mathrm{~cm} \times 70 \mathrm{~cm}$. The dimensions of the water phantom are standard $30 \mathrm{~cm} \times 30 \mathrm{~cm} \times 30 \mathrm{~cm}$. A small spacing between the inner and outer polystyrene foam insulation boxes encloses a copper heat exchange system. The six copper walls are connected in a parallel manner, which helps to reduce temperature gradients inside the water phantom. Cooling is performed by a computer-controlled watercooling thermostat, which uses two PT100 thermometers (one mounted on one of the copper walls and one mounted inside the water phantom) to control and monitor the calorimeter temperature. A built-in magnetic stirrer enables a reduction of temperature drifts due to conduction in between irradiation runs. A cold finger placed inside the water phantom can be switched into the cooling circuit to reduce the time needed to cool down from room temperature to $4^{\circ} \mathrm{C}$.

The water calorimeter can be used in both horizontal and vertical beams. A schematic drawing of the NMi water calorimeter is given in Figure 4 below. 


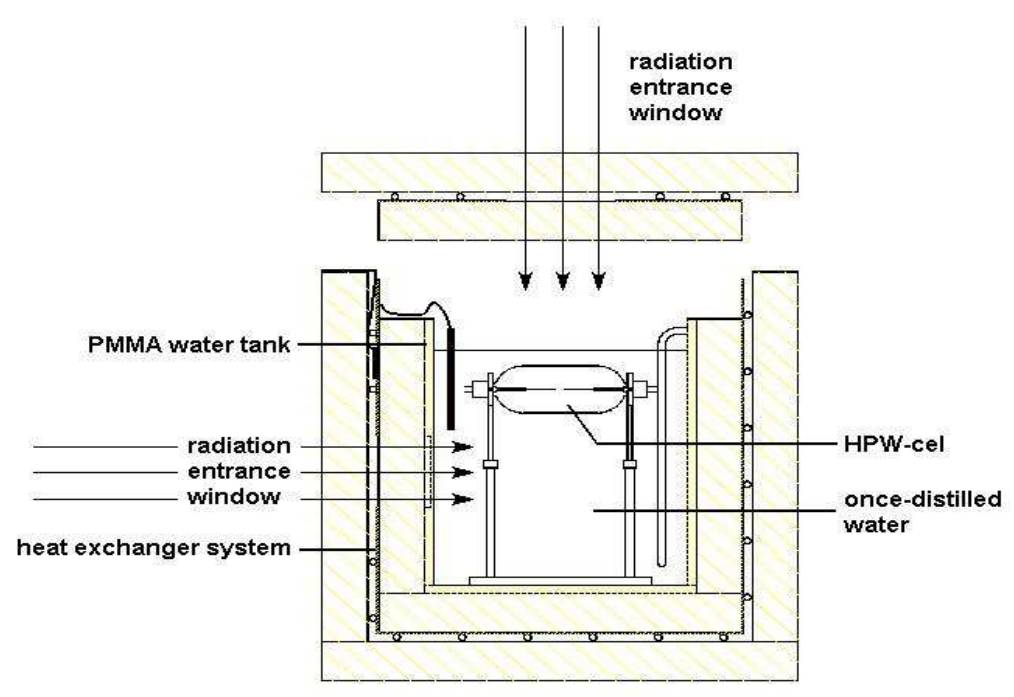

Figure 4: Schematic diagram of the NMi portable water calorimeter which was used for the measurement of $k_{Q}$ values described in appendix A.4.

The calorimeter is equipped with an electrical measurement assembly comprising high precision digital multimeters (DMM) as read-out devices for the determination of the resistance changes of the thermistors during irradiation. Further details are given in [45, 46]. The water calorimeter has been compared with the graphite calorimeter of NMi for ${ }^{60} \mathrm{Co}$ gamma radiation. To convert the dose to graphite measured with the graphite calorimeter to dose to water the fluence scaling theorem is used [45]. Various correction factors of the water calorimeter as a function of photon energy have been determined experimentally and with the use of a computer program to simulate heat transport. Further test measurements were carried out in clinical photon beams in several Belgian hospitals and at NKI with the water calorimeter.

\section{A.3.2 The LSDG water calorimeter}

The construction of the sealed water calorimeter at the university of Ghent is based on the design of Domen [21] and has been described in detail in [47-50]. A short description is given here. The calorimeter tank consists of a $30 \mathrm{~cm} \times 30 \mathrm{~cm} \times 30 \mathrm{~cm}$ water phantom thermally isolated by polystyrene foam as shown in Figure 5 a. 
(a)

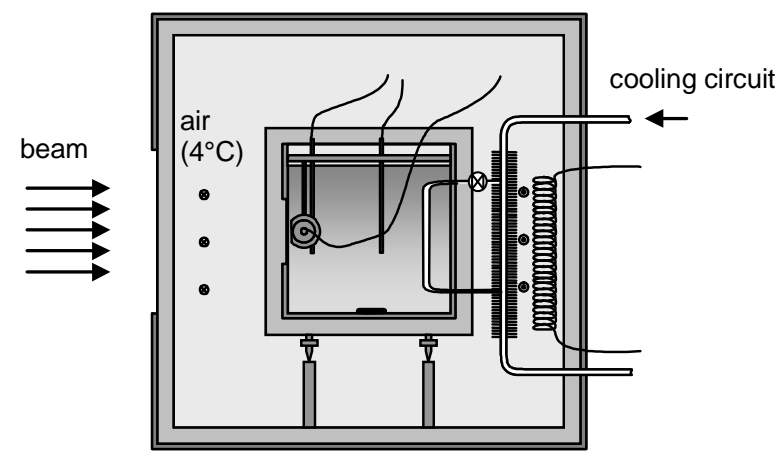

(b)

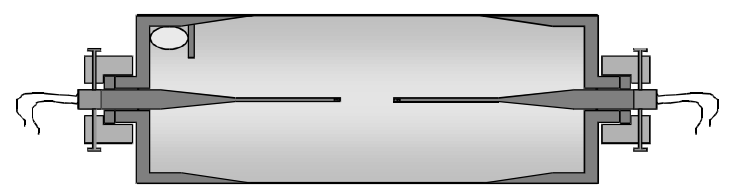

Figure 5: (a) SWC phantom and wooden enclosure of the Ghent water calorimeter and (b) cylindrical glass vessel with thermistor probes positioned (adapted from ref [50].

Isolation of the enclosure allows temperature stabilization of the air surrounding the calorimeter phantom. The operating temperature is $4{ }^{\circ} \mathrm{C}$ in order to remove any concern related to convection. The water temperature is continuously measured with calibrated Ptresistor probes inserted at different positions in the water phantom. The temperature increase due to irradiation is measured at the centre of a cylindrical vessel using small thermostats that are embedded in the tip of small glass probes as shown in Figure $5 \mathrm{~b}$. The probe consists of thermostats embedded in glass rods with a diameter of $0.5 \mathrm{~mm}$. The vessel contains de-ionised and 3 times distilled water and has a diameter of $4 \mathrm{~cm}$, a length of $14 \mathrm{~cm}$ and a wall thickness varying from $0.3 \mathrm{~mm}$ in the central part corresponding with the central axis of the beam to $1.6 \mathrm{~mm}$ at the lateral edges. The water inserted in the vessel is saturated with argon, nitrogen or hydrogen, three systems that do not result in a chemical heat defect. The electronics and the methods to determine the measured temperature increase are described in $[47,48]$.

\section{A.3.3 Experimental Calorimetric determination of absorbed dose to water}

Absorbed dose to water $D_{\mathrm{w}}$ is derived from the temperature increase $\Delta T$ due to irradiation at the location of the thermistors [50] as:

$$
D_{\mathrm{w}}=c_{\mathrm{w}} \cdot \Delta T \cdot k_{\mathrm{c}} \cdot k_{\mathrm{sc}} \cdot k_{\mathrm{dd}} \cdot \frac{1}{1-h}
$$


where $c_{w}$ is the specific heat capacity of water at the measuring temperature, $k_{\mathrm{c}}$ a correction factor for conductive heat effects (which is calculated by numerically solving the heat transport equation), $k_{s c}$ a correction factor for scattering and attenuation induced by probes and vessel (which for the thermistors is negligible and for the vessel is determined by a perturbation measurement), $k_{d d}$ a correction factor for the non-uniformity of the lateral dose distributions and $h$ the chemical heat defect which corrects for the heat that is released or absorbed in chemical reactions by radiation induced species with each other or with impurities present in the water. The latter is calculated by numerically solving the set of coupled differential equations that describe all chemical reactions taking place and requires knowledge of the enthalpy of formation of each species involved in the reactions and is studied in detail in $[47,48]$. In Tables $5 \mathrm{a}$ and $5 \mathrm{~b}$ the uncertainty budgets for the determination of absorbed dose to water in $\mathrm{a}^{60} \mathrm{Co}$ photon beam for respective the LSDG and the NMi water calorimeter are shown.

Table 5a: Uncertainty budget for the determination of absorbed dose to water $D_{w, Q}$ in $a^{60}$ Co photon beam with the LSDG water calorimeter

\begin{tabular}{|c|c|c|}
\hline $\begin{array}{c}\text { Input quantity } x_{i} \\
\text { Source of unceratinty }\end{array}$ & $\begin{array}{l}\text { Uncertainty } \\
\text { type }\end{array}$ & $\begin{array}{c}\text { Standard } \\
\text { uncertainty }\end{array}$ \\
\hline & & $u_{i}$ \\
\hline \multicolumn{3}{|c|}{ Dose determination water calorimeter $D_{w}$ in ${ }^{60} \mathrm{Co}$} \\
\hline Reproducibility calorimeter response & A & $0.20 \%$ \\
\hline Bridge calibration & A & $0.20 \%$ \\
\hline Thermistor calibration & B & $0.15 \%$ \\
\hline Thermistor positioning & B & $0.13 \%$ \\
\hline Excess heat correction factor $k_{c}$ & B & $0.25 \%$ \\
\hline Scatter correction factor $\mathrm{k}_{\mathrm{sc}}$ & $\mathrm{B}$ & $0.04 \%$ \\
\hline Chemical heat defect $\mathrm{h}$ & $\mathrm{B}$ & $0.50 \%$ \\
\hline Combined Standard Uncertainty in $D_{w} 0$ & & $0.66 \%$ \\
\hline
\end{tabular}

Note that the uncertainty analysis for the LSDG water calorimeter is still based on the comparison held at the BIPM in September 1999 [66]. Further work is foreseen to reevaluate the uncertainty budget in the future. 
Table 5b: Uncertainty budget for the determination of absorbed dose to water $D_{w, Q}$ in ${ }^{60} \mathrm{Co}$ photon beam with the NMi portable water calorimeter

\begin{tabular}{|l|l|c|c|}
\hline \multicolumn{1}{|c|}{$\begin{array}{c}\text { Input quantity } X_{i} \\
\text { Source of uncertainty }\end{array}$} & $\begin{array}{c}\text { Uncertainty } \\
\text { type }\end{array}$ & $\begin{array}{c}\text { Standard } \\
\text { uncertainty }\end{array}$ \\
\hline & \multicolumn{1}{|c|}{$u_{i}$} \\
\hline Dose determination water calorimeter $\boldsymbol{D}_{\boldsymbol{w}}$ in ${ }^{60} \mathbf{C o}$ & & \\
\hline & Reproducibility calorimeter response & $\mathrm{A}$ & $0.26 \%$ \\
\hline & Long-term stability in ${ }^{60} \mathrm{Co}$ & $\mathrm{A}$ & $0.18 \%$ \\
\hline & Thermistor calibration & $\mathrm{B}$ & $0.14 \%$ \\
\hline & Thermistor positioning & $\mathrm{B}$ & $0.10 \%$ \\
\hline & Excess heat correction factor $\mathrm{k}_{\mathrm{c}}$ & $\mathrm{B}$ & $0.10 \%$ \\
\hline & Scatter correction factor $\mathrm{k}_{\mathrm{sc}}$ & $\mathrm{B}$ & $0.15 \%$ \\
\hline & Chemical heat defect h & $\mathrm{B}$ & $0.20 \%$ \\
\hline & & & \\
\hline & Combined Standard Uncertainty in $\boldsymbol{D}_{w, Q}$ & & $0.45 \%$ \\
\hline
\end{tabular}

\section{A.4 Experimental $k_{\mathrm{Q}}$ values and data for clinical high energy photon beams}

\section{A.4.1 Description of beams and ionisation chambers}

$k_{Q}$ values were measured for four cylindrical ionisation chamber types in 9 clinical high energy photon beams using the NMi portable water calorimeter. Details of these beams are shown in Table 6 . The beam qualities were measured using a uniform protocol that is consistent with the recommendations of this CoP and TRS-398 for TPR 20,10 and TG-51 for $\% d d(10)_{x}$.

For each chamber type, 6 individual chambers were used. Details of the chamber types can be found in Table 3 (appendix A.1). They are all graphite-walled ionisation chambers with an aluminum central electrode. These characteristics fulfil the criteria for robustness and stability for reference ionisation chambers as described in TRS-398. 
Table 6: The clinical beams in which $k_{Q}$ were measured with their photon beam quality specifiers $\mathrm{TPR}_{20,10}$ and $\% d d(10)_{\mathrm{x}}$.

\begin{tabular}{|c|l|c|l|c|c|}
\hline Date & Accelerator & $\begin{array}{c}\text { Photon } \\
\text { Energy } \\
(\mathrm{MV})\end{array}$ & Institute & TPR 20,10 & $\% d d(10)_{\times}$ \\
\hline \hline $03 / 03$ & Varian 2300 C/D & 15 & Jolimont, Haine Saint-Paul, BE & 0.7576 & 77.97 \\
\hline $06 / 03$ & Elekta SI15 & 15 & AMC, Amsterdam, NL & 0.7287 & 73.06 \\
\hline $06 / 03$ & Elekta SI25 & 25 & UCL, Brussels, BE & 0.7993 & 84.38 \\
\hline $06 / 03$ & Elekta SI18 & 15 & AZ St Luc, Gent, BE & 0.7583 & 76.84 \\
\hline $09 / 03$ & Siemens Primus & 18 & Virga Jesse Ziekenhuis, Hasselt, BE & 0.7674 & 79.52 \\
\hline $09 / 03$ & Elekta SI15 & 6 & NKI/AvL, Amsterdam, NL & 0.6732 & 66.24 \\
\hline $12 / 03$ & Siemens Primus & 23 & Service de Radiotherapie, CHU de Liège, BE & 0.7829 & 81.39 \\
\hline $01 / 04$ & Varian 2300 C/D & 6 & VU, Amsterdam, NL & 0.6594 & 65.82 \\
\hline \hline $11 / 04$ & Varian 2300 C/D & 15 & $\begin{array}{l}\text { Jolimont, Haine Saint-Paul, BE (repeated } \\
\text { measurement) }\end{array}$ & 0.7563 & 78.05 \\
\hline
\end{tabular}

\section{A 4.2 Experimental method}

The beam quality correction factor $k_{Q}$ is defined as the ratio of absorbed dose to water calibration coefficients in the clinical beam quality $Q, N_{D, w, Q}$ and the reference (calibration) beam quality $Q_{0}, N_{D, w}\left({ }^{60} \mathrm{Co}\right.$ in this case and therefore $Q_{0}$ is omitted):

$$
k_{Q}=\frac{N_{D, w, Q}}{N_{D, w}}
$$

The absorbed dose to water calibration coefficients are determined with the portable water calorimeter. For the beam quality $Q$ :

$$
N_{D, w, Q}=\frac{D_{w, Q}}{M_{c o r r, Q}}
$$

where:

$N_{D, w, Q}$ calibration coefficient for reference chamber in beam quality $Q$,

$D_{w, Q} \quad$ absorbed dose to water per monitor unit for beam quality $Q$,

$M_{\text {corr, } Q}$ reference chamber signal per monitor unit.

Absorbed dose to water per monitor unit in the accelerator beam is derived as: 
$D_{w, Q}=\frac{1}{M_{m o n, Q} \cdot k_{T P, m o n, Q}} \cdot \Delta R_{\text {midrun }, Q} \cdot \frac{d T}{d R}\left(T_{Q}\right) \cdot c_{w}\left(T_{Q}\right) \cdot\left(\Pi k_{i}\right)_{Q}$

where (for the beam quality $Q$ ):

$M_{m o n, Q} \cdot k_{T P, m o n, Q}$ monitor signal corrected for $\mathrm{T}$ and $\mathrm{P}$,

$\Delta R_{\text {midrun, } Q} \quad$ thermistor resistance change as a result of irradiation,

$T_{Q} \quad$ calorimeter operating temperature at the point of measurement,

$d T / d R \quad$ thermistor response as function of thermistor resistance,

$c_{w}\left(T_{Q}\right) \quad$ specific heat capacity of water at the working temperature,

$\left(\Pi k_{i}\right)_{Q} \quad$ product of correction factors for the chemical heat defect, for excess heat due to the presence of non-water materials, for the perturbation due to presence of the glass cell and for deviations from the reference SDD and measurement depth.

The overall measurement equation to derive $k_{\mathrm{Q}}$ thus becomes:

$$
k_{Q}=\frac{M_{\text {corr }, Q}}{M_{\text {corr },{ }^{60} C_{C o}}} \cdot \frac{M_{\text {mon }^{60}{ }_{C o}} \cdot k_{P T, \text { mon },}^{60}{ }_{C o}}{M_{\text {mon, }, Q} \cdot k_{P T, m o n, Q}} \cdot \frac{\Delta R_{\text {midrun }, Q} \cdot \frac{d T}{d R}\left(T_{Q}\right) \cdot c_{w}\left(T_{Q}\right)}{\Delta R_{\text {midrun },}^{60}{ }_{C o} \cdot \frac{d T}{d R}\left(T_{60}\right) \cdot c_{w o}\left(T_{60}\right)} \cdot \frac{\left(\Pi k_{i}\right)_{Q}}{\left(\Pi k_{i}\right)_{60}{ }_{C o}}
$$

Note that a number of the factors occur both in numerator and denominator and these pairs are strongly correlated.

\section{A.4.3 Uncertainties}

Table 7 summarizes the uncertainty contributions in the experimental determined $k_{Q} . A$ expanded standard uncertainty (coverage factor $\mathrm{k}=2$ ) in $k_{Q}$ of $1.1 \%$ is estimated, These uncertainty estimates apply to the portable water calorimeter, which was used during the measurement campaign in clinical photon beams in Belgium and The Netherlands. Correlations due to common uncertainty contributions are removed from the experimental data presented in Table 7. 
Table 7: Combined standard uncertainty for experimental determined $k_{Q}$ values.

\section{Step 1: Absorbed dose determination in ${ }^{60} \mathrm{Co}$}

Type of physical quantity or procedure

$D_{w}$ determination with water calorimeter

Charge measurement and experimental procedure

Combined uncertainty in $D_{w, C o-60}$

\section{Relative Standard}

Uncertainty (\%)

0.40

0.16

0.43

\section{Step 2: Absorbed dose determination in high energy photon} beam

Type of physical quantity or procedure

$D_{w}$ determination with water calorimeter

Charge measurement and experimental procedure

Combined uncertainty in Step 2

0.33

Combined standard uncertainty in $k_{Q}$ (Steps $\left.1+2\right)$

\section{A.4.4 Results of NCS measurement data}

Table 8 lists the final data of the measurements. Each data point is the average value for the six ionisation chambers of the same type. The standard deviations on these six values was lower than $0.1 \%$ for all data points with the exception of two for which it was $0.2 \%$. The measurement in Jolimont was performed twice and the agreement was good as can be seen in Table 8. In the further analysis the average result was used.

Table 8: Average $k_{Q}$ per chamber type measured with the NMi portable water calorimeter in each of the high energy clinical photon beams.

\begin{tabular}{|l|c|c|c|c|c|c|}
\hline Institute & TPR $_{20,10}$ & $\% d d(10)_{x}$ & NE & NE & PTW & Welhöfer \\
& & & 2571 & 2611 & 30012 & FC65G \\
\hline \hline VU, Amsterdam, NL & 0.6594 & 65.82 & 0.9940 & 1.0013 & 0.9932 & 0.9943 \\
\hline AvL/NKI, Amsterdam, NL & 0.6732 & 66.24 & 0.9966 & 1.0006 & 0.9963 & 0.9996 \\
\hline AMC. Amsterdam, NL & 0.7287 & 73.06 & 0.9840 & 0.9856 & 0.9778 & 0.9837 \\
\hline Jolimont, Haine Saint-Paul, BE & 0.7563 & 78.05 & 0.9667 & 0.9703 & 0.9643 & 0.9679 \\
\hline Jolimont, Haine Saint-Paul, BE & 0.7576 & 77.97 & 0.9681 & 0.9681 & 0.9655 & 0.9690 \\
\hline AZ St Luc, Gent, BE & 0.7583 & 76.84 & 0.9789 & 0.9786 & 0.9771 & 0.9803 \\
\hline Virga Jesse Ziekenhuis, Hasselt, BE & 0.7674 & 79.52 & 0.9769 & 0.9752 & 0.9738 & 0.9752 \\
\hline Service de Radiotherapie, CHU de Liège, BE & 0.7829 & 81.39 & 0.9636 & 0.9690 & 0.9617 & 0.9632 \\
\hline UCL, Brussels, BE & 0.7993 & 84.38 & 0.9654 & 0.9676 & 0.9634 & 0.9661 \\
\hline \hline
\end{tabular}


The data in Table 8 show no significant systematic trends in the differences between the Farmer types: NE 2571, PTW 30012 and Wellhöfer FC65G, which is not unexpected given the similarity in geometry and design of those three chamber types. In the subsequent analysis which will be discussed below, it was also found that the fits to the data for these three chamber types did not show significant differences. It was decided to pool the data for these three chamber types and to recommend a single data-set. An additional reason for that was that for the PTW 30012 and Wellhöfer FC65G chamber types no other data are available from the literature whereas for the NE 2571 there is a substantial amount of data available. The experimental $k_{Q}$ data obtained in that way are presented in Figure 6.
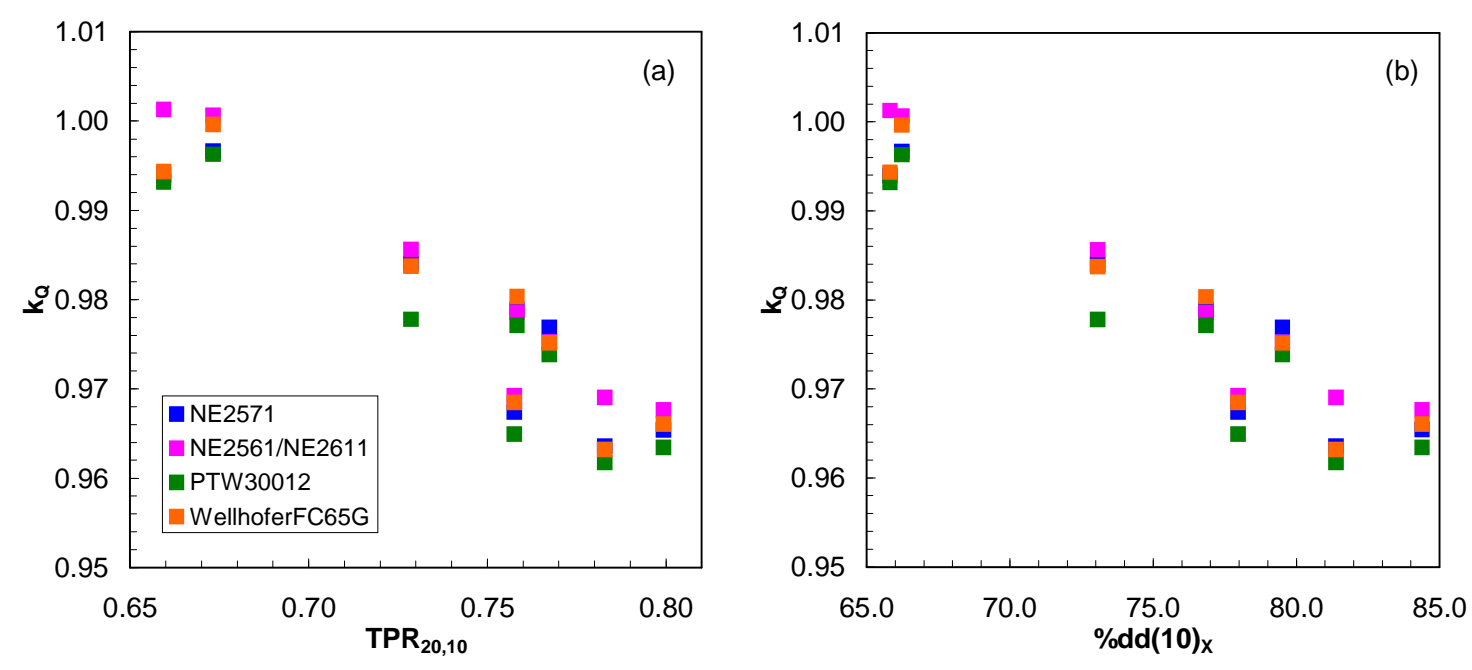

Figure 6: Results of the $k_{Q}$ measurements for the Farmer types and the NE 2611A / NE 2561 types plotted (a) as a function of $\mathrm{TPR}_{20,10}$ and (b) as a function of $\% d d(10)_{x}$.

\section{A.4.5 Analysis of the experimental $k_{Q}$ values}

The data in Figure 6 are found to be insufficient and show too much scatter to be proposed as the single database for this protocol. Therefore, they are analysed in conjunction with other experimental data from the literature.

\section{A.4.5.1 Compilation of experimental $k_{Q}$ values from the literature}

Only for the NE 2571 and NE 2561 / NE 2611A type chambers there is information available from the literature. All the $k_{Q}$ values that were used in this analysis are listed in Table 9. It was decided that only data with an agreed quality standard and for heavily filtered ("cliniclike") beams should be used for the determination of generic experimental $k_{Q}$ data. The first criterion has been interpreted in TRS-398 as data measured by a primary standard 
laboratory for a substantial number of chambers. This requirement is relaxed here such as to include the data measured by Palm et al. [51] and Medin et al. [52] since we did not find an argument that they would not have been measured up to the metrological quality achieved in a standards laboratory. On the contrary, given that they are measured in clinical beams they are more relevant to be included than some data measured in standard laboratories and their inclusion makes the generic set of $k_{Q}$ values more robust. There has been some argument that data measured for a large number of chambers should be assigned a higher weight than other data for smaller sets of chambers. However, the NPL data as well as the NCS data show that chamber to chamber variations are small and that the spread of data in the entire set is more likely due to the differences in measurement method (within similar uncertainty limits) and the variety of beams. Hence, all the data points are given an equal weight in the further analysis. The data for the NE 2561 chambers from Palmans (1999) [49] were excluded since they were not obtained by direct measurement against a water calorimeter. The second criterion (to accept only heavily filtered beam data) excludes three data points from Ross et al. (1994) [54] and the data points by Seuntjens et al. (2000) [55]. Some of the data were measured using the Fricke ferrous sulphate chemical dosimeter of which Seuntjens et al. [55] have demonstrated that its response is not energy independent in the high energy x-ray range. Those results (Shortt et al. (1992) [56], Boutillon et al. (1994) [57], Ross et al. (1994) [54] and Guerra et al. (1995) [58]) have been corrected for this dependence with the equation presented by Seuntjens et al. [55]. For the LNHB data taken from Andreo (2000) [60] the values were back-corrected with the expression given by Andreo (2000) [60] and corrected with the expression given by Seuntjens et al.[55]. The Palm et al. (2002) [51] data have also been measured with Fricke but the results were already corrected in the same way by the authors.

For most of the data in this compilation the sleeve material was PMMA and the sleeve thickness was $1 \mathrm{~mm}$ but not for all data this information is given in the cited publications. Also, for at least three references the sleeve thickness was only $0.5 \mathrm{~mm}[53,54,58]$ and in one paper a nylon sleeve of $0.3 \mathrm{~mm}$ thickness was used [56]. These differences can make a small contribution to the spread in the data at the highest photon energies since it has been demonstrated that a $1 \mathrm{~mm}$ thick PMMA sleeve reduces the response of a Farmer type ionisation chamber by $0.2 \% \pm 0.1 \%$ in a $20 \mathrm{MV}$ beam [92]. On the other hand, the uncertainty on this sleeve perturbation is substantial and correcting certain data points for this would have only a minor influence. The effect of these differences was ignored in the further analysis. 
Recently, Krauss and Kapsch [72] reported experimental determined $k_{Q}$ values in two high energy photon beams, which were not incorporated in the final analysis presented here. The $k_{Q}$ values they reported in photon beams with $\mathrm{TPR}_{20,10}$ values of 0.716 and 0.762 were 0.9889 and 0.9743 , respectively, for the NE2571 and 0.9885 and 0.9759 for the NE2561, respectively.

Table 9: Experimental $k_{Q}$ values for the NE 2571 and the NE 2561 / NE 2611A chamber from the literature.

\begin{tabular}{|c|c|c|c|c|c|c|}
\hline Source reference & $\mathrm{TPR}_{20,10}$ & $\% d d(10)_{\mathrm{x}}$ & $\begin{array}{c}k_{Q} \\
\text { NE } 2571\end{array}$ & $u(1 s d)$ & $\begin{array}{c}k_{Q} \\
\text { NE 2561 } \\
\text { /NE 2611A }\end{array}$ & $\mathrm{u}(1 \mathrm{sd})$ \\
\hline Shortt et al. (1992) [56] & $\begin{array}{l}0.7720 \\
0.7850\end{array}$ & & $\begin{array}{l}0.9715 \\
0.9660\end{array}$ & $\begin{array}{l}0.0098 \\
0.0097\end{array}$ & & \\
\hline Boutillon et al. (1994) [57] & $\begin{array}{l}0.7110 \\
0.7250 \\
0.7640 \\
0.7720\end{array}$ & & & & $\begin{array}{l}0.9924 \\
0.9919 \\
0.9827 \\
0.9725\end{array}$ & $\begin{array}{l}0.0100 \\
0.0100 \\
0.0099 \\
0.0098\end{array}$ \\
\hline Ross et al. (1994) [54] & $\begin{array}{l}0.7580 \\
0.7800 \\
0.7910 \\
0.7950 \\
0.8150 \\
0.8210\end{array}$ & $\begin{array}{l}82.70 \\
86.60 \\
85.20 \\
90.10 \\
89.60 \\
92.30\end{array}$ & $\begin{array}{l}0.9729 \\
0.9628 \\
0.9672 \\
0.9577 \\
0.9602 \\
0.9570\end{array}$ & $\begin{array}{l}0.0039 \\
0.0039 \\
0.0039 \\
0.0039 \\
0.0039 \\
0.0039\end{array}$ & & \\
\hline Guerra et al. (1995) [58] & $\begin{array}{l}0.6730 \\
0.7570\end{array}$ & $\begin{array}{l}67.00 \\
75.90\end{array}$ & $\begin{array}{l}0.9946 \\
0.9769\end{array}$ & $\begin{array}{l}0.0065 \\
0.0064\end{array}$ & $\begin{array}{l}0.9956 \\
0.9759\end{array}$ & $\begin{array}{l}0.0065 \\
0.0064\end{array}$ \\
\hline $\begin{array}{l}\text { Palmans et al. (1999, 2002) [53, 59] } \\
\text { Palmans (1999) [49] }\end{array}$ & $\begin{array}{l}0.6780 \\
0.7440\end{array}$ & $\begin{array}{l}65.60 \\
74.20\end{array}$ & $\begin{array}{l}0.9954 \\
0.9786\end{array}$ & $\begin{array}{l}0.0050 \\
0.0052\end{array}$ & $\begin{array}{l}0.9882 \\
0.9729\end{array}$ & $\begin{array}{l}0.0061 \\
0.0071\end{array}$ \\
\hline Seuntjens et al. (2000) [55] & $\begin{array}{l}0.6820 \\
0.7580 \\
0.7940\end{array}$ & $\begin{array}{l}69.60 \\
80.50 \\
88.40\end{array}$ & $\begin{array}{l}0.9904 \\
0.9723 \\
0.9557\end{array}$ & $\begin{array}{l}0.0038 \\
0.0036 \\
0.0036\end{array}$ & $\begin{array}{l}0.9944 \\
0.9724 \\
0.9560\end{array}$ & $\begin{array}{l}0.0038 \\
0.0036 \\
0.0036\end{array}$ \\
\hline $\begin{array}{l}\text { LNHB data } \\
\left(\mathrm{TRP}_{20,10} \text { and } k_{Q}\right) \\
\text { from Andreo 2000) [60] } \\
\text { (\%dd10x from Ross 2005) [61] } \\
\text { (corrected for difference Fricke } \\
\text { correction Andreo and Seuntjens) [55] }\end{array}$ & $\begin{array}{l}0.6750 \\
0.7170 \\
0.7430 \\
0.7490 \\
0.7650 \\
0.7670 \\
0.7840 \\
0.7850\end{array}$ & $\begin{array}{l}67.36 \\
71.63 \\
75.02 \\
75.63 \\
78.43 \\
78.75 \\
81.96 \\
82.15\end{array}$ & $\begin{array}{l}0.9920 \\
0.9880 \\
0.9810 \\
0.9810 \\
0.9740 \\
0.9730 \\
0.9710 \\
0.9680\end{array}$ & $\begin{array}{l}0.0070 \\
0.0079 \\
0.0089 \\
0.0089 \\
0.0098 \\
0.0098 \\
0.0098 \\
0.0097\end{array}$ & & \\
\hline Palm et al. (2002) [51] & 0.6210 & 63.00 & 0.9940 & 0.0070 & & \\
\hline
\end{tabular}




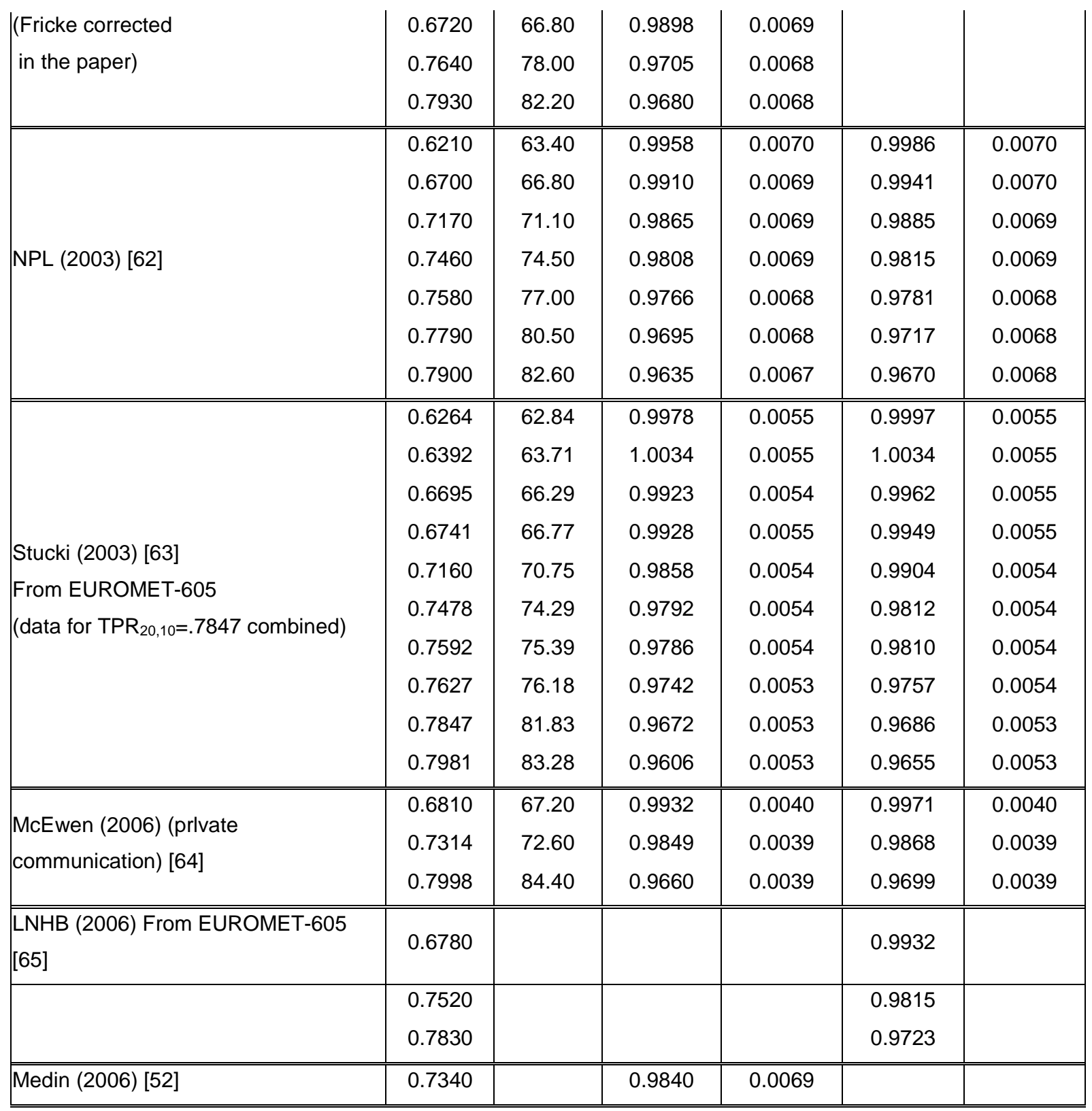

\section{A.4.5.2 Analysis of the NCS data together with the data from the literature}

The data measured by NCS, presented in Table 8, were added to this collection of data and as a function of $\mathrm{TPR}_{20,10}$ sigmoidal fits were made of the form:

$$
k_{Q}=A+\frac{B}{1+\exp \left(C \cdot\left(T P R_{20,10}-X_{0}\right)\right)}
$$

where $A, B, C$ and $X_{0}$ are fit parameters. 
The justification for this is that such fits give an excellent agreement with the stopping power ratios from Andreo (1994) [73] that form at the basis of the calculated $k_{Q}$ values in TRS-398. Also the second largest contribution to the beam quality dependence of $k_{Q}$ for a graphitewalled ionisation chamber, the wall correction factor $p_{\text {wall }}$, can be accurately modelled with such a function. Although third order polynomials are found to be accurate for these theoretical data as well, they have the disadvantage that for experimental data, modest fluctuations can give rise to substantial changes in shape of the curve. A sigmoid on the other hand will still preserve its smooth character.

The model is first normalised such that the value of $k_{Q}$ for a $T P R_{20,10}$ of 0.57 is unity. Here the assumption is made that for a high energy photon beam with a $\operatorname{TPR}_{20,10}$ similar to the one for ${ }^{60} \mathrm{Co} k_{\mathrm{Q}}$ is unity. An uncertainty is associated with this assumption. This results in the reduction of the number of free parameters in the model with one. In order to decide which of the four parameters to express in function of the other three, a fit to the NE 2571 data was performed and the parameter to which the fit is least sensitive is selected. It was found that this is parameter $\mathrm{B}$, leading to

$$
k_{Q}=A+(1-A) \cdot \frac{1+\exp \left(C \cdot\left(0.57-X_{0}\right)\right)}{1+\exp \left(C \cdot\left(\operatorname{TPR}_{20,10}-X_{0}\right)\right)}
$$

The parameters in the model are optimised with a least squares fitting algorithm (LevenbergMarquardt) $[70,71]$.

The $k_{Q}$ data as a function of $\% d d(10)_{\mathrm{x}}$ are fitted with a linear curve. The justification of this is similar as for the dependence as function of $\mathrm{TPR}_{20,10}$, i.e. that the stopping powers in the high energy x-ray beams are linearly dependent on \%dd(10) [69]. Some higher polynomial fits were tried to investigate if they lead to a better fit.

Figures 7 and 8 show all the data for the NE 2571 and the NE 2561/NE 2611A as a function of both beam quality specifiers together with the sigmoid and linear fits as described above. In these sigmoid fits $A$ was given the constant value of 0.80 . The reason for this will be explained below. Theoretical data from TRS-398, TG-51 and Rogers (1992) [20] are shown as well.

The root mean square of the deviation from both models and both ionisation chamber types is comparable (around $0.3 \%$ ). Performing quadratic and cubic polynomial fits to the data as a function of $\% d d(10)_{x}$ did not significantly alter this, so the linear model is deemed accurate enough. We conclude that there is no obvious preference of one beam quality specifier above another. It was decided to use $\mathrm{TPR}_{20,10}$ as beam quality specifier and the sigmoid fit is 
the best model we can find to represent the beam quality dependence of the data. The most important characteristic of the beam quality index $\mathrm{TPR}_{20,10}$ is its independence on the electron contamination in the incident beam. It is also a measure of the effective attenuation coefficient describing the approximately exponential decrease of a photon depth-dose curve beyond the depth of maximum dose. TPR 20,10 is obtained as a ratio of doses; it does not require the use of displacement correction factors at two depths when cylindrical chambers are used. For this reason, $\mathrm{TPR}_{20,10}$ is practically not affected by systematic errors in positioning the chamber at each depth, as the settings in the two positions will be affected in a similar manner. Finally, it is a parameter that is normally available through commissioning measurements and does not require special measurements using lead attenuators as it is the case for the \%dd(10)x parameter [67-69].

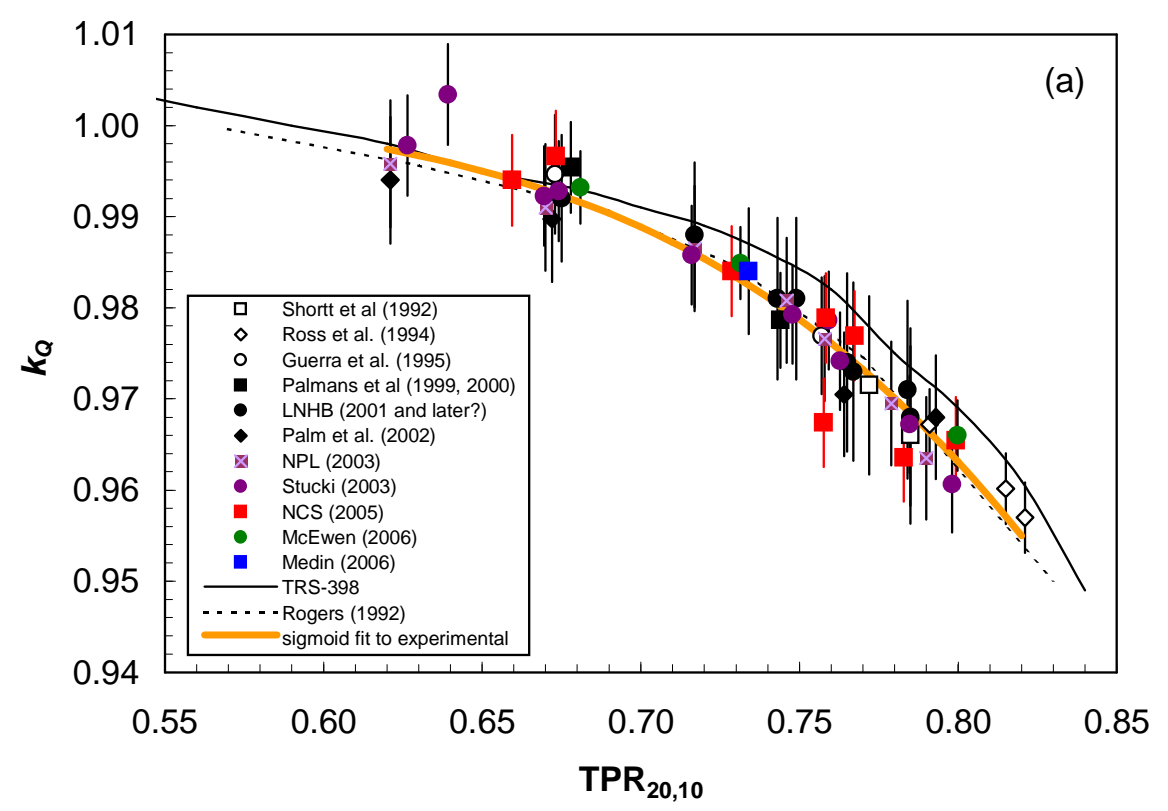




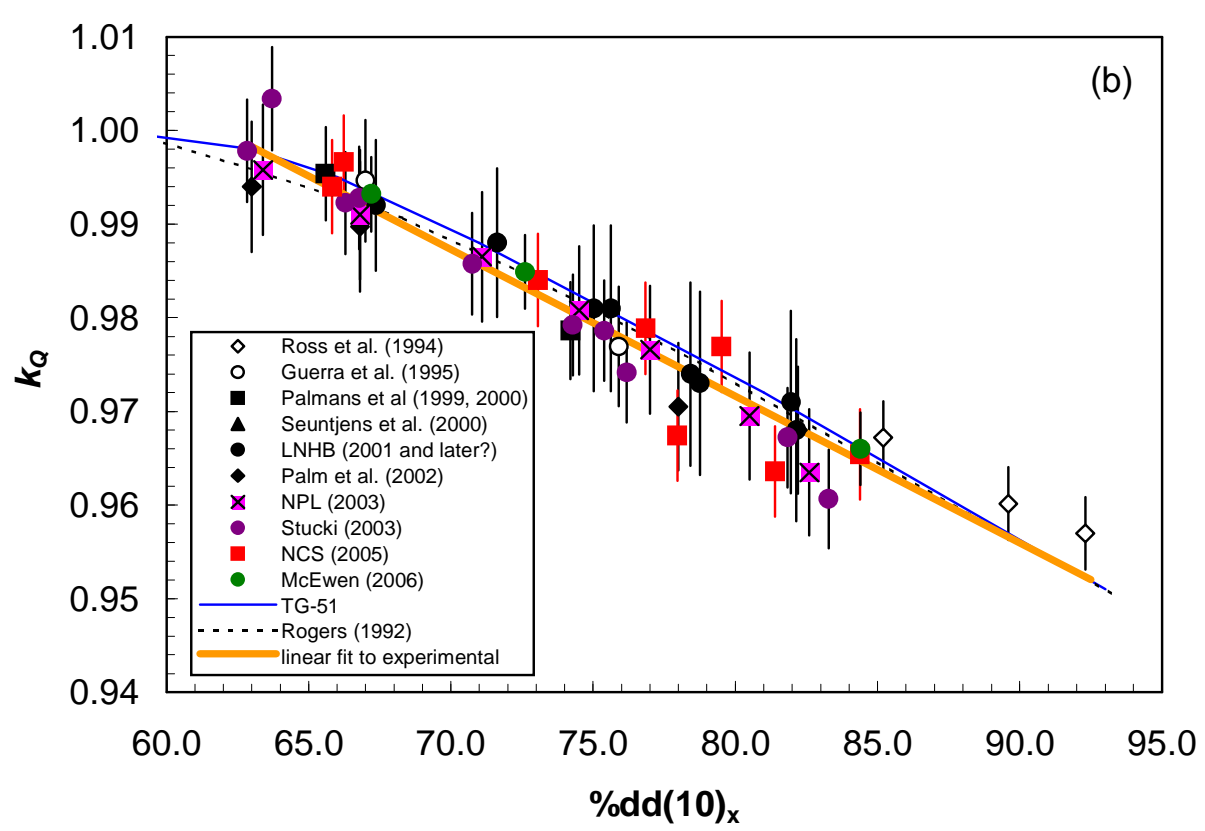

Figure 7: $k_{Q}$ data from the literature together with the data measured by NCS for the NE 2571 type ionisation chamber (a) as function of $\mathrm{TPR}_{20,10}$ with a sigmoid fit (with $\mathrm{A}=0.80)$ and $(\mathrm{b})$ as function of $\% d d(10)_{\times}$with a linear fit. 

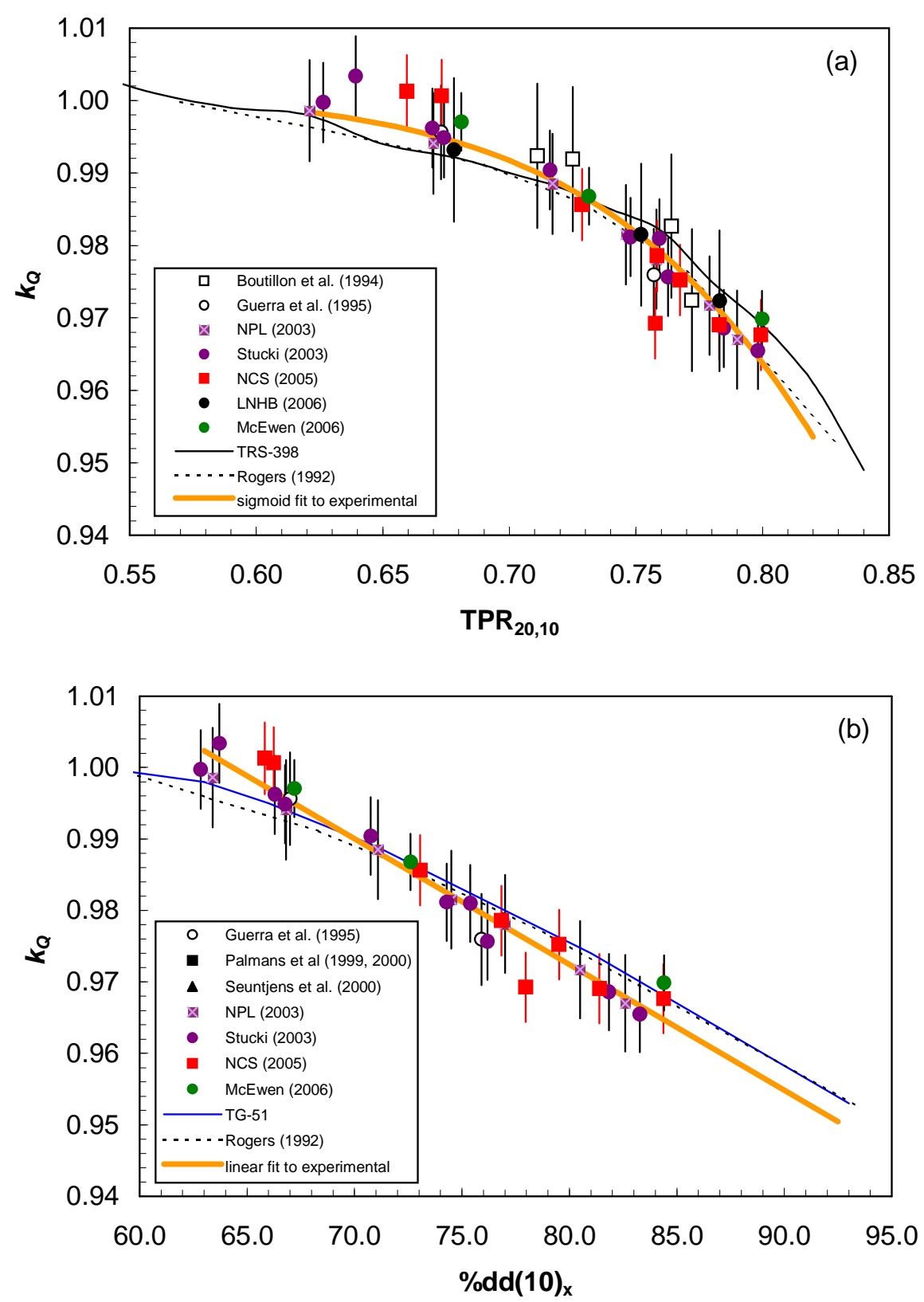

Figure 8: $k_{Q}$ data from the literature together with the data measured by NCS for the NE 2561 and NE 2611A type ionisation chamber (a) as function of $\operatorname{TPR}_{20,10}$ with a sigmoid fit (with $A=0.80$ ) and (b) as function of $\% d d(10)_{x}$ with a linear fit.

Figure 9 shows all the data points for the NE 2571 and the NE 2561/NE 2611A with various sigmoid fits in which none, one or two parameters are kept constant. Curves defining 1sd levels were calculated for the model by propagating through the model the $1 \mathrm{sd}$ values of the fit parameters obtained from the Levenberg-Marquardt algorithm [70, 71]. In addition an uncertainty of $0.02(1 \mathrm{sd})$ was assigned to the $\mathrm{TPR}_{20,10}$ value of 0.57 where $k_{Q}$ was assumed to be unity. This is consistent with the spread of TPR $R_{20,10}$ values in TRS-398 where $k_{Q}$ is unity 
for cylindrical ionisation chambers. It can be seen in Figure 9 that the contribution to the overall uncertainty is not large given that in the region around the $\mathrm{TPR}_{20,10}$ value of 0.57 the curve is fairly flat. The overall standard deviation is obviously energy dependent; it is small at $\mathrm{TPR}_{20,10}=0.57$ and grows as the beam quality increases.

It is obvious that leaving three parameters free leads to uncertainty estimates that are far too large, also the model tends to curve upward at the higher beam qualities which is unrealistic, compared with the theoretical beam quality dependence of the stopping powers. It was concluded that three free parameters in the model leaves too much degrees of freedom and the (remaining) parameter to which the model was least sensitive, which was found to be A, was kept constant. A range of values for $A$ between 0.5 and 0.9 were tried and it was found that at $A=0.80$ the standard deviation of the model was such that about $2 / 3$ data points fall within these 1sd curves. The tails of the distributions were less conform to a normal distribution but about $90 \%$ within the 2sd curves and about $95 \%$ within the 3sd curves for both the NE 2571 and NE 2561/NE 2611A. Hence it was decided that the sigmoid models with $A=0.80$ give a good representation of the experimental data with their experimental noise.

The lower graphs in Figure 9 show that keeping only one parameter free leads to an underestimation of the uncertainties.

The resulting values of the parameters $\mathrm{C}, \mathrm{A}$ and $\mathrm{X}_{0}$ in the sigmoid model are given in Table 10. The amount of digits given is sufficient to reproduce the fits presented above to within $0.01 \%$. The uncertainties on the parameters in Table 10 which were determined by the fit algorithm result in a relative standard uncertainty in the $k_{Q}$ values varying from $0.1 \%$ to $0.4 \%$. In order to enable the user a way of verifying the implementation of this parameterized model, the data are plotted in Figure 10.

The recent data of Krauss and Kapsch [72] are close (and within the uncertainties equal) to the values obtained with the parameterised model presented in this work. Therefore it is assumed that the addition of those data in a new analysis will not significantly affect the outcome of the fits.

Table 10: Fit parameters $X_{0}, C$ and $A$ in the sigmoid model resulting from this work

\begin{tabular}{|l|c|c|c|}
\hline \hline & $\mathrm{X}_{0}$ & $\mathrm{C}$ & $\mathrm{A}$ \\
\hline \hline NE 2571/ & & & \\
PTW 30012/ & $0.9198 \pm 0.0097$ & $11.67 \pm 0.92$ & 0.80 \\
Wellhöfer FC65G & & & \\
\hline NE 2561/NE 2611A & $0.8971 \pm 0.0125$ & $15.15 \pm 1.60$ & 0.80 \\
\hline
\end{tabular}



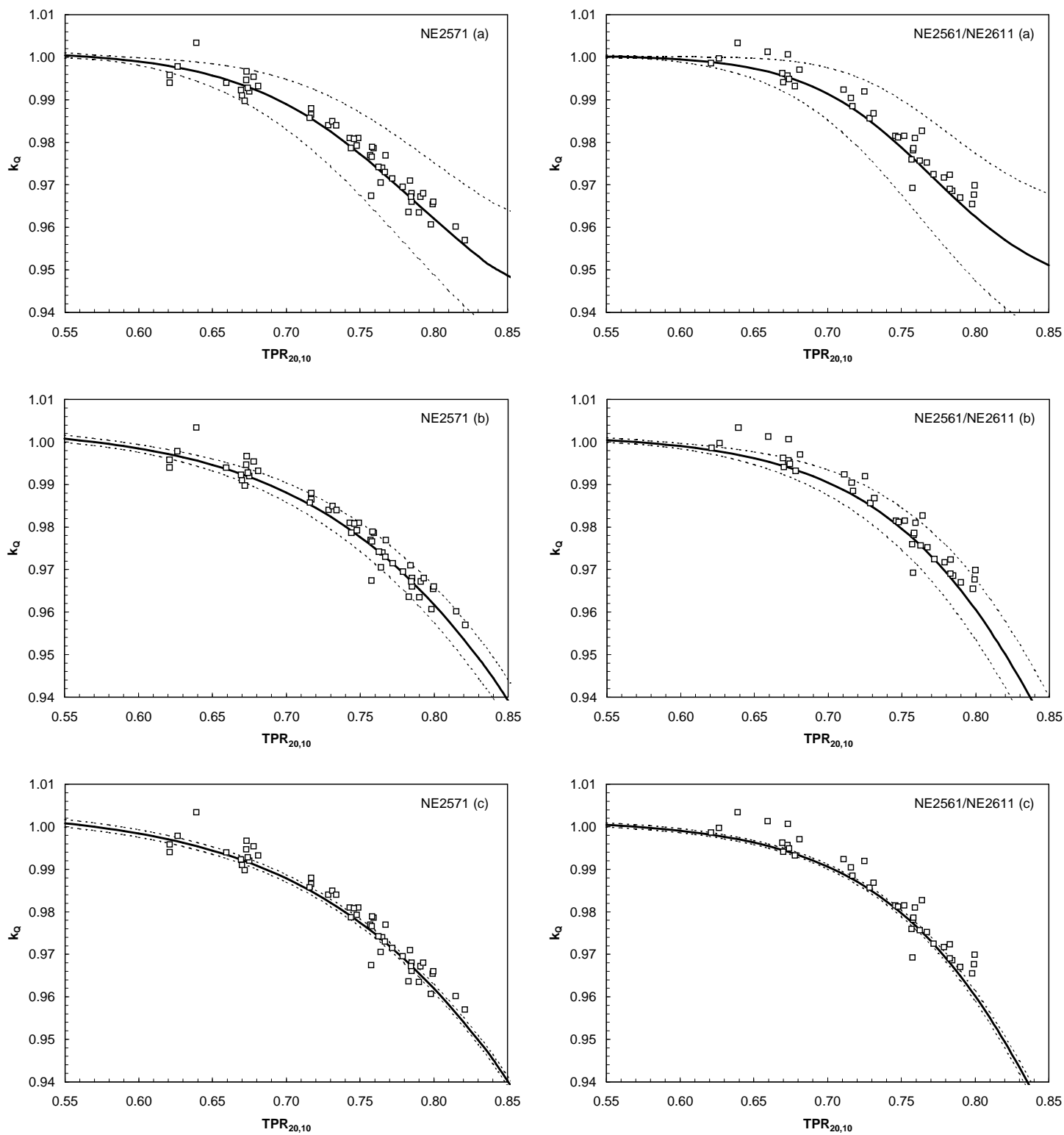

Figure 9: Sigmoid fit (solid lines) to all the data points (a) with $X_{0}, C$ and $A$ as free parameters, (b) with $X_{0}$ and $C$ as free parameters and $A=0.80$ and (c) with only $X_{0}$ as free parameter and $C$ and $A$ as in (b) for the NE 2571 (left) and for the NE 2561/NE 2611A (right). The dotted lines give the one standard deviations of the model for each fit. 


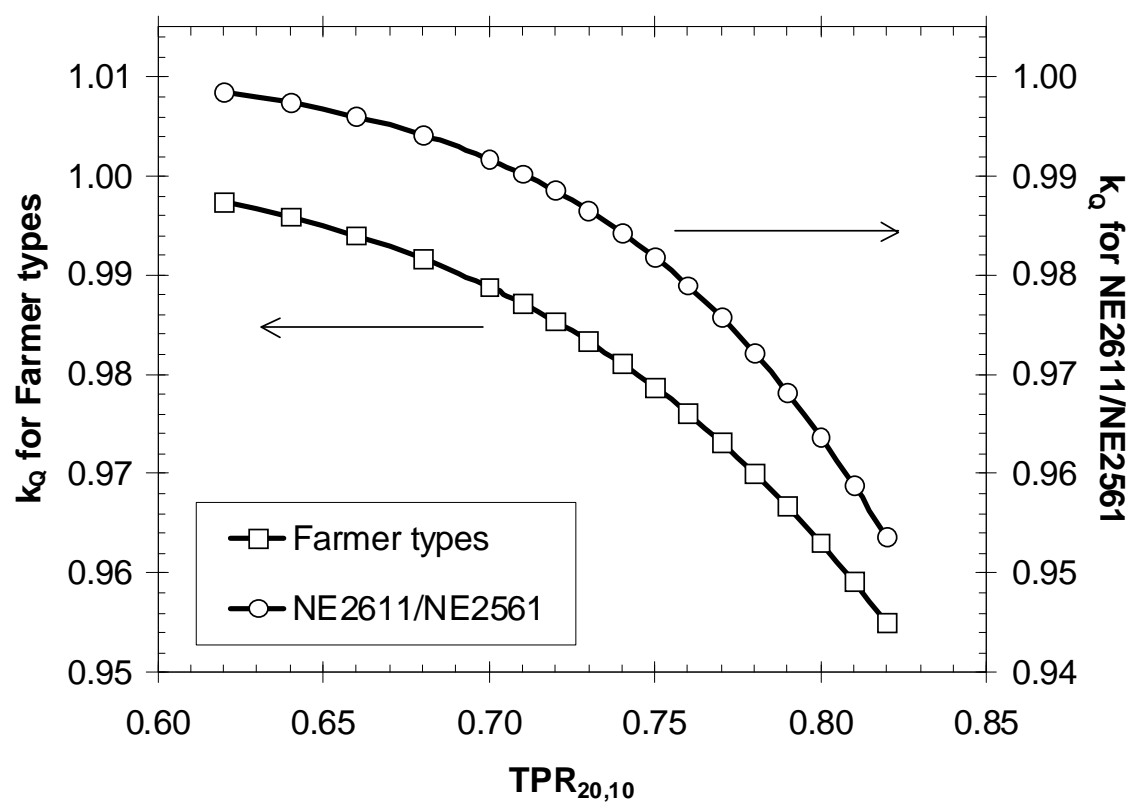

Figure 10: $k_{Q}$ data calculated according to equation (30) and the data of Table 10.

This plot is given for verification purposes.

\section{A.4.6 Limiting value for the expected uncertainty}

An estimate of the uncertainties in the calibration of a high energy photon beam is given in Table $11^{*}$. In order to determine the uncertainty on the absorbed dose to water in the user's beam by using a reference ionisation chamber, one must evaluate the uncertainties in the different physical quantities or procedures that contribute to the dose determination. These can be divided into two steps. Step 1 considers uncertainties up to the calibration of the reference dosimeter in terms of $N_{D, w}$ at the primary standard dosimetry laboratory. Step 2 deals with the calibration of the user's beam and includes the uncertainties associated with the measurements at the reference point in a water phantom. Step 2 also includes the uncertainty of the $k_{Q}$ value. A more detailed evaluation of the uncertainty contribution, due to $k_{Q}$ with the use of the sigmoid fit, is described in appendix A.4, section A.4.5.2.

\footnotetext{
* The uncertainty estimates given in Table 11 should be considered typical values; these values may vary depending on the expanded standard uncertainty quoted by Standards Laboratories for calibration coefficients and on the experimental uncertainty determined at the user's institution.
} 
Table 11: Estimated combined standard uncertainty on $D_{W, Q}$ at the reference depth in water for a high energy photon beam.

\section{Step 1: Primary Standard Dosimetry Laboratory \\ (NMI, LSDG)}

Type of physical quantity or procedure

$N_{D, w}$ calibration of the secondary (local) standard at PSDL

Long term stability of the secondary standard

Combined uncertainty in Step 1
Relative Standard

Uncertainty (\%)

0.6

\author{
Step 2: User beam \\ Type of physical quantity or procedure \\ Dosimeter reading $M_{\mathrm{corr}, Q}$ \\ (incl. influence quantities corrections $\prod k_{i}$ ) \\ Experimental procedure and stability of the dosimetry system 0.5 \\ Beam quality correction, $k_{Q}$ (based on NCS and literature data) $\quad 0.4$ \\ $\begin{array}{ll}\text { Combined uncertainty in Step } 2 & 0.8\end{array}$ \\ Combined standard uncertainty in $D_{w, Q}($ Steps $1+2)$
}

\title{
A.5 $k_{\mathrm{Q}}$ values and data for clinical high energy electron beams
}

For electron beams few experimental $k_{Q}$ or $k_{Q, Q \text { cross }}$ data are available in the literature. Guerra et al. [58] measured $k_{Q}$ in a $14 \mathrm{MeV}$ electron beam with a beam quality of $R_{50}=5.27 \mathrm{~cm}$. The measurement was however performed at the depth of maximum dose $z_{\max }=2.8 \mathrm{~cm}$, which used to be the recommended reference depth in earlier codes of practice. With this NCS protocol the measurement should have been performed at a depth of $z_{\text {ref }}=3.06 \mathrm{~cm}$. For the NE 2571 and NE 2561 they found $k_{Q}$ to be 0.906 and 0.904 respectively. Experimental $k_{Q}$ data-sets for plane-parallel chambers were reported by Stewart and Seuntjens [75] and by Stucki et al. [63] but in both cases no final published values are available. NPL has a substantial set of $k_{Q, Q c r o s s}$ data for plane-parallel chambers. However, they are measured using graphite calorimetry and the conversion procedure from absorbed dose to graphite to absorbed dose to water introduces a substantial uncertainty in these values. Furthermore, this conversion procedure uses NACP02 plane-parallel ionisation chambers as transfer instrument assuming that wall and cavity perturbation factors in electron beams both in graphite and in water are unity for this chamber types. This assumption is likely to be invalid as shown in a number of recent papers by Verhaegen et al, Buckley and Rogers, and McEwen et al. [76-78]. 


\section{A.5.1 Calculation of $k_{Q}$ and $k_{Q, Q c r o s s}$}

As shown before, $k_{\mathrm{Q}}$ and $k_{Q, Q c r o s s}$ are per definition ratios of calibration coefficients in terms of absorbed dose to water in two beam qualities:

$$
k_{Q}=\frac{N_{D, w, Q}}{N_{D, w,}{ }^{60} C_{C o}} \text { and } k_{Q, Q_{C r o s s}}=\frac{N_{D, w, Q}}{N_{D, w, Q_{C r o s s}}}
$$

where the beam quality index ${ }^{60} \mathrm{Co}$ is shown explicitly even though it is omitted in the rest of this report. This notation does not conform to the CoP but is used here for clarity to stress that $k_{Q}$ concerns a ratio of calibration coefficients in an electron and a photon beam.

Both these relations may be further generalized to:

$$
k_{Q, Q_{0}}=\frac{N_{D, w, Q}}{N_{D, w, Q_{0}}}=\frac{\frac{D_{w, Q}}{M_{Q, \text { corr }}}}{\frac{D_{w, Q_{0}}}{M_{Q_{0}, \text { corr }}}}
$$

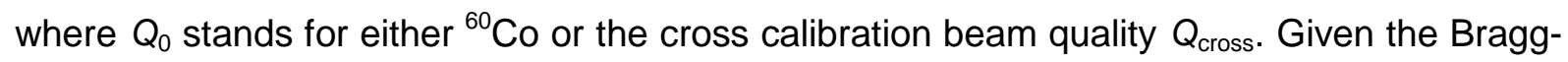
Gray relation between absorbed dose to water and absorbed dose to air in the ionisation chamber's air cavity:

$$
k_{Q, Q_{0}}=\frac{\frac{D_{\text {air }, Q}}{M_{Q, \text { corr }}}\left(s_{w, \text { air }}\right)_{Q} p_{Q}}{\frac{D_{\text {air }, Q_{0}}}{M_{Q_{0}, \text { corr }}}\left(s_{w, \text { air }}\right)_{Q_{0}} p_{Q_{0}}}=\frac{\frac{\left(w_{\text {air }} / e\right)_{Q}}{m_{\text {air }}}\left(s_{w, \text { air }}\right)_{Q} p_{Q}}{\frac{\left(W_{\text {air }} / e\right)_{Q_{0}}}{m_{\text {air }}}\left(s_{w, \text { air }}\right)_{Q_{0}} p_{Q_{0}}} \approx \frac{\left(s_{w, \text { air }}\right)_{Q} p_{Q}}{\left(s_{w, \text { air }}\right)_{Q_{0}} p_{Q_{0}}}
$$

since the mean energy required to produce an ion pair in dry air, $W_{\text {air }}$, is assumed to be independent of energy in photon and electron beams. $\left(s_{w, \text { air }}\right)_{Q}$ in equation (33) is the SpencerAttix water to air collision mass stopping power ratio for the beam quality $Q$ and the overall perturbation correction factor $p_{Q}$ conventionally consists of four contributions:

$$
p_{Q}=\left(p_{\text {dis }} p_{\text {cav }} p_{\text {wall }} p_{\text {cel }}\right)_{Q}
$$


where $p_{\text {dis }}$ corrects for the difference in ionisation at the effective point of measurement and the depth at which the absorbed dose is stated, $p_{\text {cav }}$ is a factor to correct for the response of an ionisation chamber for deviations from Bragg-Gray conditions due to the effect of the air cavity in the medium, $p_{\text {wall }}$ corrects for the difference in composition between the ionisation chamber wall and water and $p_{\text {cel }}$ corrects for the presence of the central electrode.

For stopping power data for ${ }^{60} \mathrm{Co}$ and for clinical electron beams there is no new information available since the publication of TRS-398. For the ${ }^{60} \mathrm{Co}$ calibration beam $s_{w \text {,air }}=1.133$ and for electron beams the data are taken from Burns et al. [31], i.e. at the measurement depth $z_{\text {ref }}\left(=0.6 R_{50, \text { dos }}-0.1 \mathrm{~cm}\right)$ the water to air mass stopping power ratio as a function of the beam quality $R_{50 \text {,dos }}$ (in $\mathrm{cm}$ ) is given by:

$$
s_{w, \text { air }}=1.253-0.1487 \cdot\left(R_{50, \text { dos }}\right)^{0.214}
$$

The choice of $R_{50 \text {,dos }}$ as the beam quality index is related to these considerations on the stopping power ratios. This choice is justified since it results in a simplification of the procedures in NCS-5 involving the derivation of the mean energy at the phantom surface based on $R_{50 \text {,dos }}$ as an intermediate step. It also directly relates the beam quality specifier to the penetration characteristics of the electron beam. The definition of $R_{50 \text {,dos }}$ is made in terms of absorbed dose levels, whereas usually ionisation curves are measured. Contrary to the situation in high energy photon beams, the variation of the water/air stopping-power ratios with depth is not negligible. Therefore, strictly, the depth ionisation curve should be converted to a depth dose curve using an accelerator dependent water-to-air stopping-power function of depth as was done in NCS-5. However generic expressions to derive $R_{50 \text {,dos }}$ from $R_{50 \text {,ion }}$ for a large number of accelerator types has proven to be sufficient.

\section{A.5.1.1 Farmer-type chambers}

For the perturbation correction factors for Farmer type chambers there is no new data available since the publication of TRS-398. Hence, for Farmer type chambers $k_{Q}$ is calculated as in equation (33) using data taken from TRS-398. Differences between the three Farmer type chambers were found to be negligible. The product $\left(s_{w, \text { air }}\right)_{60}{ }_{C o} p_{60}{ }_{C o}=1.102$. The only perturbations that are considered in the electron beam are $p_{\text {cav }}$ and $p_{\text {cel. }} p_{\text {cav }}$ depends on the beam quality and the cylindrical cavity radius $r_{\text {cyl }}$ (in $\mathrm{mm}$ ) as [87]: 


$$
p_{\text {cav }}=1-0.0217 \cdot r_{c y l} \cdot e^{-0.153 \cdot R 50, d o s}
$$

A mean radius of $3.15 \mathrm{~mm}$ was assumed for the three Farmer type chambers.

For $p_{\text {cel }}$ a uniform value of 0.998 was taken independent of electron beam quality [88].

The displacement correction factor $p_{\text {dis }}$ is inherently accounted for by using the concept of an effective point of measurement (see section 3.4). $p_{\text {wall }}$ is assumed to be unity for Farmer type chambers.

The $k_{\mathrm{Q}}$ values thus obtained for the Farmer type chambers can be summarized into a single formula of the form:

$$
k_{Q}=A-B \cdot\left[R_{50, \text { dos }}(Q)\right]^{C}
$$

The rationale behind this expression is that the underlying water to air mass stopping powers follow such a relationship (see equation 35 ). The parameters $\mathrm{A}, \mathrm{B}$ and $\mathrm{C}$ for calculating $k_{\mathrm{Q}}$ with this expression for the Farmer type chambers are given in Table 13. The maximum deviation between the calculated values for $k_{Q}$ and the values obtained with equation 37 is $0.05 \%$ in the beam quality index range from 4 to $20 \mathrm{~cm}$.

\section{A.5.1.2 Plane-parallel chambers}

For plane-parallel chambers only $p_{\text {cav }}$ and $p_{\text {wall }}$ play a role, under the assumption that qradient corrections are taken care of adequately by putting the effective point of measurement at the reference depth, hence:

$$
k_{Q, Q_{\text {cross }}}=\frac{\left(s_{w, \text { air }}\right)_{Q}}{\left(s_{w, \text { air }}\right)_{Q_{\text {cross }}}} \cdot \frac{\left(p_{\text {cav }} p_{\text {wall }}\right)_{Q}}{\left(p_{\text {cav }} p_{\text {wall }}\right)_{Q_{\text {cross }}}}
$$

For well-guarded plane-parallel chambers, such as the one recommended in this report, both TRS-398 and TG-51 assume that both perturbations factors are unity. Regarding $p_{\text {cav }}$, various papers indicate that it is constant as a function of energy (Ma et al. [79], Verhaegen et al. [76]) for well-guarded chambers. So even if the value is non-unity it would not influence the $k_{Q, Q \text { cross }}$ values since only ratios of $p_{\text {cav }}$ are needed. Regarding $p_{\text {wall }}$, however, several recent papers have demonstrated a variation of $p_{\text {wall }}$ at the reference depth with beam quality 
(Sempau et al. [80], Verhaegen et al. [76], Buckley and Rogers [77] and McEwen et al. [78]). Significant is that two different Monte Carlo codes were used (PENELOPE by Sempau et al. [80] and EGSnrc by Verhaegen et al. [76] and Buckley and Rogers [77]) and one semiempirical model (by McEwen et al. [78]). All of them predict a similar tendency of $p_{\text {wall }}$ values slightly above unity at low electron energies and increasing with decreasing energy. It was decided to estimate improved $p_{\text {wall }}$ values from these data.
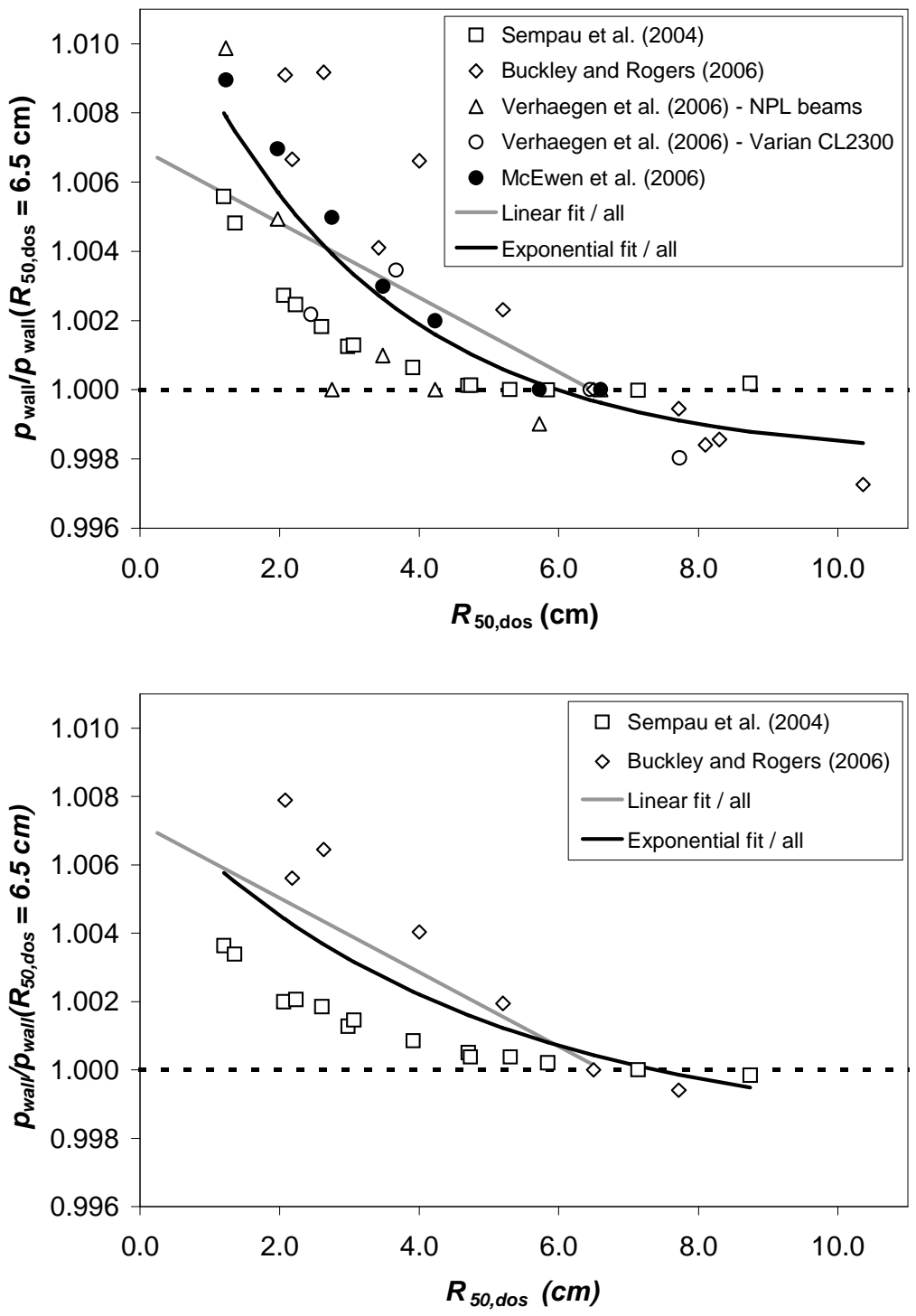

Figure 11: $p_{\text {wall }}$ values (relative to the values at $R_{50, \text { dos }}=6.5 \mathrm{~cm}$ ) obtained from Monte Carlo simulations and semi-empirical model calculations for the NACP02 and the Roos chambers. Linear and exponential fits are shown. 
Figure 11 shows these $p_{\text {wall }}$ values (relative to the values at $R_{50, \text { dos }}=6.5 \mathrm{~cm}$ ) for the NACP02 and the Roos chambers. The normalization at $R_{50, \mathrm{dos}}=6.5 \mathrm{~cm}$ is a bit arbitrary but is not important for $k_{Q, Q c r o s s}$ since again only ratios will be considered. Linear fits to all the data are shown as well as exponential fits of the form:

$$
p_{\text {wall }}\left(z_{\text {ref }}\right)=a+b \cdot e^{c \cdot R_{50, d o s}}
$$

Note that the fit parameters $a, b$ and $c$ are constant. It is obvious that there is considerable spread on the data points, possibly reflecting the variability of the $p_{\text {wall }}$ values for beams of varying type, and that the differences between the various fit models are not significant. The exponential fits were used for the further evaluation of $k_{Q, Q c r o s s}$ data.

The $k_{Q, Q \text { cross }}$ values calculated in that way can again be represented by a single equation of the form:

$$
k_{Q, Q_{\text {cross }}}=\frac{A-B \cdot\left[R_{50, \text { dos }}(Q)\right]^{C}}{A-B \cdot\left[R_{50, \text { dos }}\left(Q_{\text {cross }}\right)\right]^{C}}
$$

The parameters $A, B$ and $C$ for calculating $k_{Q, Q c r o s s}$ with this expression for the NACP02 and Roos type chambers is given in Table 13. The maximum truncation error introduced by this approximation is $0.05 \%$ in the beam quality range from 1 to $20 \mathrm{~cm}$.

Although we emphasize once again that it is not recommended to use plane-parallel chambers with ${ }^{60} \mathrm{Co}$ calibration coefficients the option is left open in case an electron beam with sufficiently high energy to perform a cross-calibration is not available. In this case $k_{\mathrm{Q}}$ values for the plane-parallel chambers need to be calculated as follows:

$$
k_{Q}=\frac{\left(s_{w, \text { air }}\right)_{Q}}{\left(s_{\text {w, air }}\right)_{60}} \cdot \frac{\left(p_{\text {cav }} p_{\text {wall }}\right)_{Q}}{\left(p_{\text {cav }} p_{\text {wall }}\right)_{60}{ }_{C o}}
$$

The main problem here is that the perturbation factors for the plane-parallel chamber in the ${ }^{60} \mathrm{Co}$ calibration beam have a considerable uncertainty. Various papers in the literature have reported that these perturbation factors can vary by $3 \%$ for chambers of the same type so it is difficult to recommend accurate generic data per chamber type. Nevertheless, for NACP02 and Roos type chambers the variability of these reported perturbation factors is not so dramatic and since the publication of TRS-398 various investigations have resulted in more 
accurate data for $p_{\text {wall }}$ in ${ }^{60} \mathrm{Co}$ by comparing dosimetry using both calibration routes (cross calibration in a high energy electron beam or calibration in ${ }^{60} \mathrm{Co}$ ). These data are shown in Table 12.

Table 12: Experimental $p_{\text {wall }}$ values in ${ }^{60} \mathrm{Co}$ for the NACP02 and Roos plane-parallel chamber types recommended in this report as reported in the literature.

\begin{tabular}{|c|c|c|c|}
\hline & & \multicolumn{2}{|c|}{$\mathrm{p}_{\text {wall }}$} \\
\hline Reference & Based on & NACP02 & PTW Roos \\
\hline Ding et al. (1998) [81] & TG-51 & 1.026 & \\
\hline Palm et al. (2000) [82] & TRS-381 & 1.020 & 1.014 \\
\hline Dohm et al. (2001) [83] & DIN 6800-2 [90] & & 1.024 \\
\hline Dohm et al. (2001) 83] & TG-51 & & 1.020 \\
\hline Palm et al. (2002) [84] & TRS-381 & & $1.018^{(*)}$ \\
\hline Stewart and Seuntjens (2002) [85] & TG-51 & 1.020 & 1.015 \\
\hline Stewart and Seuntjens (2002) [85] & TRS-398 & 1.020 & 1.016 \\
\hline Christ et al. (2002) [86] & DIN 6800-2 [90] & & 1.024 \\
\hline Christ et al. (2002) [86] & TG-51 & & 1.020 \\
\hline Palmans et al. (2003) [17] & TRS-398 & 1.015 & \\
\hline Palmans et al. (2003) [17] & TG-51 & 1.016 & \\
\hline \multirow[t]{5}{*}{ Kapsch et al. (2007) [89] } & DIN 6800-2 [90] & & 1.020 \\
\hline & $\begin{array}{c}\text { TRS-398/TRS-381 } \\
\text { (average) }\end{array}$ & 1.018 & 1.016 \\
\hline & $\mathrm{u}(1 \mathrm{sd})$ & $0.3 \%$ & $0.2 \%$ \\
\hline & TG-51 (average) & 1.021 & 1.018 \\
\hline & $u(1 s d)$ & $0.5 \%$ & $0.3 \%$ \\
\hline
\end{tabular}

${ }^{(\star)}$ Data point for the Wellhöfer-PPC35 ionisation chamber which is assumed to be of the same design

One comment on these data is that, although all these authors report to have measured a value of $p_{\text {wall }}$ in ${ }^{60} \mathrm{Co}$ for the plane-parallel chamber, they have actually measured a ratio of overall perturbation factors in ${ }^{60} \mathrm{Co}$ and the high energy beam assuming that the perturbation factors for the cylindrical chamber are perfectly known. The latter explains why the values determined based on TRS-398 are different from the values based on TG-51 and DIN 68002 since different data for the cylindrical chambers are recommended in different protocols. 
Hence for consistency, in the further analysis the perturbation correction factor data from TRS-398 for cylindrical chambers have been used.

Also noteworthy is that recent Monte Carlo simulated values have been published for $p_{\text {wall }}$ by Mainegra-Hing et al. [91] who found values of 1.021 for the NACP02 chamber and 1.009 for the Roos chamber. Even though they may represent more accurate values for $p_{\text {wall }}$ itself, it was decided to adopt the values of Table 12 for consistency with experimental data on the ratio of overall perturbations as mentioned above. These data can then be used to come up with a data set of $k_{Q}$ values for the plane-parallel chambers consistent with the Farmer data and the $k_{Q, Q c r o s s}$ data. Equation (41) can be modified to:

$$
k_{Q}=\frac{\left(s_{w, \text { air }}\right)_{Q}}{\left(s_{w, \text { air }}\right)_{\text {Co }}} \cdot \frac{\left(p_{\text {cav }} p_{\text {wall }}\right)_{Q}}{\left(p_{\text {cav }} p_{\text {wall }}\right)_{\text {high }-e}} \cdot \frac{\left(p_{\text {cav }} p_{\text {wall }}\right)_{\text {high }-e}}{\left(p_{\text {cav }} p_{\text {wall }}\right)_{\text {co }}}
$$

where the first two terms have been discussed already before where the high-e quality index refers to the cross calibration beam quality. The third term in equation (42) equals the reciprocal of the values presented in Table 12.

The resulting $k_{Q}$ values can again be summarized into a single formula of the form of equation (37) with the coefficients given in Table 13. The maximum truncation error introduced by this approximation is $0.05 \%$ in the beam quality range from 1 to $20 \mathrm{~cm}$.

\section{A.5.2 Calculated $k_{Q}$ values for high energy electron beams}

The resulting coefficients to calculate $k_{Q}$ and $k_{Q, Q \text { cross }}$ with equations (37) and (40) are given in Table 13. In Figure 12 and Figure 13 the data for $k_{Q}$ and $k_{Q, Q c r o s s}$ as a function of $R_{50 \text {,dos }}$ are presented.

Table 13: coefficients to calculate $k_{Q}$ and $k_{Q, Q c r o s s}$ with equations (37) and (40).

\begin{tabular}{|l|c|c|c|}
\hline & A & B & C \\
\hline Farmer types & 0.9345 & 0.0057 & 0.7733 \\
\hline NACP02 & 1.1955 & 0.2274 & 0.1479 \\
\hline Roos types & 1.1376 & 0.1700 & 0.1835 \\
\hline
\end{tabular}




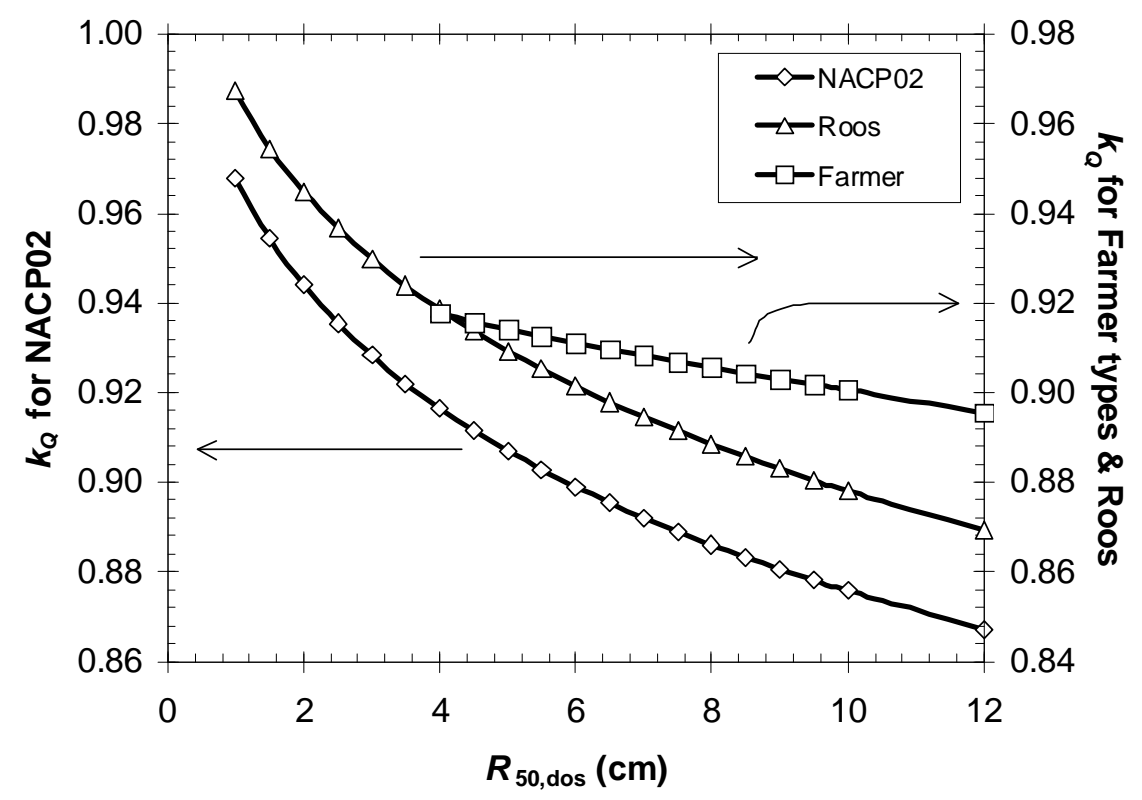

Figure 12: $k_{Q}$ data as a function of $R_{50 \text {,dos }}$ calculated according to equation (37) for the chamber types considered in this code of practice. This plot is given for verification purposes.
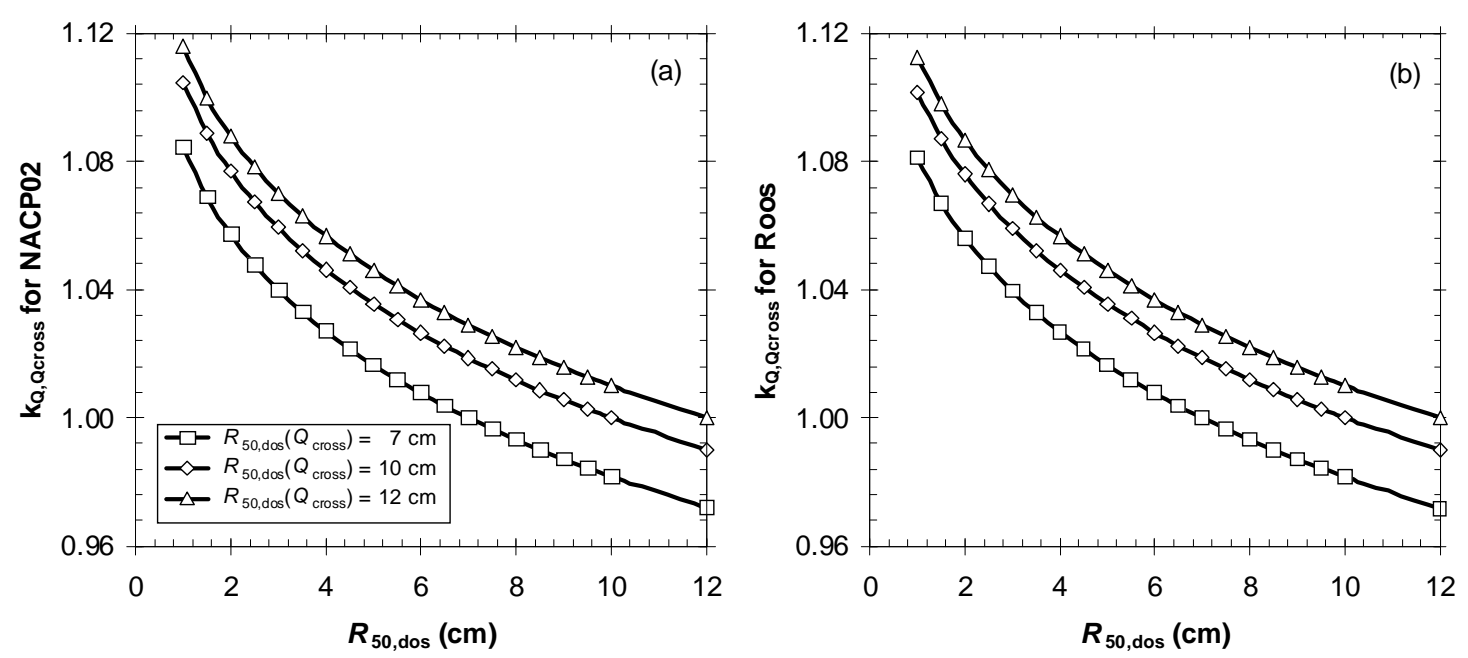

Figure 13: $k_{Q, Q \text { cross }}$ data as a function of $R_{50, \text { dos }}$ calculated according to equation (40) for (a) NACP02 chambers and (b) Roos chambers. These plots are given for verification purposes. 


\section{A.5.3 Comparison with experimental data}

Experimental data regarding $k_{Q}$ in electron beams are very scarce. Guerra et al. [58] reported an experimental $k_{Q}$ value of 0.904 for a NE 2571 in a $14 \mathrm{MeV}$ electron beam with an $R_{50}=$ $5.27 \mathrm{~cm}$. The present NCS report predicts a value of 0.914 for these conditions. This is a reasonable agreement given the difference in conditions (the measurement was done at the depth of dose maximum). Also the use of a different standard (Fricke) might influence the result.

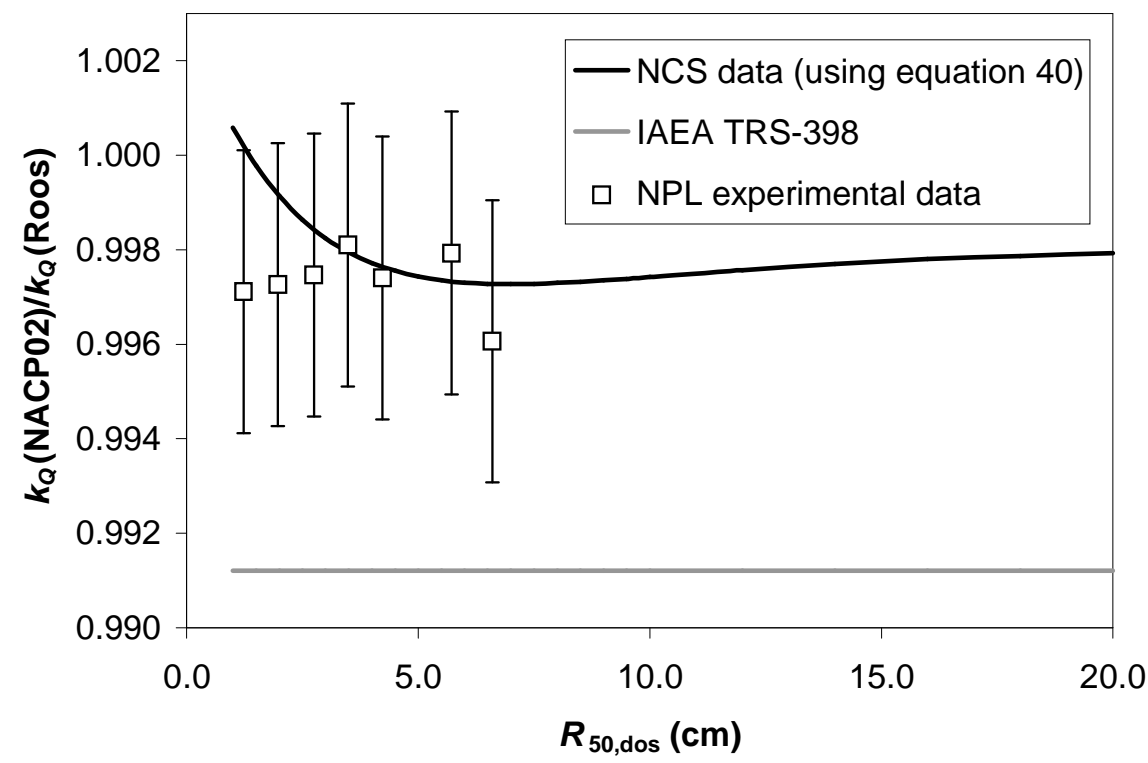

Figure 14: Ratio of $k_{\mathrm{Q}}$ data for the NACP02 and Roos type chambers compared with the experimental values from the NPL database.

NPL has a substantial set of calibration data for NACP02 and Roos type chambers. Even though there are at present question marks about the absolute calibration coefficients (the non-unity perturbation factors for the NACP02 mentioned before influence the conversion procedure from dose to graphite to dose to water, needed because the NPL calibrations are based on graphite calorimetry) the ratios of the $k_{Q}$ factors can be determined with a good accuracy. Figure 14 shows that the $k_{\mathrm{Q}}$ ratios between the NACP02 and Roos type chambers calculated in this report are in better agreement with the NPL data than the data from TRS-398. The curl-up effect in the NCS data shown in figure 14 is due to small differences 
between $\mathrm{p}_{\text {wall }}$ calculations for NACP02 and Roos chambers derived from the data presented in Figure 11.

\section{A.5.4 Limiting value for the expected uncertainty}

An estimate of the uncertainties in the calibration of a high energy electron beam is given in Tables 14,15 and $16^{*}$. In order to determine the uncertainty on the absorbed dose to water in the user's beam by using a reference ionisation chamber, one must evaluate the uncertainties in the different physical quantities or procedures that contribute to the dose determination. These can be divided into two or three contributions depending on the calibration procedure followed. Contribution one (Step 1 in Tables 14 and 16) considers uncertainties up to the calibration of the reference dosimeter in terms of $N_{D, w}$ at the standard dosimetry laboratory. Contribution two deals with either the absorbed dose determination in a electron beam with a Farmer type cylindrical ionisation chamber (Step 2 in Table 14) or the calibration of a plane-parallel chamber in the cross-calibration beam (Step 1 in Table 15), both including the uncertainties associated with the measurements at the reference point in a water phantom. Contribution three (Step 2 in Table 15 and 16) deals with the absorbed dose determination in a electron beam with a plane-parallel ionisation chamber and includes the uncertainties associated with the measurements at the reference point in a water phantom. Contributions two and three also include the uncertainty of the $k_{Q}$ and $k_{Q, Q c r o s s}$ values. A more detailed evaluation of the uncertainty budget, including the uncertainty on $k_{Q}$ and $k_{Q, Q c r o s s}$ is described in appendix $A 7$.

Although the estimated uncertainty on dosimetry using a plane-parallel ionisation chamber using a cross calibration is not much lower than using a ${ }^{60} \mathrm{Co}$ calibration beam, it has been demonstrated by various audits that the consistency of clinical reference dosimetry is substantially improved when implementing the cross-calibration procedure [33].

\footnotetext{
* The uncertainty estimates given in Tables 14,15 and 16 should be considered typical values; these values may vary depending on the expanded standard uncertainty quoted by Standards Laboratories for calibration coefficients and on the experimental uncertainty determined at the user's institution.
} 
Table 14: Estimated combined standard uncertainty on $D_{w, Q}$ measured with a Farmer type chamber at the reference depth in water for a high energy electron beam.

Step 1: Primary Standard Dosimetry Laboratory

Type of physical quantity or procedure

$N_{D, w}$ calibration of the secondary (local) standard at PSDL

Long term stability of the secondary standard

Combined uncertainty in Step 1
Relative Standard

Uncertainty (\%)

0.5

0.3

0.6

\section{Step 2: Farmer with ${ }^{60} \mathrm{Co}$ calibration coefficient in an electron beam}

with $\boldsymbol{R}_{\mathbf{5 0}}>\mathbf{4} \mathrm{cm}$

Type of physical quantity or procedure

Dosimeter reading (incl. influence quantities corrections $\prod k_{i}$ ), $M_{\text {corr, } Q} \quad 0.5$

Experimental procedure and stability of the dosimetry system 0.5

Beam quality correction, $k_{Q} \quad 1.2$

Combined uncertainty in Step 2

Combined standard uncertainty in $D_{w, Q}($ Steps $1+2)$ 
Table 15: Estimated combined standard uncertainty on $D_{w, Q}$ measured with a planeparallel ionisation chamber at the reference depth in water for a high energy electron beam based on a cross calibration in a high energy electron beam.

\section{Step 1: Cross calibration of plane-parallel chamber in a beam with Relative Standard \\ $R_{50}>7 \mathrm{~cm}$ \\ Uncertainty (\%)}

Type of physical quantity or procedure

Dose determination using the cylindrical chamber in a high energy

electron beam (from Table 14)

Dosimeter reading (incl. influence quantities corrections $\prod k_{i}$ ), $M_{\text {corr, } Q}$

Experimental procedure and stability of the dosimetry system

Combined uncertainty on $N_{D, w, Q c r o s s}$ in Step 1

Step 2: Plane-parallel chamber with cross-calibration coefficient in an electron beam

Type of physical quantity or procedure

Dosimeter reading (incl. influence quantities corrections $\Pi k_{i}$ ), $M_{\text {corr, } Q} \quad 0.5$

Experimental procedure and stability of the dosimetry system 0.5

Beam quality correction, $k_{Q, Q c r o s s} \quad 0.6$

Combined uncertainty in Step $2 \quad 0.9$

Combined standard uncertainty on $D_{w, Q}($ Steps $1+2)$

\footnotetext{
* The dosimeter reading in step 2 in Table 14 and the dosimeter reading in step 1 in table 15 are taken in the same beam quality and are therefore highly correlated. We assumed for the combined uncertainty contribution of these two dosimeter readings a value of $0.5 \%$
} 
Table 16: Estimated combined standard uncertainty on $D_{w, Q}$ measured with a planeparallel ionisation chamber at the reference depth in water for a high energy electron beam based on a ${ }^{60} \mathrm{Co}$ calibration coefficient in a PSDL.

\section{Step 1: Primary Standard Dosimetry Laboratory}

Type of physical quantity or procedure

$N_{D, w}$ calibration of the secondary (local) standard at PSDL

Long term stability of the secondary standard

Combined uncertainty in Step 1
Relative Standard

Uncertainty (\%)

0.5

0.3

0.6

\section{Step 2: Plane-parallel chamber with ${ }^{60} \mathrm{Co}$ calibration coefficient in} an electron beam

Type of physical quantity or procedure

Dosimeter reading (incl. influence quantities corrections $\prod k_{i}$ ), $M_{\text {corr, } Q} \quad 0.5$

Experimental procedure and stability of the dosimetry system 0.5

$\begin{array}{ll}\text { Beam quality correction, } k_{Q} & 1.7\end{array}$

Combined uncertainty in Step $2 \quad 1.8$

Combined standard uncertainty in $D_{w, Q}($ Steps $1+2)$

\section{A.5.5 Data for measurement in non-reference conditions}

The water to air mass stopping power ratios vary considerably with depth and in order to derive a depth dose curve from a measured depth ionisation curve with an ionisation chamber stopping power data as a function of beam quality and depth are needed. Burns et al. [31] derived from the same Monte Carlo simulations that led to equation (35) a more complicated equation for these data:

$$
s_{w, \text { air }}(z)=\frac{a+b \cdot \ln \left(R_{50}\right)+c \cdot\left[\ln \left(R_{50}\right)\right]^{2}+d \cdot \frac{z}{R_{50}}}{1+e \cdot \ln \left(R_{50}\right)+f \cdot\left[\ln \left(R_{50}\right)\right]^{2}+g \cdot\left[\ln \left(R_{50}\right)\right]^{3}+h \cdot \frac{z}{R_{50}}}
$$

with $a=1.075, b=-0.5087, c=0.0887, d=-0.084, e=-0.4281, f=0.0646, g=0.00309$ and $h=-0.125$.

Rogers [74] showed that for a wide range of accelerators these data are accurate to within $1.0 \%$ except at very shallow depths or depths beyond $1.1 \mathrm{z} / R_{50}$. 


\section{A.6 Expected differences with NCS-2 and NCS-5}

The ratio of dose to water values obtained using this protocol and the old protocols is given by:

$$
\frac{D_{w, Q}(N C S-18)}{D_{w, u}(N C S-2)}=\frac{N_{D, w}}{N_{K}} \times \frac{k_{Q}}{C_{w, u}(Q)}
$$

for photon beams and

$$
\frac{D_{w, Q}(N C S-18)}{D_{w, e}(N C S-5)}=\frac{N_{D, w, Q_{0}}}{N_{K}} \times \frac{k_{Q, Q_{0}}}{C_{w, e}(Q)}
$$

for electron beams, assuming that the corrected ionisation chamber readings are the same for both protocols. In equation (45) $k_{Q, Q_{0}}$ stands for either $k_{Q}$ or $k_{Q, Q c r o s s}$ depending on the calibration route and correspondingly $N_{D, w, Q o}$ stands for the absorbed dose to water calibration coefficient in either a cross calibration beam $Q_{\text {cross }}$ or a ${ }^{60} \mathrm{Co}$ photon beam.

It is clear that the ratio of dose to water values obtained using this protocol and the old protocols is determined by two components: the ratio of absorbed dose to water to air kerma calibration coefficients and the ratio of conversion factors. The former is dependent on the calibration laboratory and chamber type whereas the second is purely dependent on the data used for a particular chamber type.

The interpretation of these differences can be made easier by introducing a 'calculated' absorbed dose to water calibration coefficient $N_{D, w}^{*}$ and $k_{Q}$ values derived from the old protocols.

The 'calculated' absorbed dose to water calibration coefficient is calculated as:

$$
N_{D, w}^{*}=N_{K} \times C_{w, u}\left({ }^{60} \mathrm{Co}\right)
$$

and the $k_{\mathrm{Q}}$ derived from the old NCS protocols can be obtained by multiplying the numerator and denominator of expression (33) with the product $k_{\text {att }} k_{m} k_{\text {ce }}(1-g)$, which is entirely related to in-air perturbations during an air kerma calibration. For photon beams this gives: 


$$
k_{Q}(N C S-2)=\frac{\left(s_{w, a i r}\right)_{Q} p_{Q}}{\left(s_{w, a i r}\right)_{60}{ }_{C o} p_{60}{ }_{C o}} \times \frac{k_{a t t} k_{m} k_{c e}(1-g)}{k_{\text {att }} k_{m} k_{c e}(1-g)}=\frac{C_{w, u}(Q)}{C_{w, u}\left({ }^{60} C o\right)}
$$

and for a plane-parallel chamber in a electron beam $\left(k_{c e}=1\right)$ :

$$
k_{Q, Q_{\text {cross }}}(N C S-5)=\frac{\left(s_{w, \text { air }}\right)_{Q} p_{Q}}{\left(s_{w, \text { air }}\right)_{Q_{\text {cross }}} p_{Q_{\text {cross }}}} \times \frac{k_{\text {att }} k_{m}(1-g)}{k_{\text {att }} k_{m}(1-g)}=\frac{C_{w, e}(Q)}{C_{w, e}\left(Q_{\text {cross }}\right)}
$$

For photons, equation (44) can then be re-written as:

$$
\frac{D_{w, Q}(N C S-18)}{D_{w, u}(N C S-2)}=\frac{N_{D, w}}{N_{D, w}^{*}} \times \frac{k_{Q}(N C S-18)}{k_{Q}(N C S-2)}
$$

The expression is thus split in two factors. The first one is the ratio of a measured and a calculated absorbed dose to water calibration coefficient and its deviation from unity indicates an inconsistency between the theoretical data in the old protocol and the measured ratio of absorbed dose to water and air kerma calibration coefficients. This factor is independent of the data in the new code of practice. The second one reflects the change of chamber dependent data for the clinical beams in the new code compared to the old code. The ratio of absorbed dose to water values can thus entirely be studied in terms of calibration data for a particular chamber in the standard laboratory and data used in the protocol.

A similar expression can be written for electron dosimetry based on a calibration in ${ }^{60} \mathrm{Co}$ with a Farmer type chamber (or the unfavourable option of a plane-parallel chamber calibrated in $\left.{ }^{60} \mathrm{Co}\right)$ :

$$
\frac{D_{w, Q}(N C S-18)}{D_{w, e}(N C S-5)}=\frac{N_{D, w}}{N_{D, w}^{*}} \times \frac{k_{Q}(N C S-18)}{k_{Q}(N C S-5)}
$$

In this case the expression for $k_{Q}$ (NCS-5) is analogous to equation (47) using the data of the old electron code of practice (where for a plane-parallel chamber $k_{c e}$ equals 1 ).

For electron dosimetry using a plane-parallel chamber with a cross calibration, the differences are more complicated since the $N_{\mathrm{K}}$ and $N_{D, \mathrm{w}}$ calibration coefficients are for different chambers, but it can be easily shown, that: 


$$
\frac{D_{w, Q}(N C S-18)}{D_{w, e}(N C S-5)}=\frac{N_{D, w}^{\text {Farmer }}}{N_{K}^{p p}} \times \frac{M_{Q_{\text {cross }}}^{\text {Farmer }}}{M_{Q_{\text {cross }}^{p p}}^{p p}} \times \frac{k_{Q_{\text {cross }}}^{\text {Farmer }}}{C_{w, e}^{p p}\left(Q_{\text {cross }}\right)} \times \frac{k_{Q, Q_{\text {cross }}^{p p}}^{p p}(N C S-18)}{k_{Q, Q_{\text {cross }}^{p p}}^{p}(N C S-5)}
$$

so the ratio can again be assessed in terms of a ratio of calibration coefficients and protocol data, but in addition there is a ratio of two chamber readings in the clinical beam involved. The ratio is thus not solely due to measurements in the standards laboratory and protocol data but can be influenced by the local set-up in the clinical beam. This could for example lead to a different ratio being observed by different users with the same equipment in the same beam.

The expected differences based on these equations for the chambers recommended in this report are discussed below for photon and electron beams.

\section{A.6.1 High energy photon beams}

The data to evaluate equation (49) for high energy photon beams are given in Table 17. The ratio of the measured and the calculated calibration coefficient is a generic value derived from the paper by Palmans et al. (2002) [16]. Hence, the values in this example are indicative and the user who wants to know the change for his particular therapy unit and equipment should evaluate this ratio for each individual calibrated ionisation chamber. The ratio of the $k_{Q}$ data is also shown as a function of $\mathrm{TPR}_{20,10}$ in Figure 15. The two contributions to the difference are tabulated separately but since the ratio of the measured and the calculated calibration coefficient is very close to unity, it is easy to see that the overall differences are smaller than $1 \%$ in all cases.

Table 17: Components according to equation (49) of typical differences in absorbed dose to water in high energy photon beams measured with this code of practice and NCS-2.

(1) From Palmans et al. (2002) taking into account a $0.3 \%$ difference between the disseminated air kerma calibrations and the air kerma calibrations reported in that paper.

(2) For the PTW 30012 and the Wellhöfer-FC65G no data are given in NCS-2. The same data as for the NE 2571 were assumed.

\begin{tabular}{|l|l|l|l|l|}
\hline & NE 2571 & PTW 30012 & Wellhöfer FC65G & NE 2611A/NE 2561 \\
\hline PSDL & $\frac{N_{D, w}}{N_{D, w}^{*}}$ & & & \\
\hline LSDG $^{(1)}$ & 0.999 & 0.999 & 0.997 & \\
\hline
\end{tabular}




\begin{tabular}{|l|l|l|l|l|}
\hline TPR $_{20,10}$ & $\frac{k_{Q}(N C S-18)}{k_{Q}(\text { NCS }-2)}$ & & & \\
\hline 0.60 & 1.001 & & & \\
\hline 0.64 & 1.001 & 1.001 & 1.001 & 1.002 \\
\hline 0.68 & 1.001 & 1.001 & 1.001 & 1.004 \\
\hline 0.72 & 0.998 & 1.001 & 1.001 & 1.003 \\
\hline 0.75 & 0.999 & 0.998 & 0.998 & 1.002 \\
\hline 0.78 & 0.999 & 0.999 & 0.999 & 1.001 \\
\hline 0.81 & 1.000 & 0.999 & 0.999 & 0.999 \\
\hline
\end{tabular}

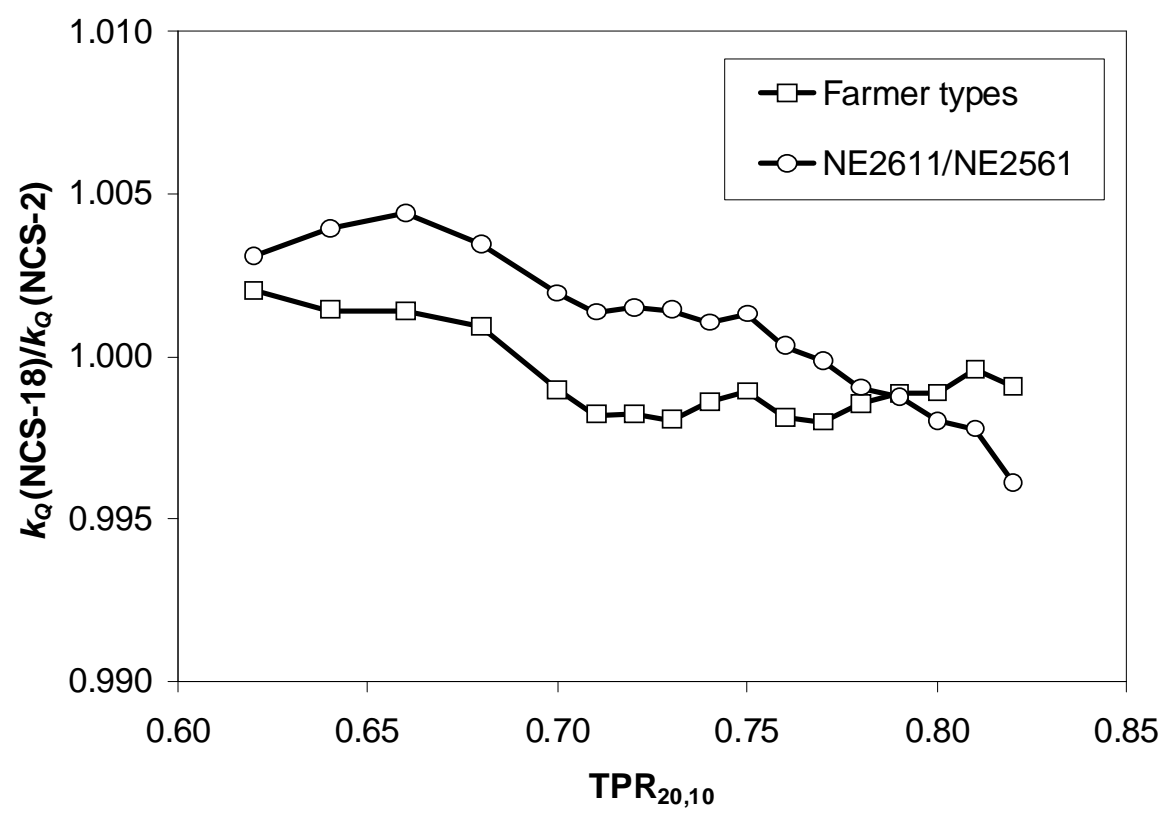

Figure 15: Ratio of $k_{Q}$ values for photon beams recommended in this protocol and derived from NCS-2.

\section{A.6.2 High energy electron beams}

The data to evaluate equation (50) for high energy electron beams are given in Table 18 as well as the overall resulting absorbed dose to water ratios obtained with the new protocol and NCS-5. Here, the ratio of the measured and the calculated calibration coefficient are taken from the paper by Palmans et al. (2003) [17] and thus, again, the values are indicative and should be investigated by the user for his particular set-up. For the NACP02 chamber the air kerma calibration coefficient $N_{K}$ was determined according the procedures in NCS-5, i.e. with a $3 \mathrm{~mm}$ graphite build-up plate. The ratios of $k_{Q}$ data as a function of beam quality are also shown graphically in Figure 16. One complication in such a comparison exercise is that the 
reference depths in both codes of practice can be different. This is in general the case for higher energies since the new reference depth can be deeper than the dose maximum. To make a generic comparison possible as an example, the $C_{\mathrm{w}, \mathrm{e}}$ values for NCS-5 were calculated at the new $z_{\text {ref }}$ (derived from $R_{50}$ ) according to the tables of NCS-5. In principle, measurements at both depths should be taken and the ionisations converted with the different water to air collision mass stopping powers and perturbation correction factors at both depths to allow a rigorous comparison of dosimetry using the two protocols. The presented values are thus only indicative and a proper comparison should be performed for each electron beam unit and dosimetry system. The differences for the NE 2571 and the NACP02 are in general smaller than $1 \%$ over the entire range of beam qualities. Differences would not be expected to be larger than $1 \%$ so any larger deviation could be an indication that further investigation of the source of the difference is needed. The differences observed in Table 18 are consistent with the findings of Palmans et al. (2003) [17] for a limited set of three electron beam energies.

Table 18: Components and total values according to equation (50) of typical differences in absorbed dose to water in high energy electron beams measured with this code of practice and NCS- 5 using a calibration coefficient in ${ }^{60} \mathrm{Co}$.

(1) From Palmans et al. (2003) taking into account a 0.3\% difference between the disseminated air kerma calibrations and the air kerma calibrations reported in that paper

(2) The newest $p_{\text {wall }}$ value for the NACP02 chamber in ${ }^{60} \mathrm{Co}$ (from this report) is used. This affects the ratio of $k_{Q}$ data, but also the ratio of measured and "calculated" calibration coefficients over an amount which is equal but which corrects in the opposite direction. The overall resulting absorbed dose to water ratios are thus unaffected by this choice.

\begin{tabular}{|l|l|l|l|l|}
\hline & NE 2571 & NACP02 & NE 2571 & NACP02 \\
\hline PSDL & $\frac{N_{D, w}}{N_{D, w}^{*}}$ & & & \\
\hline LSDG $^{(1)}$ & 0.999 & $0.994^{(2)}$ & & \\
\hline$R_{50}$ & $\frac{k_{Q}(N C S-18)}{k_{Q}(N C S-5)}$ & & $\frac{D_{w, Q}(N C S-18)}{D_{w, e}(N C S-5)}$ & \\
& $\mathrm{N} / \mathrm{A}$ & 1.011 & $\mathrm{~N} / \mathrm{A}$ & \\
\hline 1.0 & $\mathrm{~N} / \mathrm{A}$ & 1.005 & $\mathrm{~N} / \mathrm{A}$ & 1.005 \\
\hline 2.0 & $\mathrm{~N} / \mathrm{A}$ & 1.001 & $\mathrm{~N} / \mathrm{A}$ & 0.999 \\
\hline 3.0 & 0.995 & 1.000 & 0.994 & 0.995 \\
\hline 4.0 & 0.998 & 1.002 & 0.997 & 0.994 \\
\hline 5.0 & & & & 0.996 \\
\hline
\end{tabular}




\begin{tabular}{|l|l|l|l|l|}
\hline 7.5 & 1.005 & 1.001 & 1.004 & 0.995 \\
\hline 10.0 & 1.007 & 1.001 & 1.006 & 0.995 \\
\hline 12.0 & 1.004 & 1.001 & 1.003 & 0.995 \\
\hline
\end{tabular}

A specific example of the evaluation of equation (51) for high energy electron beams in the case of a cross calibration beam quality of $R_{50}=7 \mathrm{~cm}$ is given in Table 19 . The ratios of $k_{Q, Q c r o s s}$ data for this specific case as a function of beam quality are also shown graphically in Figure 16. The same remark as above on the different measurement depths in both codes, and thus on the non-generality of the data, applies here. This example is again based on the calibration data in Palmans et al. (2003) [17]. It must be noted that not only the measurement data can vary according to the conditions of the experiment. In addition, the ratio of $k_{Q, Q_{\text {Cross }}}^{p p}(N C S-18)$ and $k_{Q, Q_{\text {Cross }}^{p p}}(N C S-5)$ will show a slight dependence on the choice of the cross calibration beam quality since the data for the cross calibration beam are not the same in both protocols. For the beam quality range considered in this protocol, however, this variation was found to be smaller than $0.2 \%$. The differences for the NACP02 are again smaller than $1 \%$ over the entire range of beam qualities. Differences would not be expected to be larger than $1 \%$ and a larger deviation could be an indication that further investigation of the source of the difference is needed. The differences observed in Table 19 are consistent with the findings of Palmans et al. (2003) [17] for a limited set of three electron beam energies. 
Table 19: Components and total values according to equation (51) of typical differences in absorbed dose to water in high energy electron beams measured with this code of practice and NCS-5 using a plane-parallel chamber with a cross calibration coefficient.

\begin{tabular}{|c|c|c|c|}
\hline & NACP02 & & \\
\hline PSDL & $\frac{N_{D, w}^{\text {Farmer }}}{N_{K}^{p p}}$ & & \\
\hline \multirow[t]{2}{*}{ LSDG } & 0.3211 & & \\
\hline & $\frac{M_{Q_{\text {cross }}}^{\text {Farmer }}}{M_{Q_{\text {cross }}^{p p}}^{p p}}$ & $\frac{k_{Q_{\text {cross }}}^{\text {Farmer }}}{C_{w, e}^{p p}\left(Q_{\text {cross }}\right)}$ & $\frac{N_{D, w}^{\text {Farmer }}}{N_{K}^{p p}} \times \frac{M_{Q_{\text {cross }}^{\text {Farmer }}}^{\text {Fo }}}{M_{Q_{\text {cross }}^{p p}}^{p p}} \times \frac{k_{Q_{\text {cross }}^{\text {Farmer }}}^{\text {Fo }}}{C_{w, e}^{p p}\left(Q_{\text {cross }}\right)}$ \\
\hline$R_{50}\left(Q_{\text {cross }}\right) \approx 7 \mathrm{~cm}$ & 3.425 & 0.906 & 0.997 \\
\hline$R_{50}$ & & $\frac{\left.k_{Q, Q_{\text {cross }}^{p p}}^{p C S}-18\right)}{k_{Q, Q_{\text {Cross }}}^{p p}(N C S-5)}$ & $\frac{D_{w, Q}(N C S-18)}{D_{w, e}(N C S-5)}$ \\
\hline 1.0 & & 1.009 & 1.005 \\
\hline 2.0 & & 1.003 & 1.000 \\
\hline 3.0 & & 0.999 & 0.996 \\
\hline 4.0 & & 0.998 & 0.995 \\
\hline 5.0 & & 1.000 & 0.996 \\
\hline 7.5 & & 0.999 & 0.996 \\
\hline 10.0 & & 0.999 & 0.996 \\
\hline 12.0 & & 0.999 & 0.995 \\
\hline
\end{tabular}
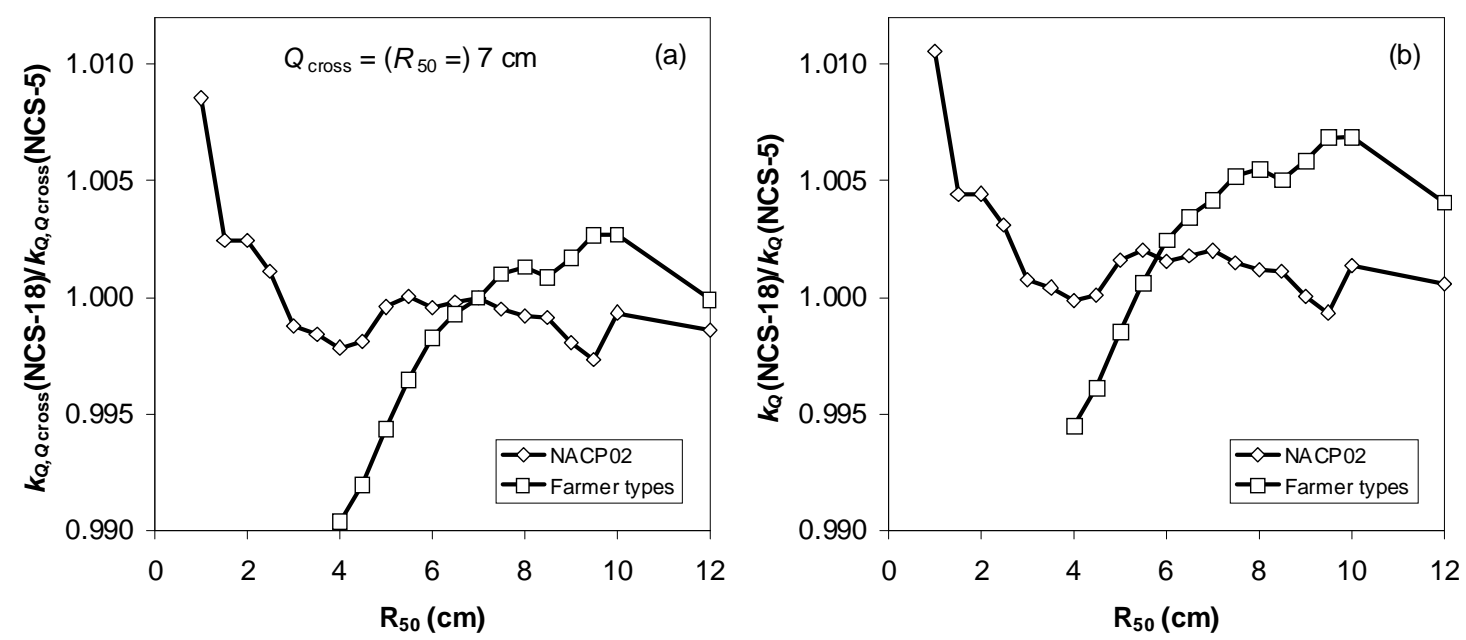

Figure 16:_Ratio of (a) $k_{Q, Q c r o s s}$ values (b) $k_{Q}$ values for electron beams recommended in this protocol and derived from NCS-5. 


\section{A.7 Expression of Measurement Uncertainty}

\section{A.7.1 Introduction}

It is recommended to base the estimation of measurement uncertainty on the guidelines presented in the ISO Guide to the Expression of Uncertainty in Measurement [93] and in document EA-4/02 of The European Co-operation for Accreditation [94]. The uncertainty budgets reported in this CoP follow the principles and analysis methods set out in these documents and the assessment of the uncertainties associated to clinical measurement results should be carried out in accordance with the recommendations of the ISO Guide and EA-4/02. This appendix provides an example of an uncertainty analysis for an absorbed dose to water determination in a clinical photon beam produced by a medical linear accelerator. Usually the uncertainty in an absorbed dose determination is dominated by a number of uncertainty contributions, while others have a small or negligible effect. For practical reasons a value for the smallest uncertainty is set, which has to be considered in the uncertainty analysis. Contributions below this value will be ignored. However, these contributions have to be evaluated and documented. In the example given below, the limiting value for an uncertainty contribution (relative standard uncertainty) to be considered is set to $0.1 \%$.

\section{A.7.2 General procedure of the uncertainty estimation}

The result of the $D_{w, Q}$ (output quantity) has to be calculated from a number of input quantities according to the equation:

$$
D_{w, Q}=M_{c o r r, Q} N_{D, w} k_{Q}
$$

where:

$D_{w, Q} \quad$ is the absorbed dose to water in the users beam $Q$ at the reference depth

$M_{c o r r, Q}$ the electrometer reading corrected for influence quantities, e.g. $k_{T P} k_{h} k_{\text {pol }} k_{s}$

$N_{D, w} \quad$ is the absorbed dose to water calibration factor in the ${ }^{60} \mathrm{Co}$ reference beam quality at $T_{\text {ref }}=20^{\circ} \mathrm{C}$ and $P_{\text {ref }}=101.325 \mathrm{kPa}$ and $50 \%$ relative humidity.

$k_{Q} \quad$ is the beam quality correction factor that depends on the chamber type and radiation beam quality of the users beam. 
The output quantity $D_{w, Q}$ is considered a function of all input quantities (model equation) and consequently the uncertainty in $D_{w, Q}$ is determined from the standard uncertainties of all input quantities. The standard uncertainty of a corresponding input quantity is estimated in terms of one standard deviation associated with the assumed probability distribution. The standard uncertainties, denoted by the symbol $u_{i}$, are classified into type A and type B. Type A uncertainties are evaluated on the basis of a statistical analysis of a series of observations. Type B uncertainties are obtained using non-statistical methods. Examples of type B uncertainty contributions are: uncertainties stated in calibration certificates, uncertainties and tolerances stated in specifications given by the manufacturer, uncertainties obtained from literature (reference data). Usually the standard uncertainties for all components are expressed in a relative form. The standard uncertainty in $D_{w, Q}$ is termed combined standard uncertainty and denoted by $u_{c}$, which is determined from all uncertainty contributions of the input quantities $u_{i}$ using the following equation:

$$
u_{c}^{2}=\sum_{i=1}^{N}\left(c_{i} \cdot u_{i}\right)^{2}
$$

where $c_{i}$ are the sensitivity coefficients, defined by:

$$
c_{i}=\partial D_{w, Q} / \partial x_{i}, \text { with } i=1, \ldots, N
$$

Note, that it is assumed that all sources of uncertainties are uncorrelated. Finally, the expanded uncertainty $U$ for $D_{w, Q}$ is calculated by multiplying the combined standard uncertainty $u_{c}$ by a coverage factor $k$, which is mostly chosen $k=2$.

\section{A.7.3 Example of an uncertainty budget for the determination of absorbed dose to water $\mathrm{D}_{\mathrm{w}, \mathrm{Q}}$ in a clinical photon beam $\mathrm{Q}$}

In this example the complete dosimetry system (ionisation chamber and electrometer) has been calibrated at the standards laboratory. Therefore $k_{\text {elec }}=1.00$ and no uncertainty contribution is assumed. Before starting the measurement the dosimeter system was allowed sufficient time to stabilize after switching on and to reach temperature stabilization of the ionisation chamber in the water phantom. In addition the chamber is pre-irradiated to a dose of several gray (2-5 Gy) in order to settle adequately achieving charge equilibrium in the different materials involved. The effect of non-linearity in the response of the dosimeter system is considered to be very small and the resulting uncertainty is neglected. No 
corrections are applied for the chamber orientation in the beam, for any departure of the field size or the radial non-uniformity of the beam and the resulting uncertainties are assumed to be negligible. It is assumed that during the measurements the ambient conditions of temperature, pressure and humidity are constant. As a consequence no uncertainty contributions arise.

\section{A.7.3.1 Notes on the uncertainty contributions of the input quantities}

All relevant uncertainty sources are considered individually and are presented in Table 20.

Explanatory notes concerning the different uncertainty sources are given below:

\section{Uncertainty in calibration certificate}

The expanded uncertainty stated on the calibration certificate from a Primary Standard Dosimetry Laboratory is estimated to be $0.9 \%$ for a coverage factor $k=2$, which corresponds to a confidence level of about $95 \%$ assuming a normal distribution. Usually the calibration coefficient of a dosimeter changes during a time period of several years (typically 2-3 years) between calibrations. The relative change is about $0.3 \%$. Taking this value as a limit and assuming a rectangular distribution a value of $0.173 \%$ for the relative standard uncertainty is determined.

\section{Dose meter reading}

The mean and the standard deviation are obtained from a series of readings (preferably at least ten readings should be taken). The leakage current should be measured before and after the irradiation. Typically the leakage current is less than $0.1 \%$ of the current measured during irradiation and can often be ignored or taken into account in the resulting uncertainty. In this example the dosimeter reading is corrected for the leakage current.

Often the readings are digitized and a resolution uncertainty has to be assigned of \pm 0.5 to the last digit/count on the display assuming a rectangular distribution. In the example a resolution uncertainty of $0.10 \%$ is adopted.

Temperature and pressure correction $k_{T P}$

The correction factor $k_{T P}$ in the air volume of the ionisation chamber is calculated according to equation (17) from the measured values of the temperature $T(\mathrm{~K})$ and the pressure $P$ (hPa). Typical values of relative expanded uncertainties of $0.17 \%(0.5 \mathrm{~K}$ at a temperature of about $293.15 \mathrm{~K}$ ) and $0.10 \%$ are taken from the certificates of the temperature and barometer calibration respectively. 
As the temperature and pressure do not vary during the measurements no type $A$ uncertainties arise. Type B uncertainties arising from the thermometer and barometer resolution result in very small relative standard uncertainties, which can often be neglected in practice. It should be noted that the temperature is not measured in the chamber cavity but close to the ionisation chamber in the water phantom. In this example the difference in temperature between the air cavity and the position of the sensing part of the thermometer in the water phantom is neglected.

\section{Humidity correction $k_{h}$}

In a range between $20 \%-80 \%$ relative humidity the response of an ionisation chamber changes not more than $0.1 \%$. No correction for the humidity effect is made, but an uncertainty contribution assuming a rectangular distribution is assigned to $k_{h}$.

Recombination correction $k_{s}$

Using expression (21) $k_{s}$ is calculated with a relative standard uncertainty of $0.1 \%$.

Polarity correction $k_{p o l}$

For most ionisation chamber types the polarity effect is small and often neglected in photon beams. The relative standard uncertainty in $k_{\text {pol }}$ is typically estimated $0.1 \%$.

Deviation of chamber position (depth) in phantom

The relative change in absorbed dose to water in the vicinity of the reference depth in the water phantom is $0.6 \% / \mathrm{mm}$. The uncertainty in positioning at the reference depth is $0.1 \mathrm{~mm}$ in terms of one standard deviation resulting in a relative standard uncertainty of $0.06 \%$.

\section{Beam quality correction factor $k_{Q}$}

According to section A.4.5.2 of appendix A.4 the relative standard uncertainty in $k_{Q}$ is estimated $0.4 \%$. 
Table 20: Uncertainty budget for the determination of absorbed dose to water $D_{w, Q}$ in a clinical photon beam $Q$

All individual uncertainty contributions are summed in quadrature and from the total sum the root is taken to give the combined standard uncertainty of the result: $u\left(D_{w, Q}\right) / D_{w, Q} \approx 0.8 \%$. The expanded uncertainty is determined by multiplying the combined standard uncertainty with a coverage factor $k=2$.

\begin{tabular}{|c|c|c|c|c|c|c|c|}
\hline $\begin{array}{c}\text { Input quantity } x_{i} \\
\text { Source of uncertainty }\end{array}$ & $\begin{array}{c}\text { Relative } \\
\text { (expanded) } \\
\text { uncertainty }\end{array}$ & $\begin{array}{l}\text { Uncertainty } \\
\text { type }\end{array}$ & $\begin{array}{l}\text { Uncertainty } \\
\text { distribution }\end{array}$ & $\begin{array}{c}\text { Coverage } \\
\text { (reducing) factor }\end{array}$ & $\begin{array}{c}\text { Relative } \\
\text { standard } \\
\text { uncertainty }\end{array}$ & $\begin{array}{l}\text { Sensitivity } \\
\text { coefficient }\end{array}$ & $\begin{array}{l}\text { Uncertainty } \\
\text { contribution }\end{array}$ \\
\hline & $U_{i}$ & & & $k$ & $u_{i}$ & $c_{i}$ & $c_{i} u_{i}$ \\
\hline \multicolumn{8}{|l|}{ Calibration coefficient, $\mathrm{N}_{\mathrm{D}, \mathrm{w}}$} \\
\hline Uncertainty in calibration certificate & $0.90 \%$ & B & normal & 2 & $0.450 \%$ & 1 & $0.450 \%$ \\
\hline Long term stability of calibration coefficient & $0.30 \%$ & $\mathrm{~B}$ & rectangular & 1.73 & $0.173 \%$ & 1 & $0.173 \%$ \\
\hline \multicolumn{8}{|l|}{ Dosimeter reading, corrected for leakage, $\mathrm{M}$} \\
\hline Repeatability & $0.50 \%$ & A & normal & 1 & $0.500 \%$ & 1 & $0.500 \%$ \\
\hline Resolution & $0.10 \%$ & $\mathrm{~B}$ & rectangular & 1.73 & $0.058 \%$ & 1 & $0.058 \%$ \\
\hline \multicolumn{8}{|l|}{ Temperature during measurement, $\mathrm{T}(\mathrm{K})$} \\
\hline Thermometer calibration & $0.34 \%$ & $\mathrm{~B}$ & normal & 2 & $0.170 \%$ & 1 & $0.170 \%$ \\
\hline Thermometer resolution & $0.03 \%$ & B & rectangular & 1.73 & $0.020 \%$ & 1 & $0.020 \%$ \\
\hline \multicolumn{8}{|l|}{ Pressure during measurement, $\mathrm{P}(\mathrm{hPa})$} \\
\hline Barometer calibration & $0.10 \%$ & $\mathrm{~B}$ & normal & 2 & $0.050 \%$ & 1 & $0.050 \%$ \\
\hline Barometer resolution & $0.005 \%$ & B & rectangular & 1.73 & $0.003 \%$ & 1 & $0.003 \%$ \\
\hline \multicolumn{8}{|l|}{ Humidity correction $k_{h}$} \\
\hline Deviation from reference condition of $50 \%$ & $0.10 \%$ & $\mathrm{~B}$ & rectangular & 1.73 & $0.058 \%$ & 1 & $0.058 \%$ \\
\hline \multicolumn{8}{|l|}{ Recombination correction $\boldsymbol{k}_{s}$} \\
\hline Uncertainty in recombination correction factor & $0.10 \%$ & B & normal & 1 & $0.100 \%$ & 1 & $0.100 \%$ \\
\hline \multicolumn{8}{|l|}{ Polarity correction $k_{\text {pol }}$} \\
\hline Uncertainty in polarity correction factor & $0.10 \%$ & B & normal & 1 & $0.100 \%$ & 1 & $0.100 \%$ \\
\hline \multicolumn{8}{|l|}{ Depth positioning ionisation chamber in phantom } \\
\hline Uncertainty due to deviation from ref. position & $0.12 \%$ & B & normal & 2 & $0.060 \%$ & 1 & $0.060 \%$ \\
\hline \multicolumn{8}{|l|}{ Beam quality correction factor $k_{Q}$} \\
\hline Uncertainty in the quality correction factor & $0.8 \%$ & $\mathrm{~B}$ & normal & 2 & $0.400 \%$ & 1 & $0.400 \%$ \\
\hline
\end{tabular}




\section{References}

[1] NCS, Netherlands Commission on Radiation Dosimetry. Report 2: Code of practice for the dosimetry of high energy photon beams. Delft, The Netherlands, 1986.

[2] NCS, Netherlands Commission on Radiation Dosimetry. Report 5: Code of practice for the dosimetry of high energy electron beams. Delft, The Netherlands, 1989.

[3] Mijnheer B.J., Wittkamper F.W., Comparison of recent codes of practice for high energy photon dosimetry, Phys. Med. Biol. 31 407-16, 1986.

[4] AAPM TG-21, A protocol for the determination of absorbed dose from high energy photon and electron beams, Med. Phys. 10 741-771, 1983.

[5] IAEA, Absorbed dose determination in photon and electron beams: an international code of practice Technical Report Series 277 (Vienna: IAEA), 1987.

[6] Hoornaert M.T., Van Dam J., Vynckier S., Bouiller A. A dosimetric quality audit of photon beams by the Belgian hospital physicists association. Radiother.Oncol. 28 37-43, 1993.

[7] Wittkamper F.W., Mijnheer B.J., van Kleffens H.J., Dose intercomparison at the radiotherapy centers in The Netherlands. Part I. Photon beams under reference conditions and for prostatic cancer treatment, Radiother. Oncol. 9 33-44, 1987.

[8] Bielajew A.F., Rogers D.W.O., Implications of new correction factors on primary air kerma standards in 60Co beams, Phys. Med. Biol. 37 1283-1291, 1992.

[9] Almond P.R., Biggs P.J., Coursey B.M., Hanson W.F., Saiful Huq M., Nath R. and Rogers D.W.O., AAPM's TG-51 protocol for clinical reference dosimetry of high energy photon and electron beams, Med. Phys. 26 1847-1870, 1999.

[10] IAEA, Absorbed dose determination in external beam radiotherapy: an international code of practice for dosimetry based on standards of absorbed dose to water, Technical Report Series 398 (Vienna: IAEA), 2000.

[11] Wittkämper F.W., Mijnheer B.J., Dose intercomparison at the radiotherapy centers in The Netherlands, Part III: Characteristics of electron beams, Radiother. Oncol. 27 156-163, 1993.

[12] Huq M.S., Hossain M. and Andreo P. A., Comparison of the AAPM TG-51 protocol and the IAEA absorbed-dose to water based Code of Practice for dosimetry calibration of high energy photon beams, Med. Phys. 26 1153, 1999.

[13] Ding G.X., Cygler J.E. and Kwok C. B., Clinical reference dosimetry: comparison between AAPM TG-21 and TG-51 protocols, Med. Phys. 27 1217-1225, 2000. 
[14] Shortt K.R., Shobe J. and Domen S., Comparison of dosimetry calibration factors at the NRCC and the NIST, Med. Phys. 27 1644-1654, 2000.

[15] Huq M.S. and Andreo P., Reference dosimetry in clinical high energy photon beams: comparison of the AAPM TG-51 and AAPM TG-21 dosimetry protocols, Med. Phys. 28 46-54, 2001.

[16] Palmans H., Nafaa L., De Jans J., Gillis S., Hoornaert M.T., Martens C., Piessens M., Thierens H., Van der Plaetsen A., Vynckier S., Absorbed dose to water based dosimetry versus air kerma based dosimetry for high energy photon beams: an experimental study, Phys. Med. Biol. 47 421-40, 2002.

[17] Palmans H., Nafaa L., de Patoul N., Denis J-M., Tomsej M. and Vynckier S., A dosimetry study comparing NCS report-5, IAEA TRS-381, AAPM TG-51 and IAEA TRS-398 in three clinical electron beam energies, Phys. Med. Biol. 48 1091-1107, 2003.

[18] Hohlfeld K., The standard DIN 6800: Procedures for absorbed dose determination in radiology by the ionisation method (IAEA-SM-298/31), Dosimetry in Radiotherapy Vol I (Vienna: IAEA) 13-22,1988.

[19] Andreo P., Absorbed dose beam quality factors for the dosimetry of high energy photon beams, Phys. Med. Biol. 37 2189-2211, 1992.

[20] Rogers D.W.O., The advantages of absorbed dose calibration factors, Med. Phys. 19 1227-1239, 1992.

[21] Domen S.R., "A Sealed Water Calorimeter for Measuring Absorbed Dose," J. Res. Natl. Inst. Stand. Technol. 99 121-141, 1994.

[22] ICRU, Stopping Powers for Electrons and Positrons, Report 37 (Bethesda,MD: ICRU), 1984.

[23] ICRU, Tissue Substitutes in Radiation Dosimetry and Measurements, Report 44 (Bethesda,MD:ICRU), 1989.

[24] White D.R., Tissue substitutes in experimental radiation physics, Med. Phys. 5 467-479, 1978.

[25] Tello, V.M., Tailor R.C., and Hanson W.F., How water equivalent are waterequivalent solid materials for output calibration of photon and electron beams?, Med. Phys. 22 1177-1189, 1995.

[26] Brahme A., Svensson H., Radiation beam characteristics of $22 \mathrm{MeV}$ Microtron, Acta Radiol. Oncol. 18 244-272, 1979. 
[27] Greening J.R., Fundamentals of Radiation Dosimetry, Medical Physics Handbooks, Adam Hilger (Bristol), 1981.

[28] Brahme A., Andreo P., Dosimetry and quality specification of high energy photon beams, Acta Radiol.Oncol. 25 213-223, 1986.

[29] ICRU, Dosimetry of High Energy Photon Beams based on Standards of Absorbed Dose to Water, Report 64 (Bethesda, MD:ICRU), 2001.

[30] IAEA, The use of plane-parallel ionisation chambers in high energy electron and photon beams. An international code of practice for dosimetry, Technical Report Series 381 (Vienna: IAEA), 1997.

[31] Burns D.T., Ding G.X. and Rogers D.W.O., $R_{50}$ as a beam quality specifier for selecting stopping power ratios and reference depths for electron dosimetry, Med. Phys. 23 383-388, 1996.

[32] Ding G.X., Rogers D.W.O., Mackie T.R., Calculation of stopping-power ratios using realistic clinical electron beams, Med. Phys. 22 489-501, 1995.

[33] Parkkinen R., Kosunen A., Sipilä P., Järvinen H., Development of calibration procedures for the electron beam calibration of plane-parallel ionisation chambers, Standards and Codes of Practice in Medical Radiation Dosimetry, Vol.1 (Proc. Symp. Vienna, 2002) 361-366, 2003.

[34] CCEMRI(I), Correction d'Humidité, CCEMRI(I) R(I)-30, BIPM (Sèvres), 1977.

[35] Boag J.W., The Dosimetry of lonising Radiation, Ed. Kase K.R., Bjarngard B.E., Attix F.H., Vol. 2 169-243, Academic Press (New York), 1987.

[36] Boag J.W., Currant J., Current collection and ionic recombination in small cylindrical ionisation chambers exposed to pulsed radiation, Br. J. Radiol. 53471 478, 1980.

[37] Boutillon M, Volume recombination parameter in ionisation chambers, Phys. Med. Biol. 43 2061-2072,1998.

[38] Boag J.W., Ionisation measurements at very high intensities. 1. Pulsed radiation beams, Br. J. Radiol. 23 601-611, 1950.

[39] ICRU, The Dosimetry of Pulsed Radiation, Report 34 (Bethesda, MD: ICRU), 1982.

[40] Weinhous M.S., Meli A.M., Determinating $P_{\text {ion, }}$ the correction factor for recombination losses in an ionisation chamber, Med. Phys. 11 846-849, 1984.

[41] Institute of Physical Sciences in Medicine (IPSM), Code of practice for high energy photon therapy beams based on the NPL absorbed dose calibration service, Phys. Med. Biol. 35 1355-1360, 1990. 
[42] Burns D.T., McEwen M.R., Ion recombination corrections for the NACP parallel plate chamber in a pulsed electron beam, Phys. Med. Biol. 43 2033-2045, 1998.

[43] Derikum K., Roos M., Measurement of saturation correction factors of thimble type ionisation chambers in pulsed photon beams, Phys. Med. Biol. 38 755-763, 1993.

[44] Burns J.E., Burns D.T., Comments on ion recombination corrections for planeparallel and thimble chambers in electron and photon radiation, Phys. Med. Biol. 38 1986-1988, 1993.

[45] Pieksma M.W.H., de Prez L.A. and Aalbers A.H.L., The NMi water calorimeter, Proceedings of the International Workshop on Recent Developments in Accurate Radiation Dosimetry (McGill University, Montreal), AAPM Proceedings Series 13, Medical Physics Publishing, Madison, Wisconsin, 108-119, 2002.

[46] Pieksma M.W.H., de Prez L.A., van Dijk E., Aalbers A.H.L., Measurements of $k_{Q}$ beam quality correction factors for the NE 2611A chamber in high energy photon beams using the NMi water calorimeter, Standards and Codes of Practice in Medical Radiation Dosimetry, Vol.1 (Proc. Symp. Vienna, 2002) 93-102, 2003.

[47] Palmans H. and Seuntjens J., Construction, Correction Factors and Relative Heat Defect of a High Purity, $4{ }^{\circ} \mathrm{C}$ Water Calorimeter for Absorbed Dose Determinations in High Energy Photon Beams, Proceedings of the NPL Calorimetry Workshop, (Teddington,12-14 October 1994), 1995.

[48] Seuntjens J. and Palmans H., Correction factors and performance of a $4^{\circ} \mathrm{C}$ sealed water calorimeter, Phys. Med. Biol., 44 627-646, 1999

[49] Palmans H., Water calorimetry as primary standard dosimetry method for clinical high energy photon, and proton beams PhD Thesis, Ghent University, Belgium (in Dutch), 1999.

[50] Palmans H., Experimental verification of simulated excess heat effects in the sealed water calorimeter, Recent Advances in Calorimetric Absorbed Dose Standards (Proc. Workshop, Teddington1999), NPL report CIRM 42, 74-84, 2000.

[51] Palm A., Mattson O., Experimental determination of beam quality conversion factors $\mathrm{k}(\mathrm{Q})$ in clinical photon beams using ferrous sulphate (Fricke) dosimetry, Med. Phys. 29 2756-2762, 2002.

[52] Medin J., Ross C.K., Klassen N.V., Palmans H., Grusell E. and Grindborg J-E., Experimental determination of beam quality $\mathrm{k}_{\mathrm{Q}, \mathrm{Q}_{0}}$ factors for two types of Farmer chambers in a $10 \mathrm{MV}$ photon and a $175 \mathrm{MeV}$ proton beam, Phys. Med. Biol, accepted, 2006. 
[53] Palmans H., Mondelaers W., Thierens H., Absorbed dose beam quality correction factors $\mathrm{k}_{\mathrm{Q}}$ for the NE 2571 chamber in a $5 \mathrm{MV}$ and a $10 \mathrm{MV}$ photon beam, Phys. Med. Biol. 44 647-663, 1999.

[54] Ross C.K., Shortt K.R., Rogers D.W.O. and Delaunay F., A test of $\operatorname{TPR}_{20,10}$ as a beam quality specifier for high energy photon beams, Measurement Assurance in Dosimetry (Proc. Symp. Vienna, 1993) IAEA, 309-321,1994.

[55] Seuntjens J.P., Ross C.K., Shortt K.R., Rogers D.W.O., Absorbed-dose beam quality conversion factors for cylindrical chambers in high energy photon beams, Med. Phys. 27 2763-2779, 2000.

[56] Shortt K.R., Ross C.K., Schneider M., Hohlfeld K., Roos M., Perroche A.M., A comparison of absorbed dose standards for high energy X-rays, Phys. Med. Biol. 38 1937-1955, 1993.

[57] Boutillon M., Coursey B.M., Hohlfeld K., Owen B. and Rogers D.W.O., Comparison of primary water absorbed dose standards, Measurement Assurance in Dosimetry (Proc. Symp. Vienna, 1993) IAEA, 95-111,1994.

[58] Guerra A.S., Laitano R.F., Pimpinella M., Experimental determination of the beam quality dependence factors, $\mathrm{k}_{\mathrm{Q}}$, for ionisation chambers used in photon and electron dosimetry, Phys. Med. Biol. 40 1177-1190, 1995.

[59] Palmans H., Mondelaers W., Thierens H., Beam quality of high energy photon beams at the Ghent University linear accelerator, Phys. Med. Biol. 47 L15-18, 2002.

[60] Andreo P., A comparison between calculated and experimental $\mathrm{kQ}$ photon beam quality correction factors, Phys. Med. Biol. 45 L25-L38, 2000.

[61] Ross C.K., private communication, 2005.

[62] Duane S. and Simon M.H., Experimental determination of the quality-dependent correction factor $k_{Q}$ for ionisation chambers of type NE2561, NE2611 and NE2571, (Proceedings of the World Congress on Medical Physics and Biomedical Engineering, Aug 24-29, 2003, Sydney, Australia) [CD-ROM] ISBN 1877040142 , Paper No. 4433, 2003.

[63] Stücki G., Schafer R., Quintel H., The METAS photon-beam primary standard sealed water calorimeter, AbsDos 2003, workshop on Recent Advances in Absorbed Dose Standards (Proc. http://www.arpansa.gov.au/absdos/proc.htm Australian Radiation Protection and Nuclear Safety Agency, 19-21 August 2003, Yallambie, Victoria), 2003. 
[64] McEwen M., private communication, 2006.

[65] EUROMET 605, LNHB (2006), to be published.

[66] Allisy-Roberts P.J., Burns D.T., Palmans H., Comparison of the standards of absorbed dose to water of the LSDG, Belgium and the BIPM for 60Co $\mathrm{Y}$-rays, Rapport BIPM 2000/01.

[67] Kosunen A., Rogers D.W.O., Beam quality specification for photon beam dosimetry, Med. Phys. 20 1181-1188, 1993.

[68] Li X.A., Rogers D.W.O., Reducing electron contamination at dose maximum in photon beams, Med. Phys. 21 791-798, 1994.

[69] Rogers D.W.O., Correcting for electron contamination at dose maximum in photon beams, Med. Phys. 26 533-537, 1999.

[70] Levenberg K., A Method for the solution of certain non-linear problems in least squares, Quart. Appl. Math. 2 164-168, 1944.

[71] Marquardt D., An algorithm for least-squares estimation of non-linear parameters, J. Soc. Industr. \& Appl. Math. 11 431-441, 1963.

[72] Krauss A., and Kapsch R.P., Calorimetric determination of $k_{Q}$ factors for NE 2561 and NE 2571 ionization chambers in $5 \mathrm{~cm} \times 5 \mathrm{~cm}$ and $10 \mathrm{~cm} \times 10 \mathrm{~cm}$ radiotherapy beams of 8 MV and 16 MV photons, Phys. Med. Biol. 52 6243-6259, 2007.

[73] Andreo P., Improved calculations of stopping power ratios and their correlation with the quality of therapeutic photon beams, Measurement Assurance in Dosimetry (Proc. Symp. Vienna, 1993) IAEA, 335-359, 1994.

[74] Rogers D.W.O., Accuracy of the Burns equation for stopping-power ratio as a function of depth and R for , Med. Phys. 31 2961-2963, 2004.

[75] Stewart K., Seuntjens J., Ross C. and Klassen N., Water calorimeter-based beam quality conversion factors for clinical electron beams, Radiother. Oncol. 76 (suppl. 2), p. S76, 2005.

[76] Verhaegen F., Zakikhani R., DuSautoy A., Palmans H., Bostock H., Shipley D. and Seuntjens J., Perturbation correction factors for the NACP-02 plane-parallel ionisation chamber in water in high energy electron beams, Phys. Med. Biol. 51 1221-1235, 2006.

[77] Buckley L.A. and Rogers D.W.O., Wall correction factors, $P_{\text {wall }}$, for parallel-plate ionisation chambers, Med. Phys. 33 1788-1796, 2006. 
[78] McEwen M., Palmans $H$. and Williams A., An empirical method for the determination of wall perturbation factors for parallel-plate chambers in high energy electron beams, Phys. Med. Biol. 51 5167-5181, 2006.

[79] Ma C-M. and Nahum A.E., Plane parallel chambers in electron beams: Monte Carlo findings on the perturbation factor, Measurement Assurance in Dosimetry (Proc. Symp. Vienna, 1993) IAEA, 481-493,1994.

[80] Sempau J., Andreo P., Aldana J., Mazurier J. and Salvat F., Electron beam quality correction factors for plane-parallel ionisation chambers: Monte Carlo calculations using the PENELOPE system, Phys. Med. Biol. 49 4427-4444, 2004.

[81] Ding G.X. and Cygler J.E., Measurement of $P_{\text {repl }} P_{\text {wall }}$ factors in electron beams and in a ${ }^{60} \mathrm{Co}$ beam for plane-parallel chambers, Med. Phys. 25 1453-1478, 1998.

[82] Palm A., Mattsson O. and Andreo P., Calibration of plane-parallel chambers and determination of $\mathrm{p}_{\text {wall }}$ for the NACP and Roos chambers for ${ }^{60}$ Co-ray beams, Phys. Med. Biol. 45 971-981,2000.

[83] Dohm O.S., Christ G., Nusslin F., Electron dosimetry based on the absorbed dose to water concept: A comparison of the AAPM TG-51 and DIN 6800-2 protocols, Med. Phys. 28 2258-2264, 2001.

[84] Palm Å., Czap L., Andreo P., and Mattsson O., Performance analysis and determination of the $p_{\text {wall }}$ correction factor for 60Co gamma-ray beams for Wellhöfer Roos-type plane-parallel chambers, Phys. Med. Biol. 47 631-640, 2002.

[85] Stewart K.J. and Seuntjens J.P., Comparing calibration methods of electron beams using plane-parallel chambers with absorbed dose to water based protocols, Med. Phys. 29 284-289, 2002.

[86] Christ G., Dohm O.S., Bruggmoser G. and Schüle E., The use of plane-parallel chambers in electron dosimetry without any cross-calibration, Phys Med Biol 47 N121- N126, 2002.

[87] Johansson K.A., Mattson L.O., Lindborg L., Svensson H., Absorbed-dose determination with ionisation chambers in electron and photon beams having energies between 1 and $50 \mathrm{MeV}$, National and International Standardization of Radiation Dosimetry (Proc. Symp. Atlanta, 1977), Vol. 2, IAEA, 243-270, 1978.

[88] Ma C.M., Nahum A.E., Effect of the size and composition of the central electrode on the response of cylindrical ionisation chambers in high-energy photon and electron beams, Phys. Med. Biol. 38 267-290, 1993. 
[89] Kapsch R.P., Bruggmoser G., Christ G., Dohm O.S., Hartmann G.H. and Schüle E., Experimental determination of $p_{\mathrm{Co}}$ perturbation factors for plane-parallel chambers, Phys. Med. Biol. 52 7167-7181, 2007.

[90] DIN, Dosismessverfahren nach der Sondenmethode für Photonen- und Elektronenstrahlung Teil 2: Ionisationsdosimetrie DIN 6800-2 (Berlin: Deutsches Institut für Normung), 1997.

[91] Mainegra-Hing E., Kawrakow I., Rogers D.W.O., Calculations for plane-parallel ion chambers in 60Co beams using the EGSnrc Monte Carlo code, Med Phys. 30 179189, 2003.

[92] Ross C.K. and Shortt K.R., The effect of waterproofing sleeves on ionisation chamber response, Phys. Med. Biol. 37 1403-1411, 1992

[93] Guide to the Expression of Uncertainty in Measurement, ISO, Geneva, $2^{\text {nd }}$ Ed., 1995.

[94] EA-4/02, Expression of uncertainty of measurement in calibration, European Cooperation for Accreditation, 1999. 


\section{Acknowledgements}

The members of the subcommittee would like to thank Prof. Dr. Jan van Dam (Emeritus) of Katholieke Universiteit Leuven (Belgium) and Dr. Jan Seuntjens of McGill University in Montreal (Canada) for their detailed reviews of the Code of practice and their valuable comments and suggestions. We also wish to thank the staff of the lonising Radiation Section of NMi and the medical physics staff of the radiotherapy institutes and hospital departments participating in the $k_{Q}$ measurements in selected clinical photon beams, conducted in Belgium and the Netherlands. We also gratefully acknowledge the support of Stratec Services B.V., Houten (The Netherlands) and PI Medical Diagnostic Equiment B.V.,Tijnje (The Netherlands) for the loan of ionisation chambers. Sets of cylindrical ionisation chambers (Wellhöfer FC65-G and NE 2571 and NE2611) and plane-parallel ionisation chambers from Wellhöfer were provided by Stratec Services B.V. and PTW 30012 cylindrical ionisation chambers were provided by PI Medical Diagnostic Equipment B.V.. 


\title{
Publications of the Netherlands Commission on Radiation Dosimetry
}

\author{
Radiation dosimetry activities in the Netherlands. \\ Inventory compiled under the auspices of the Netherlands Commission for Radiation Dosimetry. \\ NCS Report 1, July 1986.
}

Code of practice for the dosimetry of high-energy photon beams.

NCS Report 2, December 1986.

Proceedings of the Symposium on Thermoluminescence Dosimetry.

NCS Report 3, October 1988.

\author{
Aanbevelingen voor dosimetrie en kwaliteitscontrole van radioactieve bronnen bij \\ brachytherapie \\ NCS Report 4, Februari 1989 (in Dutch). \\ Recommendations for dosimetry and quality control of radioactive sources used in \\ brachytherapy. \\ Synopsis (in English) of NCS Report 4, February 1991. \\ Code of practice for the dosimetry of high-energy electron beams. \\ NCS Report 5, December 1989. \\ Dosimetric aspects of Mammography. \\ NCS Report 6, March 1993.
}

Recommendations for the calibration of Iridium-192 high dose rate sources.

NCS Report 7, December 1994.

Kwaliteitscontrole van Medische Lineaire Versnellers, methoden voor kwaliteitscontrole, wenselijke toleranties en frequenties.

NCS Report 8, December 1995 (in Dutch).

Quality Control of Medical Linear Accelerators, current practice and minimum requirements. NCS Report 9, August 1996.

Dosimetry of low and medium energy $x$-rays, a code of practice for use in radiotherapy and radiobiology.

NCS Report 10, July 1997.

Quality Control of Simulators and CT scanners and some basic QC methods for Treatment Planning Systems, current practice and minimum requirements.

NCS Report 11, September 1997.

Determination and use of scatter correction factors of megavoltage photon beams, measurement and use of collimator and phantom scatter correction factors of arbitrarily shaped fields with a symmetrical collimator setting.

NCS Report 12, March 1998. 
Quality Assurance in Brachytherapy.

NCS Report 13, October 2000.

Quality control of sealed beta sources in brachytherapy.

NCS Report 14, August 2004.

Quality assurance of 3-D treatment planning systems for external photon and electron beams NCS Report 15, January 2006

Monte Carlo Treatment Planning, An Introduction.

NCS Report 16, June 2006

Dosimetrie in de Radiologie: Stralingsbelasting van de Patiënt en Werknemers

NCS Rapport 17, Maart 2007 (in Dutch)

Code of Practice for the Absorbed Dose Determination in High Energy Photon and Electron Beams

NCS Report 18, January 2008 
From outside The Netherlands, please complete the order form below.

\section{ORDER FORM}

Please send this completed order form to:

NCS secretary, P.O.Box 654, 2600 AR DELFT, The Netherlands

QTY REPORT TITLE

Subtotal

Add postage and packing (Europe $€ 10,-$, outside Europe $€ 15,-$ )

Total

Payment by: $\quad \Upsilon \quad$ Cheque/postal order made payable to Netherlands Commission on Radiation Dosimetry (add $€ 7,50$ for banking costs)

$\Upsilon \quad$ Please charge my Eurocard/Mastercard

Credit card No.

Expiry date

Name:

Address:

Date: Signature:

" please delete as applicable

For more information, see: http://www.radiationdosimetry.org/ 